\title{
Pneumococcal Colonization and Carriage
}

\author{
Invited Lecture Abstracts
}

\author{
ISPPD-0534 \\ Pneumococcal Colonization and Carriage
}

\section{GENOMICS AND PNEUMOCOCCAL EPIDEMIOLOGY IN THE NASOPHARYNX}

C. Chewapreecha ${ }^{1}$, S.R. Harris ${ }^{1}$, N.J. Croucher ${ }^{2}$, C. Turner ${ }^{3}$, P. Marttinen ${ }^{4}$, L. Cheng ${ }^{5}$, A. Pessia 5 , D. Aanensen ${ }^{6}$, S.J. Salt$\mathrm{er}^{1}$, A.E. Mather ${ }^{1}$, A.J. Page ${ }^{1}$, D. Harris ${ }^{1}$, F. Nosten ${ }^{7}$, D. Goldblatt ${ }^{8}$, J. Corander ${ }^{5}$, J. Parkhill ${ }^{1}$, P. Turner ${ }^{3}$, S.D. Bentley ${ }^{1}$

${ }^{1}$ Pathogen genomics, The Wellcome Trust Sanger Institute, Cambridge, United Kingdom; ${ }^{2}$ Center for Communicable Disease Dynamics, Harvard School of Public Health, Boston, USA; ${ }^{3}$ Cambodia-Oxford Medical Research Unit, Angkor Hospital for Children, Siem Reap, Cambodia ${ }^{4}$ Department of Information and Computer Science, Aalto University, Helsinki, Finland; ${ }^{5}$ Department of Mathematics and Statistics, University of Helsinki, Helsinki, Finland; ${ }^{6}$ Department of Infectious Disease Epidemiology, Imperial College London, London, United Kingdom; ${ }^{7}$ Shoklo Malaria Research Unit, Mahidol-Oxford Tropical Medicine Research Unit, Tak, Thailand; ${ }^{8}$ Immunobiology Unit, Institute of Child Health University College London, London, United Kingdom

Homologous recombination is one of the main evolutionary forces affecting Streptococcus pneumoniae. The highly recombinogenic nature of this species allows introduction of genetic material with selective advantages in carriage, the state that is a prerequisite for the development of pneumococcal invasive diseases. To study the impact of recombination on the evolution of a carriage pneumococcal population, whole genome sequencing was used to characterize 3,085 pneumococcal carriage isolates from a $2.4 \mathrm{~km}^{2}$ Thai refugee camp collected over a 3-year period. This high sampling density allowed us to characterized genetic exchanges in the pneumococcal population at a high resolution. Recombination 'hotspots' showed remarkable consistency between lineages, some of which were associated with drug resistance. Temporal trends in recombination at these sites reflected changes in antibiotic consumption, suggesting recombination facilitates adaptation to changing selection pressures. The highest frequencies of receipt and donation of DNA fragments exchanged through homologous recombination were observed in non-encapsulated lineages, implying a potential role in diversification and adaptation of the overall population played by these non-vaccine target lineages. These findings expand our understanding of pneumococcal population in carriage and help inform the design of future intervention strategies.

No conflict of interest

ISPPD-0550

Pneumococcal Colonization and Carriage

\section{INTERACTIONS BETWEEN STREPTOCOCCUS PNEUMONIAE AND THE RESPIRATORY MICROBIOME IN RELATION TO RESPIRATORY HEALTH}

\section{Bogaert ${ }^{1}$ \\ ${ }^{1}$ The Netherlands}

Individuals differ markedly in their susceptibility to and clinical presentation of respiratory infections, despite that most young children and many adults are colonized with potential bacterial pathogens like Streptococcus pneumoniae. The reasons for these individual differences are not yet fully understood, but clearly multifactorial. Besides pathogen-related (virulence)-factors, host-related factors like immune-status and genetic background and environmental factors, a possible fourth factor might be of relevance, i.e. the commensal community of bacteria 'hosting' these potential pathogens. The collective genomes of these commensal inhabitants are referred to as the human microbiome. This microbiome contains highly complexity communities of bacteria, which differ between individuals and even more between niches. The human microbiome in general has shown crucial for an appropriate development of our immune system and our mucosal barriers, and for prevention of pathogen adherence and expansion. We studied the development and composition of microbiota of the upper respiratory tract in different age-groups and in relation to environmental and disease characteristics. We observed highly complex and nichespecific communities of bacteria. Even within the upper respiratory tract, the microbiota composition differs depending on the exact anatomical location. Furthermore, the composition of respiratory microbiota varies with host and environmental factors like age, season, infant feeding, and viral presence. Moreover, our data suggest a correlation between the presence and abundance of specific bacteria and stability of microbiota, as well as susceptibility to respiratory infections. Finally, clear evidence has been found for patterns of bacterial interactions within the respiratory microbiota, with a central role played by Streptococci.

No conflict of interest 


\title{
Oral Poster Abstracts
}

ISPPD-0085

Pneumococcal Colonization and Carriage

\section{THE ADULT NASOPHARYNGEAL MICROBIOME AS A DETERMINANT OF PNEUMOCOCCAL CARRIAGE}

A.J.H. Cremers' ${ }^{1}$ A.L. Zomer' ${ }^{1}$, J.F. Gritzfeld ${ }^{2}$, J.G. Ferwerda ${ }^{1}$, S.A.F.T. van Hijum³, M.I. de Jonge ${ }^{1}$, K.P. Klugman ${ }^{4}$, J. Boekhorst $^{3}$, H.M. Timmerman ${ }^{5}$, S.B. Gordon ${ }^{2}$, P.W.M. Hermans ${ }^{1}$

${ }^{1}$ Dept of Pediatrics, Radboud University Medical Centre, Nijmegen, Netherlands; ${ }^{2}$ Dept of Clinical Sciences, Liverpool School of Tropical Medicine, Liverpool, United Kingdom; ${ }^{3}$ Centre for Molecular and Biomolecular Informatics (CMBI) Bacterial Genomics, Radboud University Medical Centre, Nijmegen, Netherlands; ${ }^{4}$ The Rollins School of Public Health, Emory University, Atlanta, USA; ${ }^{5}$ Dept of Health, NIZO food research B.V., Ede, Netherlands

Although cohort studies have indicated associations between Streptococcus pneumoniae and other microbes in the nasopharynx, they do not allow statements on causality. Here a unique experimental human pneumococcal carriage model is used to study whether the nasopharyngeal microbiome is perturbed by pneumococcal exposure and whether a specific composition determines subsequent establishment of pneumococcal carriage. Healthy adult volunteers were assessed for pneumococcal carriage by culture of nasal wash samples (NWS). Those without naturally acquired pneumococcal carriage received an experimental intranasal pneumococcal challenge with serotype $6 \mathrm{~B}$ or $23 \mathrm{~F}$. The composition of the nasopharyngeal microbiome was longitudinally studied by $16 \mathrm{~S}$ rDNA pyrosequencing on NWS collected before and 2, 7 and 14 days after challenge. Among the 40 selected volunteers 10 were natural carriers and 30 were experimentally challenged. Microbiome composition data were attained for 117 NWS. By principal component analysis of the individual nasopharyngeal communities before challenge we identified 5 distinct microbiome profiles. Natural pneumococcal carriage was particularly common in one of these profiles $(p=0.005)$. A more diverse microbiome $(p=0.034)$ and low presence of Corynebacterium spp. ( $p=0.046)$ prior to challenge were associated with establishment of pneumococcal carriage. Whereas the nasopharyngeal microbiome was not perturbed after exposure to serotype 6B, its diversity increased upon exposure to serotype $23 \mathrm{~F}(p=0.004)$. Five nasopharyngeal microbiome profiles were identified with different natural pneumococcal carriage rates. A more diverse microbiome and low presence of Corynebacterium spp. prior to challenge were associated with establishment of pneumococcal carriage. Perturbation of the nasopharyngeal microbiome upon pneumococcal exposure seems to be strain dependent.

No conflict of interest

ISPPD-0060

Pneumococcal Colonization and Carriage

\section{MACROPHAGE MIGRATION INHIBITORY FACTOR (MIF) IS IMPORTANT FOR CLEARANCE OF PNEUMOCOCCAL COLONIZATION}

\author{
R. Das ${ }^{1}$, M. LaRose ${ }^{1}$, R. Bucala², J.N. Weiser ${ }^{1}$ \\ ${ }^{1}$ Internal Medicine, University of Pennsylvania School of Medicine, Philadelphia, USA; ${ }^{2}$ Internal Medicine, Yale School of Medicine, New Haven, \\ USA
}

Human genetic polymorphisms associated with reduced expression of macrophage migration inhibitory factor (MIF) have been linked to the risk of community-acquired pneumonia (CAP). Since Streptococcus pneumoniae is a leading cause of CAP and nasal carriage is a precursor to invasive disease, we explored the role of MIF in pneumococcal colonization using a mouse model. Pneumococcal colonization led to local and systemic MIF production. Mice deficient in MIF (Mif ${ }^{\prime-}$ ) were prone to higher density and more prolonged colonization compared to wild-type. The delayed clearance in Mif/- mice correlated with reduced recruitment of nasopharyngeal macrophages. The upregulation and positive feedback provided by monocyte chemotactic protein-1 (MCP-1 or CCL2) was impaired in the $\mathrm{Mif}^{\prime-}$ mice. In vitro, pneumococcal infection of macrophages induced transcription and release of MIF, processes dependent on bacterial expression of the pore-forming toxin, pneumolysin, and induction of MAP kinase phosphorylation. A point mutation in pneumolysin, which eliminates pore-formation, was sufficient to abrogate macrophage production of MIF. Correspondingly, nasal colonization with pneumolysin-deficient bacteria led to decreased local MIF upregulation and reduced macrophage recruitment. Prior work has demonstrated that pneumolysin-deficient bacteria show delayed clearance of colonization; this effect was eliminated in Mif $^{\prime-}$ animals. Downstream of primary clearance, Mif/- animals also demonstrated reduced anti-pneumococcal antibody production and reduced ability to clear secondary colonization. Finally, delivery of MIF to the nasopharynx restored macrophage recruitment and pneumococcal clearance. Our work suggests that MIF is important for innate and adaptive immunity to pneumococcal colonization and could be a contributing factor in the genetic susceptibility to CAP.

No conflict of interest 
ISPPD-0093

Pneumococcal Colonization and Carriage

\section{MULTIPLE SEROTYPE COLONIZATION OF STREPTOCOCCUS PNEUMONIAE IS ASSOCIATED WITH ACUTE RESPIRATORY INFECTIONS IN CHILDREN}

B.G. Dhoubhadel ${ }^{1}$, L. Yoshida ${ }^{2}$, H.A. Nguyen ${ }^{3}$, L.N. Minh ${ }^{1}$, H.T. Le ${ }^{4}$, M. Yasunami ${ }^{1}$, M. Suzuki ${ }^{1}$, K. Morimoto ${ }^{1}$, D.A. Dang ${ }^{3}$, K. Ariyoshi ${ }^{1}$

${ }^{1}$ Department of Clinical Medicine, Institute of Tropical Medicine Nagasaki University, Nagasaki, Japan; ${ }^{2}$ Department of Pediatric Infectious Diseases, Institute of Tropical Medicine Nagasaki University, Nagasaki, Japan; ${ }^{3}$ Department of Bacteriology, National Institute of Hygiene and Epidemiology, Hanoi, Vietnam; ${ }^{4}$ Khanh Hoa Health Service Centre, Khanh Hoa Provincial Health Department, NhaTrang, Vietnam

Background and Aims: Although prevalence of multiple serotype colonization is reported, its association with a clinical outcome and inter-relationship among the serotypes are not known. By applying the newly developed assay based on nanofluidic real time PCR system, we aimed to examine the association of multiple serotype colonization with acute respiratory infections (ARI) and the inter-relationship of the serotypes in terms of bacterial load.

Methods: Nasopharyngeal samples from 595 hospital admitted ARI cases and 350 healthy controls from Nha Trang, Veitnam were screened for Streptococcus pneumoniae by culture and lytA PCR. Samples positive for culture and lytA were subjected to the nanofluidic real time PCR system that can identify 50 serotypes in 29 groups, and it can detect minor population in the multiple serotype colonization, with the minimum level of detection of 30 to 300 copies. Results: The prevalence of multiple serotype colonization was 2.5 times higher in cases than controls, $18.5 \%$ versus $7.1 \%$ (odd ratio $(\mathrm{OR}) 2.96,95 \% \mathrm{Cl}: 1.37-6.93, p=0.002$ ). Among serotypes of multiple serotype colonization, one serotype dominated over the other serotypes by contributing more than $99 \%$ of the total pneumococcal load ( $p<$ $0.001)$. Serotypes covered by conjugate vaccines: PCV7, PCV10 and PCV13 were similar, $75 \%$ in cases and $52 \%$ in controls.

Conclusion: Multiple serotype colonization is associated with ARI cases; one serotype that is dominant over the other, seems to be the causal pathogen. Introduction of PCV will reduce the burden of ARI cases in Vietnam.

No conflict of interest

ISPPD-0502

Pneumococcal Colonization and Carriage

\section{MECHANISMS OF PNEUMOCOCCAL TRANSMISSION: COLONISATION IS ASSOCIATED WITH RHINITIS IN HEALTHY PRESCHOOL CHILDREN}

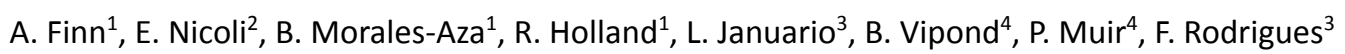

${ }^{1}$ Cellular and Molecular Medicine, University of Bristol, Bristol, United Kingdom; ${ }^{2}$ Social and Community Medicine, University of Bristol, Bristol, United Kingdom; ${ }^{3}$ Infectious Diseases Unit \& Emergency Service, Hospital Pediatrico de Coimbra, Coimbra, Portugal; ${ }^{4}$ South West Regional Virology Laboratory, Public Health England, Bristol, United Kingdom

Background and Aims: We recently reported a significant association between picornavirus (rhinovirus) infection and both rates and density of pneumococcal $(\mathrm{Sp})$ carriage in pre-school children.

Methods: In a further cross-sectional study in 586 children attending nurseries in Coimbra Portugal we took nasal swabs (into STGG) and recorded symptoms and demographics in March 2010. Samples underwent bacterial culture and RT-PCR for common respiratory viruses. Multivariate logistic regression analysis was done to find associations that were independent of age. Differences described were statistically significant $p<0.05$.

Results: Respiratory bacterial (Sp 59\%, Haemophilus influenzae (Hi) 52\%, Moraxella catarrhalis (Mc) 69\%) and viral (58\%) detection rates were high. There was a strong age-independent association between the presence and the density of $\mathrm{Sp}$ and the presence and severity of symptoms of rhinitis. Although weaker such associations were also observed for $\mathrm{Hi}$ and $\mathrm{Mc}$, the presence and density of these species was also significantly and age-independently associated with presence and density of $\mathrm{Sp}$. There was a highly significant negative age-independent association between Mc and Sa. In contrast to our previous study, clear associations between Sp and viral infection were not observed.

Conclusion: Nasal colonisation with $\mathrm{Sp}$ is commonly described as asymptomatic. This study suggests that this may be misleading but does not show whether Sp causes symptoms, rhinitis induces proliferation of Sp, or both (or neither). Nevertheless since successful colonisation and transmission are the two prerequisites for survival of Sp, it makes intuitive sense that its life cycle should be sensitive to the opportunity of a runny nose.

No conflict of interest 
ISPPD-0115

Pneumococcal Colonization and Carriage

\title{
PNEUMOCOCCAL DYNAMICS PRE-AND POST-VACCINATION: THE INTERPLAY BETWEEN SEROTYPE COMPETITION AND VACCINE EFFICACY
}

\author{
E. Gjini ${ }^{1}$, C. Valente ${ }^{2}$, R. Sá-Leão ${ }^{2}$, G. Gomes ${ }^{3}$ \\ ${ }^{1}$ Collective Dynamics, Institute Gulbenkian of Science, Oeiras, Portugal; ${ }^{2}$ Laboratory of Molecular Microbiology of Human Pathogens Institu- \\ to de Tecnologia Química e Biológica, Universidade Nova de Lisboa, Oeiras, Portugal; ${ }^{3}$ Collective Dynamics, Institute Gulbenkian Science, Oeiras, \\ Portugal
}

Background and Aims: The dynamics of Streptococcus pneumoniae exhibit variation worldwide both in serotype composition and absolute prevalence. Changes after vaccination targeting a subset of serotypes are bound to depend on the underlying transmission, competition and clearance rates, besides vaccine efficacy and coverage across host communities.

Methods: In this study, we model pneumococcus colonization dynamics in young children through a classical SIS epidemiological framework, accounting for single and multiple serotype carriage. Assuming functional symmetry between serotypes, we find a continuum of stable coexistence equilibria pre-vaccine, with varying serotype composition, but where the total prevalences of colonization and co-colonization are constrained by serotypetranscending parameters. The post-vaccination scenario is primarily determined by the proportion of the population vaccinated and the per-capita vaccine-induced protection; however these are dynamically integrated over time with a gradually diminishing exposure to vaccine serotypes (VT), and with the indirect effects of competition from non-vaccine serotypes (NVT).

Results: We fit these models to cross-sectional data from Portugal (2001-2007), linking mechanistically the pre- and post-vaccine era. Examining how the VT-NVT system re-adjusts post-vaccination, we find that serotype replacement occurs gradually, due to sustained positive feedback between vaccine protection against VT and general serotype competition. Vaccine efficacy, when accounting for such feedback, is around $16 \%(95 \% \mathrm{Cl}: 0.12-0.21)$, the relative rate of acquisition in already colonized hosts is $0.08(95 \% \mathrm{Cl}: 0.05-0.11)$, while the monthly per-capita transmission rate is 1.86 (95\% Cl: $1.65-2.07)$.

Conclusion: Our results emphasize the role of dynamical coupling between epidemiological variables when evaluating vaccine effects in multi-type bacterial communities.

No conflict of interest

ISPPD-0227

Pneumococcal Colonization and Carriage

\section{THE ROLE OF INFLUENZA AND PARAINFLUENZA INFECTIONS IN NASOPHARYNGEAL PNEUMOCOCCAL ACQUISITION AMONG YOUNG CHILDREN}

\author{
C.G. Grijalva ${ }^{1}$, M.R. Griffin ${ }^{1}$, K.M. Edwards ${ }^{2}$, J.V. Williams ${ }^{2}$, A.I. Gil ${ }^{3}$, H. Verastegui ${ }^{3}$, S.M. Hartinger $^{3}$, J.E. Vidal ${ }^{4}$, \\ K.P. Klugman ${ }^{5}$, C.F. Lanata ${ }^{3}$ \\ ${ }^{1}$ Health Policy, Vanderbilt University, Nashville, USA; ${ }^{2}$ Pediatrics, Vanderbilt University, Nashville, USA; ${ }^{3}$ IIN, Instituto de Investigacion Nutricional, \\ Lima, Peru; ${ }^{4}$ Rollins School of Public Health, Emory University, Atlanta, USA; ${ }^{5}$ Pneumonia, Bill \& Melinda Gates Foundation, Seattle, USA
}

Background and Aims: Since animal models suggest that influenza infection favors nasopharyngeal acquisition of pneumococci, we assessed this relationship with influenza and other respiratory viruses in young children. Methods: A case-control study was nested within a prospective cohort study of acute respiratory illness (ARI) in Andean children $<3$ years of age (RESPIRA-PERU study). Weekly household visits were made to identify ARI and obtain nasal swabs for viral detection using real-time PCR (RT-PCR). Monthly nasopharyngeal (NP) samples were obtained to assess pneumococcal colonization. We determined whether specific respiratory viral ARI occurring within the interval between consecutive NP samples (episodes of observation) increased the risk of nasopharyngeal acquisition of new pneumococcal serotypes.

Results: A total of 729 children contributed 2128 episodes of observation, including 681 pneumococcal acquisition episodes (new serotype compared to prior sample), 1029 non-acquisition episodes (no colonization or persistent colonization with the same serotype as the prior sample) and 418 indeterminate episodes. The risk of pneumococcal acquisition increased following influenza-ARI (adjusted odds ratio: 2.19, 95\% Cl: 1.02-4.69) and parainfluenza-ARI (1.86, 95\% Cl: 1.15-3.01), when compared with episodes without ARI. Other viral infections (respiratory syncytial virus, human metapneumovirus, human rhinovirus and adenovirus) were not associated with acquisition (Figure).

Conclusion: Influenza and parainfluenza ARIs appeared to facilitate pneumococcal acquisition among young children. As acquisition increases the risk of pneumococcal diseases, these observations are pivotal in our attempts to prevent pneumococcal disease. 


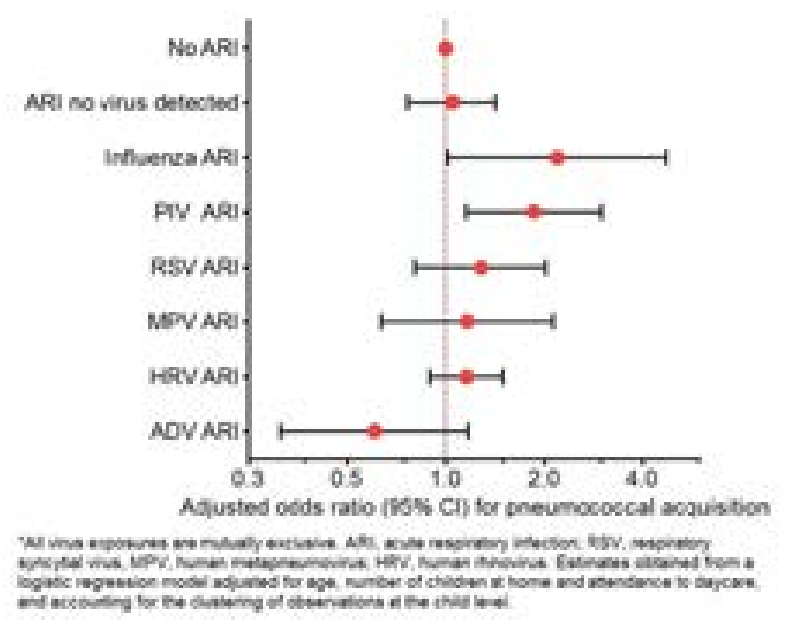

Conflict of interest

ISPPD-0076

Pneumococcal Colonization and Carriage

\title{
MULTI-SEROTYPE NASOPHARYNGEAL PNEUMOCOCCAL CARRIAGE PREVALENCE IN VACCINE NAÏVE NEPALESE CHILDREN, ASSESSED USING A MICROARRAY METHOD
}

R. Kandasamy ${ }^{1}$, M. Gurung ${ }^{2}$, S. Ndimah ${ }^{3}$, A. Thapa ${ }^{2}$, N. Adiakhari², M.D. Snape ${ }^{3}$, D.R. Murdoch ${ }^{4}$, D.F. Kelly ${ }^{3}$, S. Shrestha 2 , S. Thorson ${ }^{2}$, J. Hinds ${ }^{5}$, A.J. Pollard ${ }^{3}$

${ }^{1}$ Oxford Vaccine Group, University of Oxford, Oxford, United Kingdom; ${ }^{2}$ Paediatric Research Unit, Patan Academy of Health Sciences, Kathmandu, Nepal; ${ }^{3}$ Paediatrics, University of Oxford, Oxford, United Kingdom; ${ }^{4}$ Pathology, University of Otago, Christchurch, New Zealand; ${ }^{5}$ Cellular and Molecular Medicine, University of London, London, United Kingdom

Background and Aims: Nasopharyngeal carriage studies provide an insight into the local prevalence of circulating pneumococcal serotypes. There are very few data on the concurrent carriage of multiple pneumococcal serotypes. This study aimed to identify the prevalence and serotype distribution of pneumococci carried in the nasopharynx of young healthy Nepalese children prior to the introduction of a pneumococcal conjugate vaccine using a microarray method capable of detecting multi-serotype carriage.

Methods: We conducted a cross-sectional study of healthy children aged 6 weeks to 24 months from the Kathmandu Valley, Nepal between May and October 2012. Nasopharyngeal swabs were frozen and subsequently plated on selective culture media. Plate sweeps of pneumococcal colonies from these cultures were processed by DNA microarray capable of detecting relative abundance of multiple pneumococcal serotypes.

Results: 600 children were enrolled into the study: 199 aged 6 weeks to $<6$ months, 202 aged 6 months to $<12$ months, and 199 aged 12 months to 24 months. Pneumococci were identified in $51.5 \%$ of samples with more than one serotype being found in $30.4 \%$ of these samples. The serotypes covered by PCV13 were identified in $46 \%$ of samples containing pneumococci.

Conclusion: Application of a DNA microarray approach to identification of multiple pneumococcal serotype carriage demonstrates a substantial prevalence of co-colonisation. Continued surveillance utilising this approach following the introduction of routine use of pneumococcal conjugate vaccinates in infants will provide a more accurate understanding of vaccine efficacy against carriage and a better understanding of the dynamics of subsequent serotype and genotype replacement.

No conflict of interest

ISPPD-0036

Pneumococcal Colonization and Carriage

\section{A MODEL TO PREDICT THE PREVALENCE AND DISTRIBUTION OF COLONIZING PNEUMOCOCCI IN OLDER AGES AS A FUNCTION OF THAT IN YOUNG CHILDREN}

\author{
O. Le Polain de Waroux ${ }^{1}$, S. Flasche ${ }^{1}$, D. Prieto-Merino ${ }^{2}$, W.J. Edmunds ${ }^{1}$
}

${ }^{1}$ Department of Infectious Disease Epidemiology, London School of Hygiene \& Tropical Medicine, London, United Kingdom; ${ }^{2}$ Department of Medical Statistics, London School of Hygiene \& Tropical Medicine, London, United Kingdom

Background and Aims: Data on the nasopharyngeal carriage prevalence of Streptococcus pneumoniae across age groups are important to help predict the impact of introducing pneumococcal conjugate vaccines (PCVs) into routine vaccination programmes, given their important indirect effect. Yet most carriage studies are conducted in children $<5$ y only. We investigated whether carriage rates in young children could be used to infer those in older age groups. Methods: We conducted a systematic review of studies providing carriage estimates across age groups in healthy populations not previously exposed to PCV, using MEDLINE and Embase. We used Bayesian linear meta-regression 
models to predict the carriage prevalence - overall as well as vaccine serotypes (VT) and non-vaccine serotypes (NVT) - in older age groups as a function of that in $<5 \mathrm{y}$ olds.

Results: Twenty-nine studies were included in the analysis, with a total of 20,391 individuals. In all studies nasopharyngeal carriage decreased with increasing age. We found a strong positive association between the prevalence in children $<5 \mathrm{y}$ and that in school aged children $(5-17 \mathrm{y}$ olds) and in adults. The proportion of VT serotypes among carriers was on average about $73 \%$ that of children $<5 y$ in both $5-17$ y olds and adults. We provide a simple prediction model to infer the carriage prevalence in 5-17 y olds and adults as a function of that in young children.

Conclusion: Where only carriage information in young children is available, such model can be used to infer carriage rates in other age groups and help predict the potential population-wide effects of PCV.

No conflict of interest

ISPPD-0041

Pneumococcal Colonization and Carriage

\title{
ANTIBODY BLOCKS ACQUISITION OF PNEUMOCOCCAL COLONIZATION THROUGH AGGLUTINATION
}

\author{
A.M. Roche ${ }^{1}$, A.L. Richard ${ }^{1}$, J.T. Rahkola², E.N. Janoff ${ }^{2}$, J.N. Weiser ${ }^{1}$ \\ ${ }^{1}$ Department of Microbiology, University of Pennsylvania Perelman School of Medicine, Philadelphia, USA; ${ }^{2}$ Division of Infectious Disease, MA- \\ VRC University of Colorado Denver, Aurora, USA
}

Background and Aims: A major benefit of the pneumococcal conjugate vaccine has been attributed to herd protection, resulting from its ability to decrease transmission by blocking the acquisition of colonization. Mucosal protection against colonization correlates with increased serum anti-capsular antibody $(A b)$ titers, however, the mechanism for this protection is unknown.

Methods: A murine model was used to study the dynamics of protection against colonization. Mice were passively immunized with rabbit anti-pneumococcal Ab or type-specific human monoclonal IgA and challenged intranasally with S. pneumoniae 4 hours later. Colonization was assessed 20 hours post challenge.

Results: Passively immunized mice were protected from acquisition of colonization. IgG detected on the mucosa at the time of nasal challenge was sufficient to block acquisition. The effectiveness of IgG was independent of Fc-fragment and complement, suggesting an effect of Ab other than opsonophagocytosis. Protection was serotypespecific and correlated with the agglutinating effect of anti-sera. Immunization with $F\left(a b^{\prime}\right)$, fragments (agglutinating) was more protective than Fab fragments (non-agglutinating). In humans, IgA1 is the major immunoglobulin on the nasal mucosa, but it is cleaved in the hinge region by a pneumococcal protease, eliminating its ability to agglutinate. Serotype-specific hlgA1 mAb blocked colonization of an IgA1-protease mutant (agglutinated), but not the proteaseproducing wild-type parent (not agglutinated). Protease-resistant hlgA2 mAb protected against both strains. Finally, agglutinating Abs blocked transmission between littermates in an infant model using influenza co-infection. Conclusion: The agglutinating effect of Ab promotes mucosal protection against S. pneumoniae colonization and demonstrates a mechanism for PCV-induced mucosal protection.

No conflict of interest

\section{ISPPD-0385 \\ Pneumococcal Colonization and Carriage}

\section{CO-OCCURRENCE PATTERNS OF STREPTOCOCCUS PNEUMONIAE SEROTYPES WITH OTHER MICROBIAL INHABITANTS OF THE NASOPHARYNX IN CHILDREN}

\author{
G. Biesbroek ${ }^{1}$, K. Trzcinski ${ }^{1}$, X. Wang ${ }^{1}$, B.J.F. Keijser ${ }^{2}$, R.H. Veenhoven ${ }^{3}$, E.A.M. Sanders ${ }^{1}$, D. Bogaert ${ }^{1}$
}

${ }^{1}$ Pediatric Immunology and Infectious Diseases, UMC Utrecht, Utrecht, Netherlands; ${ }^{2}$ Research Group Microbiology and Systems Biology, TNO Earth Environment and Life Sciences, Zeist, Netherlands; ${ }^{3}$ Research Centre Linnaeus Institute, Spaarne Hospital, Hoofddorp, Netherlands

Background and Aims: Streptococcus pneumoniae serotypes vary in their phenotypically characteristics including carriage prevalence and invasiveness in the population. Interactions between different pneumococcal serotypes and other colonizing bacteria might contribute to this process. We therefore studied the co-occurrence of the most common pneumococcal serotypes with other bacteria in the nasopharyngeal microbiota.

Methods: The nasopharyngeal microbiota of 800 healthy children at the ages of either 1.5, 6, 12 and 24 months was characterized by 16 S GS-flx-titanium-pyrosequencing. Pneumococci were cultured and serotyped using the Quellung reaction.

Results: The relative abundance of $S$. pneumoniae differed significantly between serotypes, ranging from 6-7\% for serotypes $6 \mathrm{~A}$ and $35 \mathrm{~A} / \mathrm{B}$ to approx. $20 \%$ for serotypes $6 \mathrm{~B}$ and $23 \mathrm{~A} / \mathrm{B}$. An inverse relationship was observed for cooccurrence patterns of these serotypes with Dolosigranulum and Corynebacterium yet the most clear dichotomy in co-occurrence was observed with Staphylococccus ranging from $<0.1 \%$ in the presence serotypes $23 \mathrm{AB}$ to $7 \%$ in the presence of $35 \mathrm{~A} / \mathrm{B}$. Heamophilus abundance also varied with pneumococcal serotypes present ranging from $5 \%$ (serotype $19 \mathrm{~A}$ ) to $13 \%$ (serotype $16 \mathrm{~A}$ ). In addition, we observed significant different microbiota profiles in the presence of serotype 11A and non-typeable (NT) pneumococci compared to all other serotypes with higher abundances of Moraxella, non-pneumococcal Streptococci, Gemella, Prevotella and Fusobacterium in the presence of serotype $11 \mathrm{~A}$ and high abundances of Staphylococcus, non-pneumococcal and Corynebacterium in the presence of NT $(p<0.001)$ 
Discussion: The current study suggests differences in microbial co-occurrence patterns between pneumococcal serotypes potentially contributing to differences in phenotypical characteristics of individual serotypes. No conflict of interest

ISPPD-0277

Pneumococcal Colonization and Carriage

\section{RETURN TO PRE-PCV CARRIAGE LEVELS OF STAPHYLOCOCCUS AUREUS 6.5 YEARS AFTER PNEUMOCOCCAL CONJUGATE VACCINE IN THE DUTCH NATIONAL IMMUNIZATION PROGRAM}

A.A.T.M. Bosch ${ }^{1}$, R.H. Veenhoven ${ }^{2}$, J.P. Bruin ${ }^{3}$, A.J. Wijmenga-Monsuur ${ }^{4}$, K. Trzcinski ${ }^{1}$, A. Wyllie ${ }^{1}$, D. Bogaert ${ }^{1}$, N.Y. Rots ${ }^{4}$, E.A.M. Sanders ${ }^{1}$

${ }^{1}$ Pediatric Immunology and Infectious Diseases, Wilhelmina Children's Hospital University Medical Center Utrecht, Utrecht, Netherlands;

${ }^{2}$ Research Center Linnaeus Institute, Spaarne Hospital and Kennemer Gasthuis, Hoofddorp and Haarlem, Netherlands; ${ }^{3}$ Regional Laboratory of, Public Health, Haarlem, Netherlands; ${ }^{4}$ Center for Immunology of Infectious Diseases and Vaccines, National Institute of Public Health and the Environment, Bilthoven, Netherlands

Background: Increased carriage of Staphylococcus aureus in 11-month-old children and of Haemophilus influenzae and Moraxella catarrhalis in young children and their parents after implementation of pneumococcal conjugate vaccines (PCV) raised concerns about the impact of PCV on non-pneumococcal respiratory infectious diseases(vanGils,2011; Spijkerman,2012). We performed a cross-sectional study to investigate long-term effects of vaccination on nasopharyngeal carriage of bacterial pathogens 6.5 years after PCV7-implementation and 1.5 years after PCV7 was replaced by PCV10.

Methods: Nasopharyngeal carriage rates of S. aureus, H. influenzae, and $M$. catarrhalis were determined in 330 PCV7-vaccinated 24-month-old children and their parents and 330 PCV10-vaccinated 11-month-old children using conventional culture. Current results were compared with historical data from studies conducted before, and 3 and 4.5 years after PCV7-implementation.

Results: After an increase following PCV-introduction, carriage of S. aureus in 11-month-old children and parents of 24-month-old children had decreased compared to 4.5 years post-PCV7 (adjusted odds ratio (aOR) $0.47(0.28$ 0.80 ) and aOR 0.59(0.42-0.84)), and was comparable to pre-PCV7 carriage rates. Carriage of $H$. influenzae and $M$. catarrhalis remained stable after PCV-implementation, i.e. $61 \%, 68 \%$ and $17 \%$ for $H$. influenzae, and $76 \%, 73 \%$ and $18 \%$ for M. catarrhalis in 11-month-old and 24-month-old children and parents, respectively.

Conclusion: 6.5 years after PCV7-implementation and 1.5 years after replacement by PCV10, nasopharyngeal carriage of $S$. aureus declined towards pre-vaccination levels. Introduction of PCV10 did not seem to have affected overall $H$. influenzae colonization. Our data may reflect a new balance in the interplay between non-vaccine pneumococci, which had completely replaced vaccine serotypes, and other co-habitants of the nasopharyngeal niche.

No conflict of interest

ISPPD-0478

Pneumococcal Colonization and Carriage

\section{REDUCTIONS IN PCV13-TYPE COLONIZATION AMONG IMMUNIZED AND UNIMMUNIZED AMERICAN INDIAN COMMUNITY MEMBERS}

L. Grant ${ }^{1}$, S. O'Brien ${ }^{1}$, R. Weatherholtz ${ }^{1}$, M. Jacobs ${ }^{2}$, S. Bajaksouzian ${ }^{2}$, R. Reid ${ }^{1}$, M. Santosham ${ }^{1}$, K. O'Brien ${ }^{1}$

${ }^{1}$ Center for American Indian Health, Johns Hopkins Bloomberg School of Public Health, Baltimore, USA; ${ }^{2}$ University Hospitals Case Medical Center, Case Western Reserve University, Cleveland, USA

Background and aims: The 13-valent pneumococcal conjugate vaccine (PCV13) replaced PCV7 in March 2010 on American Indian (AI) reservations in the Southwest United States. We evaluated the impact of PCV13 on vaccinetype (VT) nasopharyngeal (NP) colonization among vaccinated and unvaccinated community members.

Methods: From January 2010-April 2012, an NP specimen was collected from each participating Al household member of a prospective, continuous, cross-sectional colonization study. Pneumococci were isolated by culture following broth enrichment and serotyped by Quellung. Chi-square test for linear trend measured VT colonization prevalence across the study period. The 13 serotypes in PCV13 were defined as VTs.

Results: A total of 6645 individuals from 1821 households enrolled. An NP specimen was collected from 6628 individuals (99.7\%); 2187 (33\%) were pneumococcus positive (<5 y: 52\%; 5-17 y: $36 \% ; 18+y: 12 \%)$. VT colonization prevalence decreased from $12 \%$ prior to PCV13 introduction to $4 \%$ for immunized ( $p=0.01$ ) and $5 \%$ for unimmunized children $<5$ y $(p=0.4)$ two years later (March 2012). Serotype 6 C decreased significantly for $<5$ y $(5 \%-2 \%, p=0.01)$, but not for older ages. VT prevalence decreased (but not statistically significant) for those 5-17 y $(10 \%-3 \%, p=0.11)$ and adults $18+\mathrm{y}(2 \%-1 \%, p=0.07)$. Serotypes $19 \mathrm{~A}$ and $6 \mathrm{C}$ ranked among the top 10 most prevalent serotypes in year two of the study for all ages (<5 y: $2 \%$ each; $5-17$ y: $2 \%$ each; $18+y: 0.3 \%$ each). Conclusion: VT colonization decreased at an average of $5 \%$ with each successive month of the study, including those unimmunized with PCV13. VT colonization continued to be detected in the second year of PCV13 use.

Conflict of interest 


\section{POPULATION IMPACT OF 10-VALENT PNEUMOCOCCAL CONJUGATE VACCINE ON NASOPHARYNGEAL CARRIAGE} OF STREPTOCOCCUS PNEUMONIAE AND NON-TYPEABLE HAEMOPHILUS INFLUENZAE IN KILIFI, KENYA

L.L. Hammitt ${ }^{1}$, D.O. Akech², S.C. Morpeth ${ }^{2}$, A. Karani ${ }^{2}$, N. Kihuha ${ }^{2}$, S. Nyongesa ${ }^{2}$, T. Bwanaali² ${ }^{2}$ E. Mumbo ${ }^{3}$, T. Kamau $^{4}$, S.K. Sharif ${ }^{5}$, J.A.G. Scott ${ }^{6}$

${ }^{1}$ International Health, Johns Hopkins Bloomberg School of Public Health, Baltimore, USA; ${ }^{2}$ Epidemiology, KEMRI-Wellcome Trust Research Programme, Kilifi, Kenya; ${ }^{3}$ Kilifi District, Kenya Ministry of Health, Kilifi, Kenya; ${ }^{4}$ Vaccines and Immunization, Kenya Ministry of Health, Nairobi, Kenya ${ }^{5}$ Public Health, Kenya Ministry of Health, Nairobi, Kenya; ${ }^{6}$ Infectious Disease Epidemiology, London School of Hygiene and Tropical Medicine, London, United Kingdom

Background: The impact of 7-valent pneumococcal conjugate vaccine (PCV) in developed countries was enhanced by indirect protection of unvaccinated individuals, mediated by reduced nasopharyngeal carriage of vaccineserotype pneumococci. The potential indirect protection of 10-valent PCV (PCV10) in a developing country setting is unknown. We sought to estimate the effectiveness of programmatic introduction of PCV10 in Kenya against carriage of vaccine-serotype pneumococci and non-typeable Haemophilus influenzae (NTHi).

Methods: PCV10 was introduced into the infant vaccination program in Kenya in January 2011, accompanied by a catch-up campaign in Kilifi County for children $<5$ years. We conducted annual cross-sectional studies of carriage among an age-stratified, random population sample in the 2 years before and 2 years after PCV10 introduction. Approximately 500 individuals were enrolled each year. Carriage prevalence ratios in the 2 years following PCV10 introduction compared to baseline were determined by log-binomial regression.

Results: Among children aged $<5$ years the prevalence of vaccine-serotype, non-vaccine-serotype and NTHi carriage was $0.34,0.41$ and 0.54 , respectively, at baseline and $0.13,0.57$ and 0.40 , respectively, after PCV10 introduction. Adjusted prevalence ratios were 0.36 (95\%Cls: $0.26-0.51), 1.37$ (1.13-1.64) and 0.62 (0.52-0.75), respectively. Among individuals $\geq 5$ years, the adjusted prevalence ratios for vaccine-serotype pneumococci and NTHi were 0.34 (95\%Cls: 0.18-0.62) and 0.71 (0.56-0.89), respectively.

Conclusion: Following programmatic use of PCV10 in Kilifi, carriage of vaccine-serotypes was reduced by $64 \%$ in children $<5$ years and $66 \%$ in older individuals. These findings suggest that PCV10 introduction in Africa will have a substantial indirect effect on invasive pneumococcal disease.

Conflict of interest

ISPPD-0324

Pneumococcal Colonization and Carriage

\section{EFFECTS OF 10-VALENT PNEUMOCOCCAL NON-TYPEABLE HAEMOPHILUS INFLUENZAE PROTEIN D CONJUGATE VACCINE (PHID-CV) ON THE NASOPHARYNGEAL MICROBIOME OF KENYAN TODDLERS}

D. Frank ${ }^{1}$, L.M. Feazel ${ }^{2}$, S.A. Santorico ${ }^{3}$, C.E. Robertson ${ }^{4}$, A. Karani ${ }^{5}$, M. Bashraheil ${ }^{5}$, J.A.G. Scott ${ }^{5}$, L.L. Hammitt ${ }^{6}$

${ }^{1}$ Division of Infectious Diseases, University of Colorado School of Medicine, Aurora, USA; ${ }^{2}$ Carver College of Medicine, University of lowa, lowa City, USA; ${ }^{3}$ Department of Mathematical \& Statistical Sciendes, University of Colorado Denver, Denver, USA; ${ }^{4}$ Department of Molecular Cellular and Developmental Biology, University of Colorado Boulder, Boulder, USA; ${ }^{5}$ Centre for Geographic Medicine-Coast, KEMRIWellcome Trust Research Programme, Kilifi, Kenya; ${ }^{6}$ Department of International Health, Johns Hopkins Bloomberg School of Public Health, Baltimore, USA

Background: Pneumococcal conjugate vaccines reduce the incidence of invasive pneumococcal disease and decrease nasopharyngeal colonization by vaccine serotypes. Because changes in colonization by potential pathogens could alter patterns of invasive disease, we monitored the nasopharyngeal microbiome of children following vaccination with 10-valent pneumococcal non-typeable Haemophilus influenzae protein-D conjugate vaccine (PHiD-CV).

Methods: Children aged 12-59 months were randomized to receive 2 doses of PHiD-CV $(n=30)$ or Hepatitis A ( $n=$ 30) vaccine 2 months apart. Nasopharyngeal swabs were collected prior to vaccination and 180 days after the first dose. The nasopharyngeal microbiome was profiled by 16S rRNA gene pyrosequencing of swab specimens. Results: Streptococcus pneumoniae (19.5\% of sequences), Moraxella catarrhalis (14.4\%), and Corynebacterium spp. (8.5\%) were the most abundant nasopharyngeal bacteria. Vaccination with PHiD-CV did not result in significant differences in species composition, abundance, or prevalence of known pathogens. Distinct microbiome compositions were identified based on relative abundance of Streptococcus, Moraxella, and Haemophilus species. These microbiomes were not stable over the 180-day study period and were independent of age, sex, water source, school attendance, and vaccination.

Conclusions: Vaccination of toddlers with two doses of PHiD-CV did not significantly alter the composition of the nasopharyngeal microbiome. This suggests limited opportunity for replacement colonization with other pathogens but does not preclude replacement with non-vaccine strains of $S$. pneumoniae.

Conflict of interest 
INDIRECT EFFECTIVENESS OF PNEUMOCOCCAL HAEMOPHILUS INFLUENZAE PROTEIN-D CONJUGATE VACCINE (PHID-CV10) AGAINST OROPHARYNGEAL AND NASOPHARYNGEAL CARRIAGE - FINIP INDIRECT CARRIAGE STUDY

J. Jokinen ${ }^{1}$, T.M. Kilpi ${ }^{1}$, T. Kaijalainen ${ }^{2}$, R. Syrjänen ${ }^{3}$, E. Ruokokoski ${ }^{1}$, M. Van Dyke ${ }^{4}$, A.A. Palmu ${ }^{3}$

${ }^{1}$ Department of Vaccination and Immune Protection, National Institute for Health and Welfare, Helsinki, Finland; ${ }^{2}$ Department of Vaccination and Immune Protection, National Institute for Health and Welfare, Oulu, Finland; ${ }^{3}$ Department of Vaccination and Immune Protection, National Institute for Health and Welfare, Tampere, Finland; ${ }^{4}$ GlaxoSmithKline Vaccines, Wavre, Belgiu

Background: FinIP trial was designed to evaluate effectiveness of PHiD-CV10 (GlaxoSmithKline) against diseases associated with Streptococcus pneumoniae and Haemophilus influenzae. We conducted a satellite study in older siblings of the FinIP-vaccinated children to evaluate indirect effectiveness against carriage. Previously presented results based on nasopharyngeal samples demonstrated $29 \%$ reduction in vaccine-type carriage. Here we report results based on both nasopharyngeal (NPS) and orophragyngeal (OPS) samples.

Methods: FinIP was a cluster-randomised, double-blind trial, where 29126 children $<7$ months were recruited from February 2009 - October 2010. Children received PHiD-CV10 in 2/3 and control vaccine in $1 / 3$ of 72 clusters according to $3+1$ or $2+1$ schedules. For our indirect carriage study, we sampled separately the NPS and the OPS of 1423 unvaccinated 3 to 7-year-old siblings of FinIP participants from Apr'11 to Nov'11. Generalized linear mixed model was used to estimate indirect effectiveness against carriage due to S. pneumoniae (Pnc), $H$. influenzae, Moraxella catarrhalis and Staphylococcus aureus.

Results: Positive in either NPS or OPS increased the pneumococcal yield by $25 \%$ compared to NPS-alone. Table reports indirect vaccine effectiveness (VE) of PHiD-CV10 against NPS-alone / NPS or OPS carriage (1-odds ratios) in unvaccinated siblings of the FinIP-vaccinated children.

\begin{tabular}{|c|c|c|c|c|c|c|c|c|}
\hline \multirow[b]{2}{*}{ Pathogen } & \multicolumn{3}{|c|}{$\begin{array}{l}\text { N positives for } \\
\text { NPS / NPS er OPS }\end{array}$} & \multicolumn{3}{|c|}{$\begin{array}{l}\text { Camiage percentage for } \\
\text { NPS / NPS ar ORs }\end{array}$} & \multicolumn{2}{|c|}{$\begin{array}{l}\text { VE for PHiD-CVI0 } \\
\text { gruups cumbined }\end{array}$} \\
\hline & $\begin{array}{l}\text { Control } \\
(\mathrm{N}-\mathrm{SON})\end{array}$ & $\begin{array}{l}\text { PHid } \\
\text { CVI0 } \\
2+1 \\
(N-471)\end{array}$ & $\begin{array}{l}\text { PliD } \\
\text { CV10 } \\
3+1 \\
(N=43 y)\end{array}$ & Control & $\begin{array}{l}\text { PHiD- } \\
\text { CVI0 } \\
2+1\end{array}$ & $\begin{array}{l}\text { PHD. } \\
\text { CV10 } \\
3 * 1\end{array}$ & $\begin{array}{l}\text { Against } \\
\text { NPS } \\
\text { Estimnte } \\
\text { (95\%C1) }\end{array}$ & $\begin{array}{l}\text { Against } \\
\text { NPS or OPS } \\
\text { Estimate } \\
\text { (95\%Cl) }\end{array}$ \\
\hline Overall Phe & $197 / 237$ & $155 / 213$ & $174 / 205$ & $38 / 47$ & 3245 & $39 / 47$ & $10(-12,28)$ & $2(-22,21)$ \\
\hline $\begin{array}{l}\text { Vactias-type } \\
\text { Pne }\end{array}$ & $100 / 125$ & $60 / 90$ & $65 / 79$ & $19 / 25$ & $14 / 19$ & $14 / 18$ & $29(6,47)$ & $29(8,46)$ \\
\hline H.infucturac & $71 / 96$ & $63 / 79$ & $54 / 74$ & $14 / 19$ & $13 / 17$ & $12 / 17$ & $9(-25,34)$ & $11(-21,35)$ \\
\hline Saureus & $112 / 245$ & $124 / 225$ & $104 / 207$ & $22 / 48$ & $26 / 48$ & $23 / 48$ & $-17(-52.9)$ & $2(-23,21)$ \\
\hline M.eatarthalis & $175 / 185$ & $158 / 165$ & $160 / 168$ & $34 / 36$ & $33 / 35$ & $36 / 39$ & $-5(-40,20)$ & $-5(-41,22)$ \\
\hline
\end{tabular}

Conclusion: Impact against vaccine-type carriage was identical for NPS-alone and with the addition of OPS. Addition of OPS increases sensitivity of detection of pneumococcus and their combination may therefore give a more accurate estimate of carriage prevalence.

Conflict of interest

ISPPD-0328

Pneumococcal Colonization and Carriage

\title{
HOW DOES THE PROBIOTIC STREPTOCOCCUS SALIVARIUS INHIBIT PNEUMOCOCCAL ADHERENCE IN VITRO?
}

\author{
J. Manning ${ }^{1}$, E.M. Dunne ${ }^{1}$, E.K. Mulholland ${ }^{1}$, R.M. Robins-Browne ${ }^{2}$, C. Satzke ${ }^{1}$ \\ ${ }^{1}$ Pneumococcal Research, Murdoch Childrens Research Institute, Parkville Melbourne, Australia; ${ }^{2}$ Department of Microbiology and Immunology, \\ The University of Melbourne, Parkville Melbourne, Australia
}

Background and Aims: Pneumococcal nasopharyngeal colonisation precedes infection and disease. Mounting evidence suggests probiotic bacteria could play a role in reducing disease in the respiratory tract. We previously demonstrated that the probiotic Streptococcus salivarius inhibits pneumococcal adherence to epithelial cells in vitro. This study investigates the mechanism of inhibition, including the role of megaplasmid-encoded bacteriocins (antibacterial peptides).

Methods: Several S. salivarius strains harbouring megaplasmids (encoding several bacteriocins), and their megaplasmid-negative derivatives, were tested for the ability to inhibit pneumococcal growth on solid media, and their adherence to human pharyngeal epithelial cells (Detroit 562). Pneumococcal adherence was compared following 1) pre-administration of $S$. salivarius, 2) pre-administration followed by removal of non-adherent $S$. salivarius, and 3) pre-administration of S. salivarius into permeable transwell inserts.

Results: Only megaplasmid-positive $S$. salivarius inhibited pneumococcal growth on solid media, however, both megaplasmid-positive and negative strains of $S$. salivarius inhibited pneumococcal adherence. Inhibition was unaffected by removal of unbound $S$. salivarius following pre-administration. No inhibition was observed in 
transwell experiments in preliminary experiments.

Conclusion: Results suggest $S$. salivarius-mediated inhibition of pneumococcal adherence is not megaplasmiddependant, and requires $S$. salivarius contact with host cells. As such, inhibition of pneumococcal adherence is likely to be mediated by competition for binding, rather than by megaplasmid-encoded bacteriocins. This work may facilitate the development of novel strategies for the prevention of pneumococcal colonisation and disease.

Conflict of interest

ISPPD-0269

Pneumococcal Colonization and Carriage

\section{THE IMPACT OF PNEUMOCOCCAL CONJUGATE VACCINE (PCV) ON THE EPIDEMIOLOGY OF INVASIVE PNEUMOCOCCAL DISEASE (IPD) AND PNEUMOCOCCAL CARRIAGE IN SOWETO}

S.A. Nzenze ${ }^{1}$, T. Shiri ${ }^{1}$, L. de Gouveia ${ }^{2}$, A. Violari ${ }^{3}$, M.C. Nunes ${ }^{1}$, A. Karstaedt ${ }^{4}$, S.A. Madhi' ${ }^{2}$, A. von Gottberg ${ }^{2}$

${ }^{1}$ Department of Science and Technology/Vaccine Preventable Diseases Unit, Respiratory and Meningeal Pathogens Unit, Johannesburg, South Africa; ${ }^{2}$ National Institute of Communicable Diseases, Respiratory and Meningeal Pathogens Unit, Johannesburg, South Africa; ${ }^{3}$ University of the Witwatersrand, Perinatal HIV Research Unit, Johannesburg, South Africa; ${ }^{4}$ Department of Medicine, University of the Witwatersrand and Chris Hani Academic Hospital, Johannesburg, South Africa;

Background: The PCV reduces transmission of vaccine-serotypes. We assess the impact of PCV vaccination on carriage and IPD in Soweto.

Methods: Seven-valent PCV was introduced into the public immunization program for infants in April 2009 and replaced by 13-valent PCV from May 2011. Two pneumococcal carriage surveys were done in Soweto from January 2007 to May 2009 (Period-1) and May 2012 to April 2013 (Period-2) among mother-infant pairs. IPD cases for the same population were analyzed from the national surveillance database for pre-vaccine years (2005 to 2008) and for 2012 (post-vaccine). We compared disease incidences as well as carriage prevalence of PCV7+6A (vaccine serotype, VT), additional PCV13 serotypes (1, 3, 5, 7F, 19A) and non-vaccine serotypes (NVT) between the two periods. Additionally, we compared observed to expected IPD incidence calculated using a theoretical model. Results: VT decreased in Period-2 for both carriage and IPD among children (<2yrs) and females aged 15-45 years. Among children additional PCV13 serotypes decreased in IPD but not in carriage while NVTs increased in carriage but not in IPD, Table 1. Expected IPD was similar to observed IPD for adults.

Conclusion: VT decreased for carriage and IPD although \% reduction was greater in IPD, especially for children. VT colonization endpoints may approximate IPD endpoints among unvaccinated individuals and can potentially be used in post-vaccine introduction studies. Changes in NVT IPD may lag behind colonization.

\begin{tabular}{|c|c|c|c|c|c|c|c|c|}
\hline \multirow[t]{2}{*}{ Age group } & \multirow{2}{*}{$\begin{array}{l}\text { Preumcosccal } \\
\text { Serotypes }\end{array}$} & \multicolumn{3}{|c|}{ Camiage } & \multicolumn{3}{|c|}{ Inasive Preurococcal Disease } & \multirow{2}{*}{$\begin{array}{l}\text { Expectad } \\
\text { IPD } \\
\text { Incidence } \\
\text { (per } \\
\text { 100000)" }\end{array}$} \\
\hline & & $\begin{array}{l}\mathrm{n}(\mathrm{(6)}) \text { colenized } \\
\text { Pre-Neccine } \\
(2007 \cdot 2009) \\
\text { Period-1 }\end{array}$ & $\begin{array}{l}n(\%) \text { coloniced } \\
\text { Post-raccire } \\
\text { (2012) } \\
\text { Period-2 }\end{array}$ & $\begin{array}{l}\text { Relative Pisk } \\
(95 \% \mathrm{Cl})\end{array}$ & $\begin{array}{l}\text { Average } \\
\text { Incidence (ger } \\
100000 \text { ) } 200515 \\
2000: \text { Period-1 }\end{array}$ & $\begin{array}{l}\text { Inoidence } \\
\text { (per 100000) } \\
2012 \text { : Period. } \\
2\end{array}$ & $\begin{array}{l}\text { incidence risk } \\
\text { reduction } \\
\text { (95\% } \\
\text { Confidenot } \\
\text { interval) }\end{array}$ & \\
\hline \multirow[t]{4}{*}{$\begin{array}{l}\text { Childen } \\
\text { (2-2 years) }\end{array}$} & 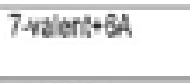 & $661(35.9)$ & $88(11.2)$ & $\begin{array}{c}0.31 \\
(0.25 \cdot 0.38)\end{array}$ & 149.0 & 14.5 & $\begin{array}{c}0.10 \\
0.06-2.17)\end{array}$ & 48.5 \\
\hline & $1,3,5,7 \mathrm{~F}, 12 \mathrm{~A}$ & $65(35)$ & $23(29)$ & $\begin{array}{c}0.83 \\
(0.52-1.32)\end{array}$ & 321 & 83 & $\begin{array}{c}0.26 \\
(0.120 .55) \\
\end{array}$ & 286 \\
\hline & NVT & $370(20.1)$ & $397(428)$ & $\begin{array}{c}2.13 \\
{[1.39-241)}\end{array}$ & 37.7 & 37.3 & $\begin{array}{c}0.99 \\
0.63-1.56)\end{array}$ & 80.3 \\
\hline & All preumococei & $1098(595)$ & $443(589)$ & $\begin{array}{c}0.96 \\
(0.80-1.08)\end{array}$ & 2009 & 602 & $\begin{array}{c}0.27 \\
0.20-0.35)\end{array}$ & 2112 \\
\hline \multirow{4}{*}{$\begin{array}{l}\text { Women } \\
\text { (15 to } 45 \\
\text { years) }\end{array}$} & 7 -velents+24 & $129(7.0)$ & $22(1.4)$ & $\begin{array}{c}0.20 \\
(0.13-0.32)\end{array}$ & 135 & 38 & $\begin{array}{c}0.28 \\
0.090 .87)\end{array}$ & 27 \\
\hline & $1,3,5,7 F, 19 A$ & $41(2.2)$ & $27(1.8)$ & $\begin{array}{c}0.79 \\
0.49 .128)\end{array}$ & 11.7 & 63 & $\begin{array}{c}0.54 \\
0.20-142)\end{array}$ & 9.6 \\
\hline & $N \sqrt{T}$ & $175(95)$ & $127(\mathrm{~B} 3)$ & $\begin{array}{c}0.87 \\
0.70-100)\end{array}$ & 7.1 & 97 & $\begin{array}{c}1.36 \\
0.52 .358)\end{array}$ & 62 \\
\hline & Al preumococei & $345(18.7)$ & $176(11.5)$ & $\begin{array}{c}0.61 \\
(0.520 .73)\end{array}$ & 323 & 198 & $\begin{array}{c}0.61 \\
0.35 \cdot 1.07)\end{array}$ & 19.9 \\
\hline
\end{tabular}

\section{The expected PD is equal to the proporion of pest vaccine carriage prevalense and pre-vacoine carriage prevalence mubipled by the pre-vacoine PD incidence}

No conflict of interest 


\title{
DIFFERENTIAL PCV7 EFFECT ON PILIATED AND NON-PILIATED VACCINE-TYPE STREPTOCOCCUS PNEUMONIAE (SP) CARRIED STRAINS
}

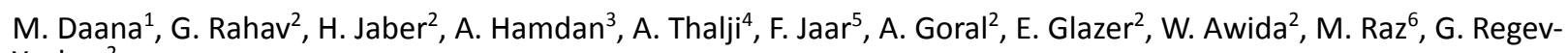
Yochay $^{2}$

${ }^{1}$ Primary Care, Maccabi Healthcare Services, Jerusalem, Israel; ${ }^{2}$ Infectious Disease Unit, Sheba Medical Center, Ramat Gan, Israel; ${ }^{3}$ Primary Care, Private Clinic, Nabulus, Palestine (via Israel); ${ }^{4}$ Primary Care, Private Clinic, Ramalla, Palestine (via Israel); ${ }^{5}$ Primary Care, Private Clinic, Bethlehem, Palestine (via Israel); ${ }^{6}$ Jerusalem-Hashfela Dis., Maccabi Healthcare Services, Modiin, Israel

Background: Type1 pilus proteins are potential targets of future protein-based vaccines. Yet, piliated strains were reported to decrease soon after PCV implementation. Here we report a differential effect of PCV7 on carriage of non-piliated strains in a population-based study comparing a vaccinated vs. nonvaccinated population before and after PCV7 implementation.

Methods: Consecutive annual cross-sectional surveys of SP carriage among children $<3.5 y$ were performed from 2009 (pre-PCV) to 2011 (post-PCV). Palestinian children from a) Ramalla, Nabulus and Bethlehem living under Palestinian-Authority's (PA) health policy, where PCV7 was not yet implemented and b) East-Jerusalem (EJ) where PCV7 was implemented (7/2009). Clinical data were collected and SP/serotype/antibiotic susceptibilities were identified. Presence of Type1 pilus was determined by $\operatorname{rrg} C$ PCR.

Results: A total of 983 and 1772 children from EJ and PA were screened. SP carriage was 30\% and was not affected by vaccination policy (after adjusting for age/region/year/recent antibiotic treatment). Carriage of VT7 strains decreased significantly following PCV implementation ( $52 \%$ to $22 \%, p<0.001$ ). The decrease was mainly of nonpiliated VT strains, while piliated-VT strains did not decrease. Piliated strains consisted $50 \%$ of all VT in 2009 vs. $80 \%$ in 2011, $(p<0.001)$. In addition, piliated non-VT13 strains emerged, from $1 \%$ (2009) to 25\% (2011). The emerging nonVT13 piliated serotypes were 35B, 11A and 19B. PCV implementation was associated with increased carriage of piliated strains (adjusted odds ratio (aOR): 2.6, 95\% Cl: 1.2-6.4).

Conclusion: The differential effect of PCV7 on non-piliated strains suggests that Type1 pilus confers an intrinsic advantage for colonization and that pilus proteins are attractive vaccine targets.

No conflict of interest

ISPPD-0136

Pneumococcal Colonization and Carriage

\section{PNEUMOCOCCAL CARRIAGE IN CHILDREN AND ADULTS TWO YEARS AFTER INTRODUCTION OF THE THIRTEEN VALENT PNEUMOCOCCAL CONJUGATE VACCINE IN ENGLAND}

\author{
A.J. van Hoek ${ }^{1}$, C. Sheppard ${ }^{2}$, N. Andrews ${ }^{1}$, P. Kaye ${ }^{1}$, T. Harrison ${ }^{2}$, M. Slack ${ }^{2}$, S. Ladhani ${ }^{1}$, E. Miller ${ }^{1}$ \\ ${ }^{1}$ Immunisation Hepatitis and Blood Safety Department, Public Health England, London, United Kingdom; ${ }^{2}$ Respiratory \& Vaccine Preventable Bac- \\ teria Reference Unit, Public Health England, London, United Kingdom
}

Background and Aims: In April 2010 the 7-valent pneumococcal conjugate vaccine (PCV7) was replaced by the 13-valent PCV. We investigated pneumococcal carriage in children eligible for PCV7 or PCV13 and their household contacts.

Methods: Eligible families in Hertfordshire and Gloucester were identified and a nasopharyngeal swab obtained from consenting household members between July 2012 and March 2013. Samples were cultured for Streptococcus pneumoniae and serotyped by standard methods. For each serotype the ratio of its prevalence in invasive pneumococcal disease (IPD) to its carriage prevalence (case:carrier ratio, CCR) was calculated. Results were compared with previous carriage studies in 2001/2 and 2008/9, before and after PCV7 introduction.

Results: 217 households were included. Among <5 year-olds 47.7\% (95\% confidence interval $41.8-53.5)$ were carrying a pneumococcus compared with $51.0 \%$ in $2008 / 9$ and $48.4 \%$ in $2001 / 2$. The odds of carrying a PCV7 serotype was significantly reduced in $2008 / 9$ and $2012 / 3$ relative to $2001 / 2$, while the odds of carrying any of the extra six PCV13 serotypes increased after PCV7 introduction but declined significantly after PCV13 introduction. The CCRs for the frequently-carried serotypes were relatively low, with the highest CCR observed for serotypes 7F, 19A, 3,8 , and 33F. Across the three carriage studies, CCR estimates were stable for nearly all serotypes.

Conclusion: Carriage of PCV13-only serotypes has rapidly reduced post-PCV13 introduction in both vaccinated and unvaccinated individuals with a continued decline in transmission of PCV7 serotypes. Carriage rates in children remain unchanged, but the low CCRs of replacing serotypes should further reduce overall IPD across all age groups.

No conflict of interest 


\title{
Poster Abstracts
}

ISPPD-0200

Pneumococcal Colonization and Carriage

\section{DIVERSITY OF PNEUMOCOCCAL ISOLATES IN CARRIAGE AND CAUSING MENINGITIS IN PAPUA NEW GUINEA}

\author{
C. Aho $^{1}$, J.P. Dangy², A. Lamelas ${ }^{2}$, A.R. Greenhill ${ }^{3}$, W.S. Pomat ${ }^{1}$, D. Lehmann ${ }^{4}$, G. Pluschke ${ }^{2}$ \\ ${ }^{1}$ Infection and Immunity, Papua New Guinea Institute of Medical Research, Goroka, Papua New Guinea; ${ }^{2}$ Molecular Parasitology and Infection Bi- \\ ology, Swiss Tropical and Public Health Institute, Basel, Switzerland; ${ }^{3}$ School of Applied Sciences and Engineering, Monash University Gipps- \\ land campus, Melbourne, Australia; ${ }^{4}$ Infectious Disease Epidemiology, Telethon Institute of Child Health Research, Perth, Australia
}

Background: Meningitis continues to be associated with high mortality. In Papua New Guinea (PNG), Streptococcus pneumoniae is now the most important cause of meningitis. Where introduced, pneumococcal conjugate vaccines have helped to reduce disease burden but due to limited serotype coverage these vaccines do not protect against all pneumococcal lineages and may contribute to serotype replacement. Serotype-independent protein-based vaccines incorporating several pneumococcal proteins present in all pneumococcal strains may be more favourable. Methods: Focusing on serotypes 2, 5 and 7 (common causes of meningitis in PNG), we characterised 40 invasive pneumococcal isolates from meningitis cases and 22 carriage isolates from PNG to identify dominating clonal complexes.

Results: Multi-locus sequence typing (MLST) from 3 main serotype clusters revealed sequence types (STs) common in Eastern Europe, suggesting importation of these lineages. PNG pspA types correlated with the serotypes. Serotype 2, 5 and 7B/C were associated with the $p s p A$ haplotypes 7, 1 and 11, respectively. No sequence variation within the $p s p A$ sequences of a clonal complex was observed. Broad cross-reactivity was observed when 9 pneumococcal isolates from PNG with different $p s p A$ types were tested for binding with monoclonal antibodies specific for semiconserved $p s p A$ sequences. 9/9 and 7/9 strains were recognized by PspA and Stkp-specific monoclonal antibodies, respectively.

Conclusion: We have identified dominating clonal complexes with limited genetic diversity within the pneumococcal population of colonisation and disease isolates in the Eastern Highlands of PNG. More studies incorporating MLST and pspA typing are needed to shed light on the country-wide diversity of pneumococci in PNG.

No conflict of interest

ISPPD-0163

Pneumococcal Colonization and Carriage

\section{THE PNEUMOEL PROJECT: PNEUMOCOCCAL CARRIAGE AMONG THE ELDERLY LIVING IN PORTUGAL, FINAL RESULTS}

\author{
S.T. Almeida' ${ }^{1}$, S. Nunes ${ }^{1}$, A.S. Paulo ${ }^{1}$, I. Valadares ${ }^{2}$, S. Martins ${ }^{2}$, F. Breia ${ }^{3}$, A. Brito-Avô ${ }^{4}$, A. Morais ${ }^{2}$, H. de Lencastre ${ }^{5}$ \\ ${ }^{1}$ Laboratory of Molecular Microbiology of Human Pathogens, Instituto de Tecnologia Química e Biológica António Xavier, Oeiras, Portugal; ${ }^{2}$ Agru- \\ pamento de Centros de Saúde, Oeiras - Carnaxide, Oeiras, Portugal; ${ }^{3}$ Agrupamento de Centros de Saúde, do Alentejo Central II, Montemor-o- \\ Novo, Portugal; ${ }^{4}$ Private Pediatric Clinic, Private Pediatric Clinic, Lisboa, Portugal; ${ }^{5}$ Laboratory of Microbiology and Infectious Diseases, The Rock- \\ efeller University, New York, USA
}

Background and Aims: While there are several studies addressing pneumococcal colonization of the upper respiratory tract in young children, less is known about carriage in the elderly. This work aimed to evaluate the patterns of Streptococcus pneumoniae carriage in adults over 60 years of age in Portugal.

Methods: Between April 2010 and December 2012, nasopharyngeal and oropharyngeal swabs were obtained from adults over 60 years of age, living in an urban area $(n=1,945)$ or in a rural area $(n=1,416)$. Pneumococci were isolated by routine procedures, serotyped, genotyped (MLST), and antibiotyped. Associations between carriage, socio-demographic and clinical data were analyzed by multiple logistic regression.

Results: Carriage was higher in the rural area than in the urban area (3.4\% vs $1.4 \%$, respectively, $p<0.001)$. Overall, the carriage rate was $2.3 \%$. Multiple logistic regression identified as risk factors for pneumococcal carriage, living in the rural area $(\mathrm{OR}=2.0,95 \% \mathrm{Cl}: 1.2-3.5)$, being at a nursing home $(\mathrm{OR}=2.0,95 \% \mathrm{Cl}: 1.1-3.6)$ and smoking $(\mathrm{OR}=4.4$, $95 \% \mathrm{Cl}: 1.9-9.2)$. In total, 77 pneumococci were isolated, representing 26 serotypes and 40 sequence types (STs). The most prevalent serotypes were 19A (13.0\%), 6C (9.1\%), 22F (9.1\%), 23A (9.1\%), and 35F (7.8\%). Most isolates (93.5\%) had previously described STs. Non-susceptibility to penicillin was found in $11.7 \%$ of the isolates and resistance to macrolides in $19.5 \% ; 15.6 \%$ of all isolates were multidrug resistant.

Conclusion: Pneumococcal carriage in the elderly is low and there is a high serotype and genotype diversity. Living in the rural area, being at a nursing home and smoking were risk factors for pneumococcal colonization.

No conflict of interest 
ISPPD-0559

Pneumococcal Colonization and Carriage

\section{ASSOCIATION BETWEEN BASELINE PLASMA HIV VIRAL LOAD AND NASOPHARYNGEAL PNEUMOCOCCAL COLONIZATION IN CHILDREN WITH HIV INFECTION}

D. Robert ${ }^{1}$, B. Arya $^{1}$, S. Das Bhattacharya ${ }^{1}$, S. Bhattacharyya ${ }^{2}$, S. Mandal ${ }^{3}$, S. Niyogi ${ }^{3}$, R. Das ${ }^{1}$

${ }^{1}$ School of Medical Science and Technology, Indian Institute of Technology-Kharagpur, Kharagpur, India; ${ }^{2}$ Pediatrics, Midnapore Medical College Midnapore (Currently at Medical College Kolkata), Kolkata, India; ${ }^{3}$ Microbiology, NICED Kolkata, Kolkata, India

Background: In developing countries like India, HIV infected children access antiretrovirals late, when their CD4 count drops $<350$. HIV viral load, not CD4 count may be more accurate in predicting risk of NP colonization and IPD in these children.

Objective: To determine the association between HIV viral load and pneumococcal colonization.

Methods: NP swabs were taken at 8 week intervals 4 times across 6 months from HIV infected children in a pneumonia prevention study. Information on age, ART status, CD4 count, viral load, and weight for age z-score was collected in pneumococcal vaccine naïve HIV infected children.

Results: Out of 47 children, 16 had no pneumococcal colonization over six months (Group-1), 31 (66\%) had at least one episode of colonization (Group-2). $9 / 47$ (19\%) of children were in ART, 5 in Group-1 and 4 in Group-2. The mean age of children in both groups was 5. Mean CD4 count was comparable 684 vs 703 in Group-1 and Group-2 ( $p=$ 0.89). Mean waz-score was -2.9 vs. $-3.09(p=0.71)$. Children in Group-2 had a mean viral load of 153,515 almost twice that of Group-1, 80,050. Two children had viral loads of $1 \times 10^{6}$ in Group-2 whereas the highest viral load in Group-1 was $4 \times 10^{5}$.

Conclusion: HIV infected children not qualifying for ART but with high viral loads have increased risk of colonization with pneumococcus. Access to pneumococcal vaccines for this high risk group in developing countries is important.

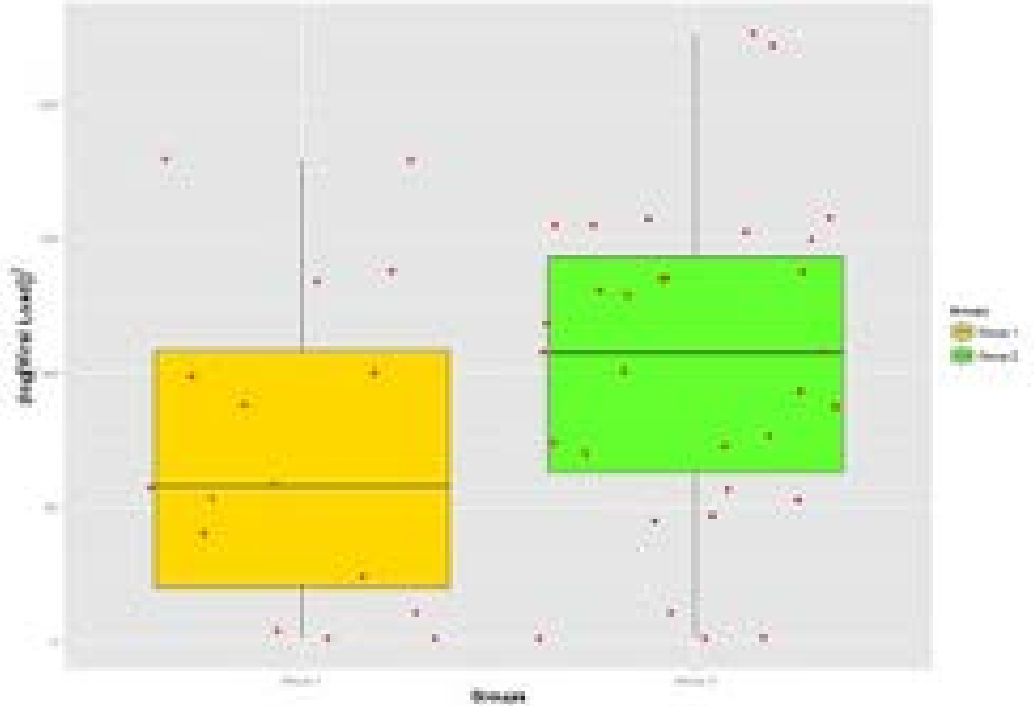

No conflict of interest

ISPPD-0274

Pneumococcal Colonization and Carriage

\section{PNEUMOCOCCAL CARRIAGE IN HIV INFECTED MOTHERS AND CHILDREN IN WEST BENGAL}

B. Arya ${ }^{1}$, S. Das Bhattacharya ${ }^{1}$, S. Niyogi ${ }^{2}$, S. Bhattacharyya ${ }^{3}$, S. Mandal ${ }^{4}$, R. Saurav Das ${ }^{5}$

${ }^{1}$ School of Medical Science and Technology, Indian Institute of Technology-Kharagpur, Kharagpur, India; ${ }^{2}$ Microbiology, National Institute of Cholera and Enteric Diseases Kolkata, Kolkata, India; ${ }^{3}$ Pediatrics, Midnapore Medical College Midnapore (Currently at Medical College Kolkata), Kolkata, India; ${ }^{4}$ Microbiology, National Institute of Cholera and Enteric Diseases Kolkata, Kolkata, India; ${ }^{5}$ School of Medical Science and Technology, Indian Institute of Technology-Kharagpur, Kolkata, India

Background: HIV infected adults and children are at disproportionately high risk of invasive disease from Streptococcus pneumoniae. Pneumococcal conjugate vaccines (PCVs) are effective in HIV infected individuals but are not yet available in government programs.

Objective: To determine baseline carriage of Streptococcus pneumoniae in vaccine naïve mother child pairs affected by HIV, and dynamics of transmission over the course of six months.

Methods: We are conducting an interventional study on the effect of the pneumococcal conjugate vaccine, PCV13 , and the Hib conjugate vaccine on nasopharyngeal carriage, of these vaccine preventable pathogens, in families affected by HIV, in West Bengal. Four nasopharyngeal samples were collected from pneumococcal vaccine naïve, HIV infected children ages 2-15 years, and their mothers, at two month intervals over the course of six months. Calcium alginate swabs were collected and placed in STGG media and processed in the microbiology laboratory using standardized protocols. 
Results: 404 nasopharyngeal swabs have been collected from HIV infected children and 375 swabs from their mothers. In children 100/404 (24.7\%) swabs grew pneumococcus and 19/375 (5\%) swabs in adults were positive for pneumococcus. Fifty children have completed six months of follow-up. In this group, 35/50 (70\%) children had at least one out of four swabs grow pneumococcus over the course of six months. Initial results show that in $31 / 50$ children (62\%), pneumococcal carriage disappeared within 2 months. In 4/50 (8\%), carriage persisted for $>2$ months, and in one case for $>4$ months.

No conflict of interest

ISPPD-0510

Pneumococcal Colonization and Carriage

\section{PNEUMOCOCCAL NASOPHARYNGEAL COLONIZATION DENSITY RELATED TO ADMISSION IMMUNOGLOBULIN TITRES AGAINST THE PATIENT'S OWN SEROTYPE IN PNEUMOCOCCAL PNEUMONIA}

S. Athlin ${ }^{1}$, H. Alpkvist ${ }^{2}$, B. Herrmann ${ }^{3}$, G. Abdeldaim ${ }^{3}$, H.C. Slotved ${ }^{4}$, K. Strålin ${ }^{2}$

${ }^{1}$ Department of Infectious Diseases, Örebro University Hospital, Örebro, Sweden; ${ }^{2}$ Department of Infectious Diseases, Karolinska University Hospital, Stockholm, Sweden; ${ }^{3}$ Department of Clinical Microbiology, Uppsala University Hospital, Uppsala, Sweden; ${ }^{4}$ Department of Microbiology and Infection Control, Statens Serum Institut, Copenhagen, Denmark

Background: Pneumococcal colonization in nasopharynx (NP) is known to precede pneumonia, and NP density is suggested to correlate with disease severity. We studied the correlation between pneumococcal DNA density in NP, and anti-CPS immunoglobulins (Ig) levels to homologous serotypes, in adult community-acquired pneumonia (CAP). Serotypes were grouped according to case fatality rates (CFR; Weinberger, 2004).

Methods: In a prospective study of 235 adult CAP patients, admission sera were collected, and culture on blood, sputum, NP-aspirate (NpA) and NP-swab were performed. Quantitative PCR on NpA for pneumococcal DNA (Spn9802) densities (log-10 DNA copies/mL) were performed. Anti-CPS Ig-titres to homologous serotypes were measured by optical density and compared (\%OD) with pooled sera from post-vaccinated adults. The vaccination frequency was very low.

Results: Of 68 CAP patients with NP pneumococcal DNA, 47 patients with culture-positive pneumococcal aetiology had admission Ig-titres determined. No overall correlation between colonization rates and Ig-titres was noted (table). In cases with medium/low CFR serotypes, colonization densities were significantly higher in those with high, than low, Ig-titres. No difference in duration from onset of illness to admission was noted between serotype categories.

Conclusion: In pneumococcal CAP, high colonization densities and high admission Ig-titres correlated well for serotypes associated with medium/low CFR. Thus, a significant bacterial load in NP may be necessary for pneumonia development, in patients colonized with these serotypes.

\begin{tabular}{|l|l|l|l|l|}
\hline \multirow{2}{*}{ Serotype categories } & \multicolumn{3}{|c|}{ Mean log-10 copies/mL (standard deviation) } & \multirow{2}{*}{$p$ value } \\
\cline { 2 - 5 } & All cases & Low Ig-titres & & \\
\hline All patients $(n=47)^{1}$ & $6.79(1.41)$ & $6.57(1.42)$ & $7.04(1.39)$ & High Ig-titres ${ }^{5}$ \\
\hline High CFR $(n=21)$ & $6.88(1.08)$ & $6.99(0.91)$ & $6.69(1.37)$ & $\mathrm{ns}$ \\
\hline Medium/low CFR ${ }^{3}(n=21)$ & $7.01(1.03)$ & $6.36(1.21)$ & $7.50(0.52)$ & 0.008 \\
\hline
\end{tabular}

${ }^{1}$ Five cases not classified according to CFR; ${ }^{2}<50 \%$ OD; ${ }^{3}>50 \%$ OD

No conflict of interest

ISPPD-0380

Pneumococcal Colonization and Carriage

\section{REFERENCE IDENTIFICATION OF PNEUMOCOCCI ISOLATED FROM NASOPHARYNGEAL SWAB CULTURES OF CHILDREN}

F. Aziz ${ }^{1}$, A. Hotwani ${ }^{1}$, S. Shakoor ${ }^{1}$, F. Jehan ${ }^{1}$, I. Nisar ${ }^{1}$, T. Akhund ${ }^{1}$, S. Qureshi ${ }^{1}$, A. Zaidi ${ }^{1}$

${ }^{1}$ Paediatrics and Child Health, Aga Khan University, Karachi, Pakistan

Background: Optochin (OP) and bile solubility tests (BST) have good specificity when applied to isolates from clinical specimens which have a high pretest probability of identification of pneumococci. The same tests may have different analytical specificity for nasopharyngeal isolates as the upper respiratory tract is a reservoir for both pneumococci and non-pneumococcal streptococci. Non-pneumococcal confounding of OP and BST is known. Objectives: We applied lytA PCR and sequencing to nasophayngeal isolates of 'pneumococci' as reference methods for identification from nasophayngeal swab cultures.

Methods: Nasopharyngeal swabs were collected from well children 3 months to 5Y of age from Karachi, Pakistan as part of a pneumococcal carriage study to evaluate PCV-10 impact. Swabs were collected in STGG media and subcultured on Sheep Blood Agar (SBA). Isolates with suggestive colony morphology (draughtsman or mucoid, alpha hemolytic) were selected for OP and BST. 40 pneumococcal isolates were selected for study on the basis of difficulty in identification (small OP zones or discrepancy in OP and BST). Pneumococcal autolysin gene lytA was detected 
by PCR, followed by sequencing (Macrogen Inc.) as reference identification methods. BLAST analysis by appending sequences to NCBI database using ClustalW2 is ongoing.

Results: Of 40 isolates selected, 12 isolates had discrepant OP and BST results (OP resistant but bile-soluble). lytA was detected in 29 of 40 isolates (72.5\%). Results from sequencing of the PCR product are under evaluation by BLAST analysis.

Conclusion: Results obtained indicate that suggestive colony morphology, OP and BST used together may still confound pneumococcal identification from nasopharyngeal samples.

No conflict of interest

ISPPD-0291

Pneumococcal Colonization and Carriage

\title{
PNEUMOCOCCAL SEROTYPES ISOLATED IN NASOPHARYNGEAL SAMPLES FROM VACCINE NAÏVE HIV INFECTED MOTHERS AND CHILDREN
}

\author{
B. Arya $^{1}$, S. Das Bhattacharya ${ }^{1}$, S. Niyogi ${ }^{2}$, S. Bhattacharyya ${ }^{3}$, R. Jalagandeeswaran ${ }^{4}$, A. Manoharan ${ }^{4}$, S. Mandal ${ }^{2}$, \\ R. Saurav Das ${ }^{1}$, A. Banuja ${ }^{1}$ \\ ${ }^{1}$ School of Medical Science and Technology, Indian Institute of Technology-Kharagpur, Kharagpur, India; ${ }^{2}$ Microbiology, National Institute of Chol- \\ era and Enteric Diseases Kolkata, Kolkata, India; ${ }^{3}$ Paediatrics, Midnapore Medical College Midnapore (Currently at Medical College Kolkata), \\ Kolkata, India; ${ }^{4}$ Medicine I \& ID, Christian Medical College Vellore, Vellore, India
}

Background: Due to the significantly increased risk of invasive disease, HIV infected adults and children are an important group to access pneumococcal conjugate vaccines. Nasopharyngeal colonization precedes invasive disease; determining the pneumococcal serotypes colonizing the nasopharynx of HIV infected individuals is important in the design of new pneumococcal vaccines.

Objective: To determine pneumococcal serotypes from nasopharyngeal samples in vaccine naïve HIV infected mothers and children.

Methods: We are conducting an interventional study looking at the effect of the pneumococcal conjugate vaccine, PCV-13, on nasopharyngeal carriage in families affected by HIV in West Bengal. Here we report baseline serotypes from 43 isolates taken from vaccine naive mothers and children infected with HIV. Samples were collected using calcium alginate swabs and stored in STGG media. These were processed using standard microbiologic methods and pneumococcal isolates were then serotyped at a reference laboratory.

Results: Serotype results for 43 isolates from 35 children and 8 mothers are available. 4 out of 8 strains in mothers are vaccine represented strains- $3,19 \mathrm{~A}, 4$. Three strains in mothers are not vaccine represented-13, 6C, 34. In children 12 out of 35 serotypes are vaccine represented--14, 19A, 19F, 3, 6A, 6B, 9V. The most common serotype was $19 A(5 / 43)$ and $13(6 / 43) .16 / 43(37 \%)$ of isolates are represented in PCV-13. Serotypes that are not vaccine represented include- 10A, 11, 11A, 12, 15A, 15C, 18A, 20, 24F, 29, 31, 36, 38 (one each), 34 (2 isolates), 6C (3 isolates), and 13 (6 isolates).

No conflict of interest

ISPPD-0383

Pneumococcal Colonization and Carriage

\section{DYNAMICS OF MICROBIOTA PROFILES DOMINATED BY STREPTOCOCCUS PNEUMONIAE OVER TIME}

\author{
G. Biesbroek ${ }^{1}$, E. Tsivtsivadze ${ }^{2}$, B.J.F. Keijser ${ }^{2}$, X. Wang ${ }^{1}$, R.H. Veenhoven ${ }^{3}$, E.A.M. Sanders ${ }^{1}$, D. Bogaert ${ }^{1}$ \\ ${ }^{1}$ Pediatric Immunology and Infectious Diseases, UMC Utrecht, Utrecht, Netherlands; ${ }^{2}$ Research Group Microbiology and Systems Biology, \\ TNO Earth Environment and Life Sciences, Zeist, Netherlands; ${ }^{3}$ Research Centre Linnaeus Institute, Spaarne Hospital, Hoofddorp, Netherlands
}

Background and Aims: Insight into bacterial colonization patterns of potential pathogens and commensals in the nasopharynx might elucidate healthy and/or susceptible conditions for development of respiratory disease. We therefore studied the dynamics of microbiota profiles over time in young children.

Methods: We characterized the consecutive nasopharyngeal microbiota profiles of 60 healthy children at the ages of 6 weeks, and 6,12 and 24 months by 16 S GS-flx-titanium-pyrosequencing, and analyzed the consecutive profiles by spectral co-regularized clustering and biomarker detection algorithms.

Results: Overall, we identified 6 distinct microbiota profiles represented by the dominant genera Moraxella, Haemophilus, Streptococcus, or Staphylococcus, a combination of Dolosigranulum and Corynebacterium, plus cluster-specific low abundant biomarker bacteria. We observed specific patterns of change over time, with more stable patterns marked by early presence and high abundance of the Moraxella and Corynebacterium/ Dolosigranulum clusters, and less stable profiles marked by high abundance of the Haemophilus or Streptococcusdominated clusters. The Streptococcus-dominated profile was additionally shaped by high abundance of Prevotella, Gemella, Bacteroidetes (unclassified), Acinetobacter and Porphyromonas, and observed in on average $15 \%$ of the samples, decreasing from $20 \%$ in 6 -week-olds to $7 \%$ in 24 -months-olds. Together with the Haemophilus-dominated profile, the Streptococcus-dominated profile was associated with increased frequency of parent-reported respiratory infections.

Conclusion: The current study enabled us to gain insight in the dynamic nature of nasoparyngeal microbiota in infants. Our results suggest that the composition of early-life microbiota is associated with long-term stability and may predict susceptibility to disease.

No conflict of interest 
ISPPD-0347

Pneumococcal Colonization and Carriage

\title{
LONG-TERM IMPACT OF 23VPPS ON CARRIAGE IN CHILDREN FOLLOWING A REDUCED DOSE PCV PRIMARY SERIES IN INFANCY
}

\author{
L.K. Boelsen ${ }^{1}$, E.M. Dunne ${ }^{1}$, K. Bright ${ }^{2}$, K. Lamb ${ }^{3}$, L. Tikoduadua ${ }^{4}$, F.M. Russell ${ }^{1}$, E.K. Mulholland ${ }^{1}$, P.V. Licciardi $^{1}$, \\ C. Satzke ${ }^{1}$ \\ ${ }^{1}$ Pneumococcal Research, Murdoch Childrens Research Institute, Parkville, Australia; ${ }^{2}$ Global and Tropical Health, Menzies School of Health Re- \\ search, Darwin, Australia; ${ }^{3}$ Clinical Epidemiology and Biostatistics Unit, Murdoch Childrens Research Institute, Parkville, Australia; ${ }^{4}$ Minis- \\ try of Health, Suva, Fiji
}

Background and Aims: The Fiji Pneumococcal Project (FiPP) evaluated a reduced dose PCV7 primary series in infancy, followed by the 23vPPS at 12 months of age. Children receiving 0 or 1 PCV7 dose were given a catch-up dose at 2 years of age. Immune hyporesponsiveness was observed in children aged 18 months who were given 23vPPS, compared with those who were not. Here we assess the long-term impact of 23vPPS vaccination on carriage rates and densities of pneumococci and other common bacterial species. We also compared differences in carriage of pneumococcal serotypes, and examined differences between the two main ethnic populations.

Methods: Nasopharyngeal swabs $(n=194)$ were collected from a subset of healthy FiPP participants now 5-7 years old. Pneumococci were identified by standard culture-based methods and serotyped by latex agglutination/ Quellung. Carriage rates and densities of pneumococci, Haemophilus influenzae, Staphylococcus aureus and Moraxella catarrhalis were determined by quantitative PCR.

Results: Preliminary analysis found there were no differences in pneumococcal carriage rates (40 vs. $52 \%$ ) or vaccine-type carriage ( 20 vs. $17 \%$ ) for 23 vPPS recipients compared with non-recipients. Similar results were found for pneumococcal densities. Analysis of the impact of 23vPPS vaccination on carriage of other species is ongoing. Indigenous Fijian children had significantly higher carriage rates of pneumococci (57 vs. $14 \%$ ), $H$. influenzae (63 vs. $24 \%$ ) and M. catarrhalis ( 86 vs. $41 \%$ ), and lower carriage of S. aureus (23 vs. $41 \%$ ) compared to Indo-Fijian children. Conclusion: Initial analysis shows 23vPPS receipt at 12 months has no long-term impact on pneumococcal carriage rate or density.

No conflict of interest

ISPPD-0406

Pneumococcal Colonization and Carriage

\section{EFFECT OF REPLACING PCV-7 WITH PCV-13 IN THE EXPANDED PROGRAM OF IMMUNIZATION ON NASOPHARYNGEAL CARRIAGE OF PNEUMOCOCCI IN THE GAMBIA}

A. Roca ${ }^{1}$, A. Bojang ${ }^{2}$, C. Bottomley ${ }^{3}$, J. Adetifa ${ }^{2}$, E. Foster-Nyarko², U. Egere ${ }^{2}$, C. Oluwalana ${ }^{1}$, M. Antonio ${ }^{2}$, B. M. Greenwood $^{4}$, B. Kampmann ${ }^{2}$

${ }^{1}$ Disease Control \& Elimination, Medical Research Council Unit The Gambia, Fajara, Gambia; ${ }^{2}$ Vaccinology, Medical Research Coun-

cil Unit The Gambia, Fajara, Gambia; ${ }^{3}$ Tropical Epidemiology Group, London School of Hygiene \& Tropical Medicine, London, United Kingdom;

${ }^{4}$ Faculty of Infectious \& Tropical Diseases, London School of Hygiene \& Tropical Medicine, London, United Kingdom

Background: In July 2011 the Expanded Program of Immunization (EPI) program of The Gambia replaced PVC7 with PCV13. We used this opportunity to determine the impact of replacing PCV7 with PCV13 on the prevalence of pneumococcal nasopharyngeal carriage.

Methods: Healthy Gambian infants who had received 3 doses of a PCV were recruited. Nasopharyngeal swabs were collected from infants and their mothers during cross-sectional surveys undertaken in April-June 2011 (PCV7 group) and in April-June 2012 (PCV13 group). Pneumococci were isolated following standardized methods and serotyped by latex agglutination.

Results: 339 and 350 mother/infants pairs were recruited in the PCV7 and PCV13 vaccinated groups respectively. The overall pneumococcal carriage rate in infants was similar in the two groups $(85.8 \%$ and $84.3 \%, p=0.902)$. Carriage of pneumococci of PCV7 and PCV13 vaccine types (VT) was lower in the PCV13 than in the PCV7 vaccine recipients [4.9\% versus $9.4 \%(p=0.025)$ for PCV7-VT and $18.0 \%$ versus $33.3 .0 \%(p<0.001)$ for PCV13-VT, respectively]. The prevalence of carriage of the six additional serotypes included in the PCV13 and not in the PCV7 vaccine was $13.4 \%$ in the PCV13 and $23.9 \%$ in the PCV7 recipients respectively $(p=0.001)$. However, there was a significant increase in non-typable pneumococci among PCV13 compared to PCV7 vaccinated children $(0.6 \%$ versus $6.6 \%, p=0.005)$. Prevalence of carriage in mothers was similar in both surveys for all study endpoints. Conclusion: Replacing PCV7 by PCV13 decreased the prevalence of pneumococcal carriage of vaccine type pneumococci in vaccinated Gambian infants one year after introduction of PCV13 but not in their mothers

No conflict of interest 


\title{
A NEW BALANCE IN CARRIAGE OF NON-VACCINE PNEUMOCOCCAL SEROTYPES 6.5 YEARS AFTER PCV7 IMPLEMENTATION
}

\begin{abstract}
A.A.T.M. Bosch ${ }^{1}$, R.H. Veenhoven ${ }^{2}$, J.P. Bruinn ${ }^{3}$, A.J. Wijmenga-Monsuur ${ }^{4}$, K. Trzcinski ${ }^{1}$, A. Wyllie ${ }^{1}$, N.Y. Rots ${ }^{4}$, E.A.M. Sanders ${ }^{1}$

${ }^{1}$ Department of Pediatric Immunology and Infectious Diseases, Wilhelmina Children's Hospital University Medical Center Utrecht, Utrecht, Netherlands; ${ }^{2}$ Research Center Linnaeus Institute, Spaarne Hospital and Kennemer Gasthuis, Hoofddorp and Haarlem, Netherlands; ${ }^{3}$ Regional Laboratory of, Public Health, Haarlem, Netherlands; ${ }^{4}$ Center for Immunology of Infectious Diseases and Vaccines, National Institute of Public Health and the Environment, Bilthoven, Netherlands
\end{abstract}

Background: 7-valent pneumococcal vaccine (PCV7) was implemented for infants in the Dutch National Immunization Program (NIP) in 2006, and replaced by PCV10 in 2011. Eradication of vaccine serotype from carriage and emergence of non-vaccine serotypes was observed, with particularly high peaks in serotype 19A carriage 4.5 years after PCV7-implementation(Spijkerman,2012). This suggests a vaccine-induced disbalance among serotypes circulating in children.

Methods: We performed a cross-sectional study 6.5 years after PCV-implementation (2012/13), studying pneumococcal carriage in 330 PCV10-vaccinated 11-month-old children and 330 PCV7-vaccinated 24-month-old children. Present results were compared with carriage data in identical age groups from before, and from 3 and 4.5 years after PCV7-implementation ( $n \approx 330$ /group). Bootstrap calculations were performed to investigate changes in diversity of serotypes carried (Hanage,2010).

Results: In 2012/13, carriage of vaccine-type pneumococci had almost completely disappeared, but overall pneumococcal carriage was similar to the pre-vaccination carriage rates. After 6.5 years, distribution of nonvaccine serotypes was more similar to pre-PCV7 data, indicating a more evenly distributed ranking, and no serotype was exceeding a carriage rate of 10\%. Between 2010 and 2012/13, the high carriage rates of serotype 19A had declined from $12 \%$ to $9 \%$ in 11 -month-old children and from $14 \%$ to $8 \%$ in 24 -month-old children.

Conclusion: Our data suggest a new balance in non-vaccine serotype carriage emerging in vaccinated children 6.5 years after the first PCV implementation in the Dutch NIP. Carriage of serotype 19A declined more prominently in PCV7 vaccinated children and not in PCV10 vaccinated children, and is more likely to a newly achieved balance rather than cross-protection by PCV10.

No conflict of interest

ISPPD-0214

Pneumococcal Colonization and Carriage

\section{STREPTOCOCCUS PNEUMONIAE SEPSIS IN NEWBORNS ASSOCIATED WITH VERTICAL TRANSMISSION: DESCRIPTION OF TWO CASES}

A. Cane ${ }^{1}$, C. Capelli ${ }^{1}$, E. Casanueva ${ }^{1}$, M. Rocca Rivarola ${ }^{1}$, G. Musante ${ }^{1}$, V. Vilches ${ }^{2}$, R. Depardo $^{3}$, S. Fosatti ${ }^{4}$, P. Gaggetti $^{4}$, A. Corso ${ }^{4}$

${ }^{1}$ Pediatrics, Hospital Austral, Ciudad Autónoma de Buenos Aire, Argentina; ${ }^{2}$ Microbiology, Hospital Austral, Ciudad Autónoma de Buenos Aire Argentina; ${ }^{3}$ Pediatrics, Hospital Municipal, Moron, Argentina; ${ }^{4}$ Inei, Anlis, Ciudad Autonoma de Buenos Alresh, Argentina

Background and Aims: Streptococcus pneumoniae (Spn) is an important pathogen, however, neonatal infections due to this organism are rare. Here we report two cases of early-onset pneumococcal septicemia with documented carriage of Spn in the mother's genital tract.

\begin{tabular}{|l|l|l|}
\hline & Case 1 & Case 2 \\
\hline Premature rupture membranes & No & Yes \\
\hline Newborn sign and symptoms & Sepsis-Meningitis & Sepsis \\
\hline Maternal symptoms & No & No \\
\hline Spn isolated in newborns & CSF and Blood & Blood \\
\hline Spn isolated in mothers & Cervix- Vagina & Ovular membranes \\
\hline Spn serotype & 5 & 3 \\
\hline Spn PFGE profile: newborn and mother & A & B \\
\hline Treatment & Cefotaxime & Cefotaxime \\
\hline
\end{tabular}

Methods and Results: Newborns (case 1: 39 weeks; case 2: 32 weeks) presented neonatal sepsis in the first 36 hours of life. Isolates from newborns and mothers were serotyped by Quellung reaction. Antimicrobial susceptibility was determined by agar dilution according CLSI and clonal relationship by Smal-PFGE. Isolates were susceptible to all the antibiotics tested. Isolates from newborns and mothers were, in each case, genetically indistinguishable by PFGE. Babies improved slowly with therapy and they were discharged without clinical abnormalities.

Conclusion: These cases demonstrate that although pneumococcal neonatal sepsis is infrequent, the colonization of the maternal genital tract by this pathogen may lead to septicemia in the newborn.

No conflict of interest 
ISPPD-0480

Pneumococcal Colonization and Carriage

\title{
RELATIONSHIP BETWEEN CHILD'S PNEUMOCOCCAL NASOPHARYNGEAL COLONIZATION DENSITY AND PARENTAL COLONIZATION STATUS
}

\author{
E. Cloessner ${ }^{1}$, L. Grant ${ }^{1}$, J. Vidal ${ }^{2}$, S. Chochua ${ }^{2}$, W. Weatherholtz ${ }^{1}$, K. Klugman² ${ }^{2}$, K. O’Brien ${ }^{1}$ \\ ${ }^{1}$ Center for American Indian Health, Johns Hopkins Bloomberg School for Public Health, Baltimore MD, USA; ${ }^{2}$ Emory University, Roll- \\ ins School of Public Health, Atlanta GA, USA
}

Background: Pneumococcal conjugate vaccines (PCVs) reduce vaccine serotype pneumococcal nasopharyngeal (NP) colonization prevalence and density in vaccinated children. Establishing the relationship between the child's colonization density and the colonization status of close contacts may partly explain the mechanism of PCV indirect effects.

Methods: American Indian households participated in a continuous, cross-sectional NP colonization study (January 2010-April 2012). An NP specimen was collected from each participant and pneumococcus isolated by culture. A subset of NP specimens from households comprised of a child $<8$ years of age, mother and father were selected for subsequent testing by lytA quantitative PCR. Pneumcococcal colonization of $>105$ colony forming units (CFU)/mL was categorized as dense. Multilevel logistic regression analysis measured odds of parental colonization by child's density accounting for intra-household correlation.

Results: 223 families were identified for this analysis ( $n=669$ participants); $28 \%$ of participants $(n=189)$ were positive for pneumococcus. Children were more frequently colonized than their parents ( $53 \%$ vs. $16 \% ; p<0.01)$ and more densely colonized than their parents $\left(10^{5.4} \mathrm{vs.} 10^{4.6} \mathrm{CFU} / \mathrm{mL} ; p<0.01\right)$. Adjusting for the study site where subjects were sampled and differences between American Indian tribes, parents living with a densely colonized child had a greater odds of being colonized (odds ratio (OR): $2.6, p<0.01$ ); mothers were not more likely to be colonized than fathers (OR: 1.09, $p=0.3$ ).

Conclusion: Dense pneumococccal colonization of children is associated with a greater likelihood of parental colonization. Reducing strain-specific colonization density among children through PCV could reduce colonization and risk of disease in family members and other close contacts.

No conflict of interest

ISPPD-0316

Pneumococcal Colonization and Carriage

\section{QUANTIFICATION OF NASOPHARYNGEAL STREPTOCOCCUS PNEUMONIAE CARRIAGE AND LOAD IN GAMBIAN NEONATES AND INFANTS}

\author{
E. Coe ${ }^{1}$, B. Kwambana ${ }^{1}$, E. Clarke ${ }^{2}$, G. Weinstock $^{3}$, M. Antonio ${ }^{1}$ \\ ${ }^{1}$ Vaccinology, Medical Research Council Unit The Gambia, Fajara, Gambia; ${ }^{2}$ Infant Immunology, Medical Research Council Unit The Gambia, \\ Fajara, Gambia; ${ }^{3}$ The Genome Institute, Washington University School of Medicine, St. Louis, USA
}

Background and Aims: Nasopharyngeal (NP) carriage of Streptococcus pneumonia is an important risk factor for invasive pneumococcal disease. This study characterizes NP carriage rates and loads of S. pneumoniae in the first 12 weeks of life in a rural cohort in The Gambia.

Methods: 480 NP swabs were collected from 120 infants at monthly intervals from within minutes of birth to 3 months. Quantitative PCR targeting the autolysin gene ( $L y t A)$ was performed on the DNA extracts from the swabs. Results: Preliminary results from 76 newborns showed a significant increase in pneumococcal carriage $(p<0.01)$ with $32 \%$ at birth, $59 \%$ at one month, $68 \%$ at two months and $85 \%$ at three months. The mean pneumococcal load also increased significantly from $1.09 \times 10^{1}$ cells $/ \mathrm{mL}$ at birth, increasing to $1.56 \times 10^{3} \mathrm{cells} / \mathrm{mL}, 1.72 \times 10^{3}$ cells/ $\mathrm{mL}$ and $1.80 \times 10^{4}$ cells $/ \mathrm{mL}$ at one, two and three months respectively, $\left(\mathrm{X}^{2}(3)=84.103, p<0.01\right)$. Logistic regression analysis revealed that birth weight $>3.1 \mathrm{~kg}$ (odds ratio $(\mathrm{OR}) 1.72,95 \% \mathrm{Cl} 1.04-2.84, p=0.03$ ), health centre rather than hospital births (OR $0.38,95 \% \mathrm{Cl} 0.18-0.78, p<0.01)$, and having $1-3$ older siblings (OR $2.1,95 \% \mathrm{Cl} 1.07-4.16, p$ $=0.03)$ all significantly increased pneumococcal carriage. However gender had no significant effect $(\mathrm{OR} 1.21,95 \% \mathrm{Cl}$ $0.75-1.94, p=0.44)$. After adjusting for birth weight, place of birth and number of siblings, only place of birth and having $1-3$ siblings remain significant, $(\mathrm{OR} 0.38,95 \% \mathrm{Cl} 0.18-0.80, p=0.01)$ and $(\mathrm{OR} 2.13,95 \% \mathrm{Cl} 1.00-4.56, p=0.05)$ respectively.

Conclusion: Both pneumococcal carriage and load increase significantly within weeks of birth which could implicate susceptibility to neonatal infections. This data is important for future studies looking at natural pneumococcal protection dynamics in neonates and young infants.

No conflict of interest 
ISPPD-0309

Pneumococcal Colonization and Carriage

\section{PNEUMONIA PREVENTION IN FAMILIES AFFECTED BY HIV: STUDY DESIGN INVESTIGATING INDIRECT BENEFITS OF PNEUMOCOCCAL CONJUGATE VACCINATION IN HIGH RISK HOUSEHOLD}

B. Arya ${ }^{1}$, S. Das Bhattacharya ${ }^{1}$, S. Bhattacharyya ${ }^{2}$, S. Niyogi ${ }^{3}$, S. Panda ${ }^{3}$, R.S. Das ${ }^{1}$

${ }^{1}$ School of Medical Science and Technology, Indian Institute of Technology-Kharagpur, Kharagpur, India; ${ }^{2}$ Pediatrics, Midnapore Medical College Midnapore (Currently at Medical College Kolkata), Kolkata, India; ${ }^{3}$ Microbiology, National Institute of Cholera and Enteric Diseases Kolkata, Kolkata, India

Bacterial pneumonia due to Streptococcus pneumoniae remains the leading cause of hospital admission in HIV infected individuals. Pneumococcal conjugate vaccine (PCV) are effective in HIV infected individuals for the prevention of invasive disease from this pathogens; however, it is not yet available through government programs in India. Studies looking at the impact of these vaccines on nasopharyngeal colonization in families affected by HIV can potentially inform health policy in India. We describe the design for our ongoing study looking at the impact of pneumococcal conjugate vaccine in families affected by HIV being carried out in West Bengal. Organizing a community based clinical study depends much on cultural expectations and socio-political scenario of the sites involved. We describe our experience with different tools used in the consent and follow up process in our study

No conflict of interest

ISPPD-0399

Pneumococcal Colonization and Carriage

\section{MULTI-LOCUS SEQUENCE TYPES OF NASOPHARYNGEAL ISOLATES OF PNEUMOCOCUS FROM NORTHEAST INDIA}

U. Devi ${ }^{1}$, V. Malik ${ }^{1}$, J. Mahanta ${ }^{1}$

${ }^{1}$ Bacteriology, Regional Medical Research Centre North east Region(ICMR), Dibrugarh, India

Background \& Aims: Sequence types prevailing in Northeast India are not known. We present sequence types currently completed.

Methods: DNA was extracted using phenol-chloroform method. Primer pairs used for amplifying the seven housekeeping genes namely $\operatorname{aroE}, g d h, g k i, \operatorname{rec} P$, spi, xpt and $d d l$ were obtained from those listed in MLST website (http://spn.mlst.net/). Amplicons were sequenced on both sides (commercially) and sequences were edited using Genious Pro v 5.4.6. The core sequences were uploaded to pneumococcus database for allele and ST identification. Results: Table below depicts the allele numbers and sequence types.

\begin{tabular}{|l|l|l|l|l|l|l|}
\hline & Isolate 1 & Isolate 2 & Isolate 3 & Isolate 4 & Isolate 5 & Isolate 6 \\
\hline aroE & 16 & 10 & 2 & 2 & 10 & 16 \\
\hline gdh & 9 & 11 & 11 & 11 & 11 & 289 \\
\hline$g k i$ & 111 & 6 & 10 & 10 & 6 & 4 \\
\hline recP & 5 & 1 & 5 & 5 & 1 & 83 \\
\hline spi & 7 & 7 & 6 & 6 & 7 & 15 \\
\hline$x p t$ & 329 & 146 & 129 & 129 & 146 & 20 \\
\hline$d d l$ & 8 & 5 & 17 & 17 & 5 & 168 \\
\hline ST & 6690 & 6030 & 4908 & 4908 & 6030 & 5282 \\
\hline
\end{tabular}

Conclusion: A total number of 4 different ST's have been identified. The ST's obtained are similar to those nasopharyngeal isolates from neighboring countries including Thailand (ST6690), Nepal (ST6030; ST5282). Two of them (ST 4908) are similar to meningeal isolates from India.

No conflict of interest 
ISPPD-0265

Pneumococcal Colonization and Carriage

\title{
INDIRECT EFFECTS OF PCV10 VACCINATION IN YOUNG UNVACCINATED INFANTS IN FIJI
}

\author{
E. Dunne ${ }^{1}$, S. Semi ${ }^{1}$, C.L. Pell ${ }^{1}$, K.S. Stanhope ${ }^{1}$, E. Rafai ${ }^{2}$, K. Bright ${ }^{3}$, B.D. Porter ${ }^{1}$, T. Ratu ${ }^{2}$, M. Cama ${ }^{2}$, M. Kama ${ }^{2}$, \\ K. Gould ${ }^{4}$, J. Hinds ${ }^{4}$, L. Tikoduadua ${ }^{2}$, J. Kado ${ }^{2}$, R. Devi ${ }^{2}$, E.K. Mulholland ${ }^{1}$, C. Satzke ${ }^{1}$, F.M. Russell ${ }^{1}$ \\ ${ }^{1}$ Pneumococcal Research, Murdoch Childrens Research Institute, Parkville, Australia; ${ }^{2}$ Ministry of Health, Suva, Fiji; ${ }^{3}$ Global and Tropical Health, \\ Menizes School of Health Research, Darwin, Australia; ${ }^{4}$ Bacterial Microarray Group, St. George's Univerity of London, London, United Kingdom
}

Background and Aims: Although indirect effects of pneumococcal conjugate vaccination (PCV) have been welldocumented in adults, there are few data regarding the herd effects in young infants. This age group are too young to be vaccinated with PCV but are at high risk for severe bacterial infections. Fiji introduced PCV10 in 2012. The impact of 10valent pneumococcal conjugate vaccine (PCV10) introduction on nasopharyngeal carriage is being monitored as part of the New Vaccine Evaluation Project. The indirect effects of PCV10 introduction will be measured in young infants (5-8 wk) through annual carriage surveys (pre- and post-PCV10 introduction).

Methods: Nasopharyngeal swabs were collected from 500 healthy infants aged 5-8 wk before, and one year after, PCV10 introduction. Swabs were stored in STGG and frozen at $-80^{\circ} \mathrm{C}$ until analysis. Streptococcus pneumoniae, Staphylococcus aureus, and Haemophilus influenzae were detected using quantitative PCR, and pneumococcal molecular serotyping performed by microarray.

Results: Preliminary analysis of the pre-PCV10 swabs found carriage rates of $30 \%$ for S. pneumoniae, $23 \%$ for S. aureus, and $27 \%$ for $H$. influenzae. Serotyping by microarray found that $40 \%$ of pneumococci identified were PCV10 serotypes. We are currently examining swabs taken one year post-PCV10 introduction to investigate potential herd immune effects on nasopharyngeal carriage in this age group.

Conclusion: Our studies in Fiji provide a unique opportunity for investigating PCV impact in young, unvaccinated infants. Data will help to evaluate potential herd effects on carriage of pneumococcal serotypes and other pathogens in young infants in a low-income country setting.

No conflict of interest

ISPPD-0459

Pneumococcal Colonization and Carriage

\section{MICROARRAY SEROTYPING IN THE DETECTION OF LOW ABUNDANCE PNEUMOCOCCAL SEROTYPES WITHIN THE CONTEXT OF A PCV-7 TRIAL}

\author{
C. Ebruke ${ }^{1}$, B. Kwambana ${ }^{1}$, J. Hinds ${ }^{2}$, K.A. Gould ${ }^{2}$, E. Foster-Nyarko ${ }^{1}$, B. Wren ${ }^{3}$, M. Antonio ${ }^{1}$ \\ ${ }^{1}$ Vaccinology, Medical Research Council Unit The Gambia, Fajara, Gambia; ${ }^{2}$ Division of Clinical Sciences, St. George's University of London, Lon- \\ don, United Kingdom; ${ }^{3}$ Department of Pathogen Molecular Biology, London School of Hygiene and Tropical Medicine, London, United Kingdom
}

Background and Aims: Detection of multiple serotypes of Streptococcus pneumoniae is necessary to understand the effects of pneumococcal conjugate vaccination in carriage studies. This study aimed to compare serotyping by latex agglutination and microarray within the context of a 7-valent pneumococcal conjugate vaccine (PCV-7) trial.

Methods: Nasopharyngeal swabs (NPS) were collected from 22 newborns at regular intervals up to 12 months of age. All children received at least three doses of PCV-7. S. pneumoniae was identified by conventional microbiology techniques. S. pneumoniae isolates were serotyped using the latex agglutination and microarray sweep method. Results: Microarray detected 2, 3 and 4 serotypes in $28 \%, 11 \%$ and $3 \%$ of 157 NPS respectively, compared to $7 \%$, $1 \%$ and $0 \%$ by latex agglutination $(p<0.001)$. Although a common serogroup was detected in $94 \%$ of NPS by both methods, latex agglutination was more likely to miss co-carried low abundance serotypes. For instance, in one NPS both methods detected serotype 6A/B, however, latex agglutination did not detect serotypes $37,16 \mathrm{~F}$ and an NT which had relative abundance of $8 \%, 6 \%$ and $3 \%$ by microarray respectively. In four other NPS collected from the same subject at consecutive timepoints, serotype $16 \mathrm{~F}$ was present but appeared to be cleared from carriage by latex agglutination. The predominant serotypes detected by latex agglutination were $19 \mathrm{~A}(10 \%)$ and $13(9 \%)$ while by microarray they were $13(8 \%)$ and $19 \mathrm{~A}(7 \%)$.

Conclusion: We report improved detection of multiple serotypes using microarray method over latex agglutination. Highly sensitive molecular techniques should be employed in post-vaccine implementation carriage studies.

No conflict of interest 
ISPPD-0248

Pneumococcal Colonization and Carriage

\title{
OROPHARYNGEAL AND NASOPHARYNGEAL SAMPLING FOR THE DETECTION OF ADOLESCENT STREPTOCOCCUS PNEUMONIAE CARRIERS
}

\author{
S. Esposito ${ }^{1}$, L. Terranova ${ }^{1}$, A. Zampiero ${ }^{1}$, F. Manzoni ${ }^{1}$, L. Senatore ${ }^{1}$, W. Peves Rios ${ }^{1}$, N. Principi ${ }^{1}$ \\ ${ }^{1}$ Department of Pathophysiology and Transplantation Università degli Studi di Milano, Pediatric Highly Intensive Care Unit Fondazione IRC- \\ CS Ca' Granda Ospedale Maggiore Policlinico, Milan, Italy
}

Background and aims: Monitoring the dynamics of pneumococcal carriage makes it possible to evaluate the epidemiological characteristics of Streptococcus pneumoniae disease and the theoretical coverage offered by pneumococcal vaccines. It has been demonstrated that the nasopharyngeal (NP) sampling of respiratory secretions is superior to oropharyngeal (OP) sampling for identifying pneumococci carried by younger children, but adult data are conflicting and there are no studies of adolescents.

Methods: In order to compare the efficiency of OP and NP sampling in identifying and quantifying S. pneumoniae carriage in healthy adolescents, two swab samples were obtained from 530 adolescents aged $15-19$ years, the first taken from the posterior pharyngeal wall through the mouth (OP) and the second through the nose (NP). Bacterial genomic DNA was tested for the autolysin-A ( LytA) and wzg (cpsA) genes of S. pneumoniae in order to evaluate pneumococcal carrier status. Positive cases were serotyped.

Results: S. pneumoniae was identified in $35.8 \%$ of the OP swabs and $3.5 \%$ of the NP swabs $(p<0.0001)$. The serotypes included in the 13-valent pneumococcal conjugate vaccine (PCV13) were found in all but two OP samples $(98.9 \%)$ and only $64.7 \%$ of the NP samples $(p<0.0001)$. The most frequently identified PCV13 serotype in both groups was $19 \mathrm{~F}$, followed by serotypes 5 and $9 \mathrm{~V}$.

Conclusion: OP sampling appeared significantly more effective than NP sampling in identifying and characterising pneumococcal carrier status in adolescents. This suggests that OP sampling should be used when evaluating the dynamics of pneumococcal carriage among adolescents and the theoretical coverage offered by PCV13.

No conflict of interest

ISPPD-0430

Pneumococcal Colonization and Carriage

\section{PNEUMOCOCCAL CARRIAGE AND ANTIMICROBIAL RESISTANCE IN HIV-INFECTED CHILDREN IN MOZAMBIQUE}

S. Fernando Massora ${ }^{1}$, B. Sigauque ${ }^{1}$, S. Acácio ${ }^{1}$, R. Santos ${ }^{2}$, D. Vubil ${ }^{1}$, F. Pimenta ${ }^{3}$, I. Moura ${ }^{3}$, C. Whitney ${ }^{3}$, E. Macete ${ }^{1}$, P. Alonso ${ }^{4}$, J.R. Verani ${ }^{3}$, M.G. Carvalho ${ }^{3}$

${ }^{1}$ Clinic, Manhiça Health Research Center, Maputo, Mozambique; ${ }^{2}$ Clinic, Hospital Central de Nampula, Maputo, Mozambique; ${ }^{3}$ Clinic, Respiratory Diseases Branch Centers for Disease Control and Prevention, Atlanta, USA; ${ }^{4}$ Clinic, Barcelona Center for International Health Research (CRESIB Hospital Clinic - Universitat de Barcelona), Barcelona, Spain

Background: HIV-infected children are at high risk for pneumococcal disease. Antimicrobial-resistant Streptococcus pneumoniae can lead to poor clinical outcomes and increased cost of medical care. We examined antimicrobial susceptibility among nasopharyngeal pneumococcal isolates obtained from HIV-infected children in Mozambique before the introduction of the 10-valent pneumococcal conjugate vaccine (PCV10) in April 2013.

Methods: Between October 2012 and March 2013, we enrolled HIV-infected children age $<5$ years presenting for routine care at seven HIV clinics in 3 sites, including Maputo (urban-south), Nampula (urban-north), and Manhi?a (rural-south). A single nasopharyngeal swab was obtained and cultured following broth enrichment. Susceptibility to commonly-used antibiotics was evaluated for pneumococcal isolates using broth dilution susceptibility tests according to definitions from the 2013 Clinical Laboratory Standard Institute guidelines. Serotyping was performed using Quellung reaction.

Results: A total of 343 isolates were obtained from 336 HIV-infected children. Overall, 304 (88.6\%) isolates were resistant to co-trimoxazole, $56(16.3 \%)$ to erythromycin, $23(6.7 \%)$ to chloramphenicol, and $1(0.3 \%)$ to amoxicillin. None were resistant to ceftriaxone or penicillin. Three isolates had intermediate resistance to penicillin (MIC=4); all 3 were PCV10 serotypes. The proportion of resistant isolates that were serotypes covered by PCV10 for cotrimoxazole, erythromycin, chloramphenicol and amoxicillin was $51.6 \%, 97.6 \%, 72.7 \%$ and $100 \%$ respectively. Conclusion: Given the low prevalence of $\beta$-lactam resistance, those agents continue to be useful first-line antibiotics for treating pneumococcal disease in HIV-infected children in Mozambique. PCV10 introduction will likely lead to an important decline in the circulation of resistant pneumococci in this population.

No conflict of interest 


\title{
ISPPD-0396
}

Pneumococcal Colonization and Carriage

\section{THE KINETICS AND PHENOTYPE OF THE HUMAN B-CELL RESPONSE TO PNEUMOCOCCAL POLYSACCHARIDE AND PROTEIN ANTIGENS DURING EXPERIMENTAL CARRIAGE}

D.M. Ferreira ${ }^{1}$, S. Pennington ${ }^{1}$, Q. Masood ${ }^{1}$, A.D. Wright ${ }^{2}$, C. Hancock ${ }^{2}$, J. Gritzfeld ${ }^{1}$, E.N. Miyaji ${ }^{3}$, A. Collins ${ }^{1}$, S.B. Gordon $^{1}$

${ }^{1}$ Clinical Sciences, Liverpool School of Tropical Medicine, Liverpool, United Kingdom; ${ }^{2}$ Respiratory, NIHR Royal Liverpool and Broadgreen University Hospitals NHS Trust, Liverpool, United Kingdom; ${ }^{3}$ Biotechnology Center, Butantan Institute, Sao Paulo, Brazil

Background and Aims: We have previously reported that Experimental Human Carriage was an immunizing and protective event. Now, in volunteers inoculated with pneumococcus, we characterize the kinetics of circulating antigen-specific plasma and memory B cell populations in blood and further characterize their phenotype in blood and in the lung (bronchoalveolar lavage (BAL)).

Method: Healthy volunteers were inoculated with a 6B pneumococcal strain. Blood samples were collected at days $0,7,14$ and 35 post inoculation for ELISPOT analyses. Flow cytometric analysis were performed using B cells purified with anti-CD19 microbeads. Cells were labeled for surface phenotype and cellular antigen-specificity determined using biotinylated capsular polysaccharide (PS) 6B labeled with anti-biotin-FITC.

Results: We evaluated IgG and IgA secreting plasma and memory B cells to PS6B, whole cell pneumococcus, PspA, $\mathrm{PspC}$ and Flu in 96 samples collected from 25 volunteers ( 15 carriers and 10 non-carriers). Preliminary analysis revealed a transient increase in the numbers of circulating plasma cells peaking at day 7 for PS6B and at day 14 for PspA. Carriage increased B1a but not B1b cells in blood at day 21 . We are now analyzing the impact of carriage upon $B$ cell populations in the lung.

Conclusion: While B1a cells produce serum antibody, B1b cells are involved in long-term protection against bacterial pathogens such as pneumococcus. Our results suggest that although pneumococcal nasal exposure is immunizing and elicits antibodies, this response may be restricted to the B1a and not the B1b subset. Acknowledgements: The BMGF GCE and MRC-FAPESP

No conflict of interest

ISPPD-0514

Pneumococcal Colonization and Carriage

\section{LIVE ATTENUATED INTRANASAL INFLUENZA VACCINE DOES NOT INCREASE RATES OR DENSITY OF NASAL PNEUMOCOCCAL COLONISATION IN HEALTHY CHILDREN}

\author{
V. Thors ${ }^{1}$, B. Morales-Aza ${ }^{1}$, H. Christensen², B. Vipond ${ }^{3}$, P. Muir ${ }^{3}$, A. Finn ${ }^{1}$ \\ ${ }^{1}$ Cellular and Molecular Medicine, University of Bristol, Bristol, United Kingdom; ${ }^{2}$ Social and Community Medicine, University of Bristol, Bristol, \\ United Kingdom; ${ }^{3}$ South West Regional Virology Laboratory, Public Health England, Bristol, United Kingdom
}

Background and Aims: Respiratory viral infections may affect bacterial colonisation of the human nasopharynx and efficiency of transmission. The particular interaction between influenza and Streptococcus pneumoniae (Sp) has received particular attention. We investigated whether trivalent live attenuated influenza vaccine (LAIV) influences nasal carriage and density of $\mathrm{Sp}$ in healthy children.

Methods: 151 children were recruited into a randomised controlled stepped-wedge study to start LAIV ( 2 doses, one month apart) either at an initial study visit or one month later, with nasopharyngeal swabs taken 7 and 28 days after each dose, allowing comparisons both between vaccinated and unvaccinated children and pre- and postvaccine. Swabs, stored in STGG, were analysed using real-time PCR (RT-PCR) for lytA.

Results: At 7 days post- vaccination S.pneumoniae was detected $(C T<35)$ in $69.2 \%$ children and $66.2 \%$ of controls $(p$ $=0.706$ ) with similar density in the two groups at both day 7 and 28 ( $p=0.50$ and 0.46 , respectively). Likewise, when values pre- and both 7 and 28 days post-first vaccine dose were compared in all children studied, no differences in colonisation rates were shown.

Conclusion: LAIV did not cause detectable changes in rates or density of nasal carriage with pneumococcus, measured by PCR, in this population, among whom colonisation rates were high. It seems possible that while infection with wild type influenza and other respiratory viral infections may affect bacterial colonisation, the largely sub-clinical infection caused by attenuated vaccine viruses do not.

Conflict of interest 
ISPPD-0495

Pneumococcal Colonization and Carriage

\section{NASOPHARYNGEAL PNEUMOCOCCUS IS NEITHER COMMONER NOR MORE ABUNDANT IN ACUTE OTITIS MEDIA WITH SPONTANEOUS OTORRHOEA; OTHER OTOPATHOGENS ARE LESS ABUNDANT}

B. Morales-Aza ${ }^{1}$, P. Sikora-Liszka ${ }^{1}$, L. Januario ${ }^{2}$, F. Rodrigues ${ }^{2}$, A. Finn ${ }^{1}$

${ }^{1}$ Cellular and Molecular Medicine, University of Bristol, Bristol, United Kingdom; ${ }^{2}$ Infectious Diseases Unit \& Emergency Service, Hospital Pediatrico de Coimbra, Coimbra, Portugal

Background and Aims: Studies comparing rates and/or densities of Streptococcus pneumoniae (Sp) colonisation in children with respiratory infections and in health are conflicting. We tested the hypothesis that they are unchanged in acute otitis media with spontaneous otorrhoea (AOMSO).

Methods: In February-March 2011 we swabbed 516 children attending nurseries(AN) and 104 with AOMSO.

Nasopharyngeal swabs were stored at $-80^{\circ} \mathrm{C}$ in STGG broth and $50 \mu$ l cultured using standard techniques. Sp, Haemophilus influenzae ( $\mathrm{Hi}$ ) and Moraxella catarrhalis $(\mathrm{Mc})$ were identified and densities assessed by scoring numbers of colonies (0-5 where 5 is $>100)$.

Results: $80 \%$ of the children with AOMSO attended nurseries. By univariate analysis, rates of colonisation and mean densities did not differ apart from Mc density. By multivariate analysis (adjusting for age), colonisation rates and densities for both $\mathrm{Hi}$ and $\mathrm{Mc}$ were lower in AOMSO. The mean total number of bacterial species identified was similar in the two groups (1.7vs1.8; $p=0.674)$.

Conclusion: Children with AOMSO did not have higher rates or densities of Sp but significantly lower densities of both $\mathrm{Hi}$ and $\mathrm{Mc}$ were seen. This relative imbalance between species in otitis may point to the ecological conditions predisposing to disease.

\begin{tabular}{|l|l|l|l|l|l|l|}
\hline & & Univariate & & & Multivariate & \\
\hline Colonisation & & AOMSO $(n=108)$ & AN $(n=516)$ & $p$ value & OR $(95 \% \mathrm{Cl})$ & $p$ value \\
\hline Rate & $\mathrm{Sp}$ & $58(56 \%)$ & $312(61 \%)$ & 0.419 & $1.15(0.69-1.92)$ & 0.581 \\
\hline & $\mathrm{Hi}$ & $21(20 \%)$ & $88(17 \%)$ & 0.422 & $1.11(0.58-2.01)$ & 0.749 \\
\hline & $\mathrm{Mc}$ & $65(63 \%)$ & $345(67 \%)$ & 0.446 & $1.25(0.72-2.19)$ & 0.418 \\
\hline Density & $\mathrm{Sp}$ & 4.1 & 4.2 & 0.641 & $1.19(0.60-2.34)$ & 0.612 \\
\hline & $\mathrm{Hi}$ & 3.9 & 4.3 & 0.067 & $4.07(1.16-14.25)$ & 0.028 \\
\hline & $\mathrm{Mc}$ & 3.9 & 4.2 & 0.024 & $1.93(1.08-3.45)$ & 0.026 \\
\hline
\end{tabular}

No conflict of interest

ISPPD-0280

Pneumococcal Colonization and Carriage

\section{CHARACTERISATION OF CARRIAGE FROM WHOLE GENOME SEQUENCES TO ASSESS PCV7 IMPACT AND INFORM THE FUTURE OF PNEUMOCOCCAL VACCINATION}

R. Gladstone ${ }^{1}$, J. Jefferies ${ }^{2}$, S. Faust ${ }^{3}$, S. Bentley ${ }^{1}$, S. Clarke ${ }^{4}$

${ }^{1}$ Pathogen Genomics, Wellcome Trust Sanger Institute, Cambridge, United Kingdom; ${ }^{2}$ Academic Unit of Clinical and Experimental Sciences, University of Southampton, Southampton, United Kingdom; ${ }^{3}$ NIHR Wellcome Trust Clinical Research Facility, University Hospital Southampton Foundation NHS Trus, Southampton, United Kingdom; ${ }^{4}$ Institute for Life Sciences, University of Southampton, Southampton, United Kingdom

Pneumococcal conjugate vaccines (PCV) were implemented from 2006 in the UK. Colonisation precedes disease therefore carriage can be an early indicator of epidemiological changes. Circulating pneumococci were highly characterised to assess vaccine impact and generate data informative to future vaccine policy and formulation. Nasopharyngeal swabs were collected from children of four years of age and under each winter from 2006/7 to 2010/11. Pneumococci were isolated using conventional microbiology. Next generation sequencing was utilised to comprehensively type isolates and determine sequence variation in numerous genes important to pneumococcal typing and virulence using a single method. Carriage remained stable. Conversely PCV7 and PCV13-unique vaccine serotypes (VT) significantly decreased with concomitant increases in non-vaccine serotypes (NVT). Significant decreases for VT 6A, 6B, 19F and 23F and increases for NVT 21, 23B, 33F and 35F were detected. Significant changes were observed for associated genotypes. Novel non-synonymous sequence variation was observed to be common in capsular defining genes within serotypes, particularly for serogroups 19 and 6 . Newly described serotypes 6D and 6G sequences were detected. Ten novel pneumolysin protein alleles were observed. Pneumolysin sequences associated with non-pneumococcal streptococci were detected in pneumococci and vice-versa. Serotype replacement resulted in increased NVT colonisation, which could progress to disease cases. Future control of pneumococcal disease through vaccination will require response to replacement, targeting prevalent replacing serotypes or broader acting pneumococcal vaccines. However strategies should account for potential target diversity and consequent specificity. Whole genomes offer an invaluable resource for documenting sequence variability of genes throughout the genome.

Conflict of interest 
ISPPD-0044

Pneumococcal Colonization and Carriage

\title{
INDIRECT EFFECT OF THE 13-VALENT PNUEMOCOCCAL CONJUGATE VACCINE USE IN CHILDREN ON ADULT PNEUMOCOCCAL COLONIZATION AND DISEASE IN ALASKA
}

\author{
P. Gounder ${ }^{1}$, D.J.T. Bruden ${ }^{1}$, M.G. Bruce ${ }^{1}$, K. Rudolph ${ }^{1}$, C. DeByle ${ }^{1}$, A. Reasonover ${ }^{1}$, R.J. Singleton ${ }^{1}$, G. Thompson ${ }^{1}$, \\ M. Toomey ${ }^{1}$, T.W. Hennessy ${ }^{1}$ \\ ${ }^{1}$ Centers for Disease Control, Arctic Investigations Program Office Division of Preparedness and Emerging Infections National Center for Emerg- \\ ing and Zoonotic Infectious Disease, Anchorage, USA
}

Background and Aims: The 13-valent pneumococcal conjugate vaccine (PCV13) replaced the 7-valent pneumococcal conjugate vaccine (PCV7) in the Alaska childhood immunization schedule in 2010. We assessed the indirect effect of PCV13 on pneumococcal colonization and disease in Alaskan adults.

Methods: To determine pneumococcal colonization, we recruited a convenience sample of residents of all ages from 8 rural villages and children aged $<5$ years at 2 urban pediatric clinics annually during 2008-2012; we determined their PCV13 vaccination status and obtained nasopharyngeal swab specimens. Pneumococci were identified/serotyped by standard laboratory methods. We identified invasive pneumococcal disease (IPD) cases, defined as Streptococcus pneumoniae cultured from a sterile site, through conducting statewide surveillance. Results: During 2008-2012, we recruited 4,310 children aged $<5$ years ( $89 \%$ with $>1$ dose PCV13 in 2011-2012) and 8,336 adults aged $>18$ years. Overall pneumococcal carriage among rural and urban children $<5$ years and adults $>18$ years remained stable (mean/year: 66\%, 35\%, and 14\%, respectively). From 2008 through 2012 , PCV13 serotype carriage declined among children $<5$ years $(13 \%-4 \%, p$-value for trend $<0.01)$ and adults $>18$ years $(4 \%-$ $1 \%, p<0.01)$. During $2011-2012,6 / 943(<1 \%)$ adults $>50$ years were colonized by PCV13 serotypes. PCV13-IPD rates among Alaskan adults $>18$ years declined from 8.2/100,000 persons in 2008 to 5.2/100,000 in 2012 ( $p$-value $<0.01$ ). Conclusion: Pediatric PCV13 vaccination provided indirect protection against vaccine-type colonization and IPD to unvaccinated adults. Potential benefits to adults aged $>50$ years from routine PCV13 vaccination appears to be small because PCV13 serotype carriage is low and PCV13-IPD rates are declining due to indirect protection.

Conflict of interest

ISPPD-0160

Pneumococcal Colonization and Carriage

\section{ONTOGENY OF SERUM ANTIBODY RESPONSES TO 27 PNEUMOCOCCAL PROTEIN ANTIGENS OVER THE FIRST 9 MONTHS OF LIFE: A COMPARATIVE STUDY}

\author{
N. Green ${ }^{1}$, P. Turner ${ }^{2}$, J. Ojal ${ }^{3}$, C. Turner ${ }^{2}$, F. Nosten ${ }^{2}$, J.A.G. Scott ${ }^{3}$, D. Goldblatt ${ }^{1}$ \\ ${ }^{1}$ Immunobiology, UCL Institute of Child Health, London, United Kingdom; ${ }^{2}$ Centre for Tropical Medicine, University of Oxford, Oxford, Unit- \\ ed Kingdom; ${ }^{3}$ Centre for Geographic Medicine Research-Coast, KEMRI-Wellcome Trust Research Programme, Kilifi, Kenya
}

Exposure to Streptococcus pneumoniae in the nasopharynx is thought to eventually lead to protective immunity. The role of anti-protein serum antibodies in mediating this immunity is unclear but vaccines incorporating pneumococcal proteins are being developed. We investigated the immunogenicity of individual proteins following pneumococcal colonisation in Kenyan and Burmese infants to establish whether antigen immunodominance is similar in diverse communities. Nasopharyngeal swabs to detect pneumococcal colonisation and serum samples at various time points were obtained in two separate studies from Burmese refugee and Kenyan infants. Sera was analysed for IgG to 27 pneumococcal proteins using multiplex electrochemiluminescence and titres compared between the cohorts. Prevalence of nasopharyngeal colonisation was compared at 18 and 36 weeks. Statistical analysis was performed on log-transformed data using R. Significance was defined of $p$-value less than 0.05 . Cross sectional prevalence of carriage at 18 weeks was $83 \%$ and $81 \%$ and at 36 weeks was $81 \%$ and $86 \%$ in the Kenyan and Burmese cohorts, respectively. When comparing IgG to individual proteins, the top 8 immuno-dominant proteins were identical between the two cohorts. Burmese infants had significantly higher birth IgG titres than the Kenyan cohort to $22 / 27$ antigens. However the ratio of $9 \mathrm{~m}$ :Birth IgG titres were significantly higher for the Kenyan cohort whose titres at 9 months were higher to all antigens (15/27 significantly). Despite differences in the kinetics of IgG production after birth, similar antigens were immunodominant in these two diverse populations. This data suggests that vaccines based on protein antigens are likely to have universal value.

No conflict of interest 
ISPPD-0162

Pneumococcal Colonization and Carriage

\title{
SERUM IGG AND SALIVARY IGA RESPONSES TO PNEUMOCOCCAL PROTEINS: DATA FROM A LONGITUDINAL CARRIAGE STUDY
}

\author{
N. Green ${ }^{1}$, L. Grant ${ }^{2}$, R. Reid'², R. Weatherholtz ${ }^{2}$, M. Santosham², K.L. O'Brien², D. Goldblatt ${ }^{1}$ \\ ${ }^{1}$ Immunobiology, UCL Institute of Child Health, London, United Kingdom; ${ }^{2}$ Bloomberg School of Public Health, John Hopkins University, Balti- \\ more, USA
}

The immune response to colonisation is important in the pathogenesis of Streptococcus pneumoniae but the role of anti-protein antibodies is unclear. To assess this we studied the relationship between anti-protein serum lgG, salivary IgA (sal-IGA) and pneumococcal colonisation among American Indian children and adults. Adults and children (mean age $4 \mathrm{y} 3 \mathrm{~m}$ ) had nasopharyngeal swabs taken monthly for 6 months to detect colonisation and saliva and serum collected at months 0 and 6 . Individuals who became colonized at any time were age matched to those never colonized. IgG and sal-IgA titres to 27 pneumococcal proteins were measured using multiplexed electrochemiluminescence. Overall for the entire cohort, adults had higher IgG and sal-IgA than children for $24 / 27$ proteins. At entry to the study, only IgG to pneumolysin correlated with protection against pneumococcal acquisition, and this only in adults. In adults who became colonised an increase in sal-IgA to 15/27 proteins with no significant changes in IgG was seen. In contrast children acquiring pneumococcus had higher IgG titres to $15 / 27$ proteins at month 6 compared with month 0 , but no changes in sal-lgA. There were no changes in antibody titres during the study in uncolonised adults or children. Pneumococcal colonisation stimulated different patterns of immune responses in adults and children. Previous antigen experience and the maturity of the immune system appears critical in determining the serological outcome of pneumococcal acquisition in the nasopharynx. Of the proteins studied, only anti-pneumolysin serum responses correlated with protection against pneumococcal acquisition, and this was only observed in adults.

No conflict of interest

ISPPD-0504

Pneumococcal Colonization and Carriage

\section{SIGNIFICANT IMPACT OF CONJUGATE 13-VALENT VACCINE ON STREPTOCOCCUS PNEUMONIAE CARRIAGE IN CHILDREN IN POLAND}

\author{
P. Grzesiowski ${ }^{1}$, M. Patrzalek ${ }^{2}$, D. Pawlik ${ }^{3}$ \\ ${ }^{1}$ Research and Innovation Dept., Prevention Infection Institute, Warsaw, Poland; ${ }^{2}$ Pediatric, Childrens Hospital, Kielce, Poland; ${ }^{3}$ Microbiology, \\ Primary Hospital, Makow Maz., Poland
}

Introduction: Streptococcus pneumoniae (SP) vaccination induces general and local immune response. According to our preliminary results, in vaccinated children, reduction in penicillin resistance in nasopharyngeal isolates have been found. As the further step of the study, for confirmation of phenotype difference, the serotype distribution has been investigated.

Aim: The study was conducted to assess the serotype distribution in nasopharyngeal carriage in pediatric population vaccinated with a conjugate 13-valent vaccine (PCV13) as compared to non-vaccinated children. Methods: All SP isolates obtained during the randomized, prospective study has been performed to assess the serotype distribution of SP strains isolated from carriage among 750 children aged 17 months-60 months of age. The study group consisted of 359 fully, age appropriately immunized children by using PCV13 within the official vaccination program in the city of Kielce (schedule - at 3, 5, 14 months of life) and 391 children not vaccinated against pneumococci living in the same region (the city of Ostrowiec).

Nasopharyngeal swabs were obtained by trained medical personnel, cultured in certified laboratory, SP isolates have been tested by using the Quellung method in reference laboratory according to standard previously described procedure.

Results: The prevalence of pneumococcal carriage was similar in vaccinated and non-vaccinated children $(28,4 \%$ vs $27,6 \%$ respectively). The serotype analysis in vaccinated carriers showed only $5 \%$ serotypes covered by the PCV 13 in comparison to $57 \%$ in non-vaccinated population $(p<0.0001)$.

Conclusion: Vaccination with PCV13 has significant impact on the reduction of vaccine covered serotypes in SP carriers and correlates with penicillin resistance of these isolates.

No conflict of interest 
ISPPD-0381

Pneumococcal Colonization and Carriage

\section{PNEUMOCOCCAL CARRIAGE - YEARLY SEROTYPE FLUCTUATIONS IN CHILDREN ATTENDING DAY CARE CENTRES}

H. Erlendsdóttir ${ }^{1}$, K. Halldórsson², K. Hauksson², B.D. Ingu-Andrésardóttir², A.J. Jónsson², A. Sæmundsson², K.G. Kristinsson ${ }^{3}$, Á. Haraldsson ${ }^{4}$

${ }^{1}$ Clinical Microbiology, Landspitali University Hospital \& University of Iceland, Reykjavík, Iceland; ${ }^{2}$ Faculty of Medicine, University of Iceland, Reykjavík, Iceland; ${ }^{3}$ Clinical Microbiology, Landspitali University Hospital and University of Iceland, Reykjavík, Iceland; ${ }^{4}$ Children's Hospital Iceland, Landspitali The National University Hospital of Iceland \& University of Iceland, Reykjavík, Iceland

Introduction: Pneumococci commonly colonize the nasopharynx of children. Iceland introduced pneumococcal conjugate vaccine (PCV-10) in the childhood vaccination program in 2011. The aim was to investigate changes in serotype prevalence and antimicrobial susceptibility before vaccinated children attend the day care centres. Material and Methods: Nasopharyngeal samples were collected annually from healthy children age 2-6 years, attending fifteen day care centres in the greater Reykjavík area during 2009-2013. The children's parents answered a questionnaire about antibiotic use during the 6 months before the sampling.

Results: The number of children sampled annually varied between 420 and 516 . The mean carriage rate of pneumococci was between $56 \%$ and $72 \%$, decreasing significantly with age (for 2013 , each additional year of age: odds ratio $(O R)=0.81, p=0.016)$. The proportion of children carrying penicillin non-susceptible pneumococci (PNSP) was 5-8\%, also decreasing with age (for 2013, each additional year of age: OR=0.61, $p=0.026$ ). The PNSP was highest among children receiving antibiotics up to 30 days prior to sampling (for 2013: $\mathrm{OR}=2.6, p=0.026$ ). The serotype proportions varied considerably from year to year for most of the serotypes. The most common types were 6B (13\%) in 2009, 23F (15\%) 2010, NT (11\%) 2011, 6A (24\%) 2012 and 19F (10\%) 2013.

Conclusion: Considerable fluctuations were noted for most of the serotypes. Only a minority of the individuals studied had received the PCV-vaccination. The natural fluctuations in serotype prevalence need to be considered in studies of vaccine efficacy.

Conflict of interest

ISPPD-0050

Pneumococcal Colonization and Carriage

\section{NASOPHARYNGEAL CARRIAGE AND LOWER AIRWAY INFECTION IN AUSTRALIAN INDIGENOUS CHILDREN WITH BRONCHIECTASIS: PNEUMOCOCCAL SEROTYPES AND AZITHROMYCIN RESISTANCE BY VACCINE GROUP}

K.M. Hare ${ }^{1}$, H. Smith-Vaughan ${ }^{1}$, A.J. Leach ${ }^{1}$, A.C. Cheng ${ }^{2}$, A.B. Chang ${ }^{1}$

${ }^{1}$ Child Health Division, Menzies School of Health Research, Darwin, Australia; ${ }^{2}$ Department of Epidemiology and Preventive Medicine, Monash University, Melbourne, Australia

Background and Aims: Indigenous children in the Northern Territory have very high rates of bronchiectasis. PCV7 was introduced in 2001 and replaced by PHiDCV in October 2009 and PCV13 in October 2011. We investigated the impact of vaccination on nasopharyngeal (NP) carriage and lower airway infection (LAI) in these children, many of whom receive long-term azithromycin therapy.

Methods: Children were enrolled, and NP swabs and bronchoalveolar lavage (BAL) fluid were collected under anaesthetic, stored at $-80^{\circ} \mathrm{C}$ and processed, as previously described. LAI was defined as $>10^{4} \mathrm{CFU} / \mathrm{mL}$ BAL fluid. Data were recorded on vaccines administered since birth. Azithromycin resistance was defined as MIC $>0.5 \mathrm{mg} / \mathrm{L}$. Results: From 2007 to 2013, 155 Indigenous children aged 5.2 to 154.6 (median 27.1) months with HRCT-confirmed bronchiectasis were enrolled; 89 (57\%) were male. There were no significant differences in NP carriage or LAl between vaccine groups (table).

Table 1: Streptococcus pneumoniae (Spn) NP carriage and lower airway infection

\begin{tabular}{|c|c|c|c|}
\hline $\begin{array}{l}\text { Vaccine group } \\
\text { ( } \geq 2 \text { doses) }\end{array}$ & $\begin{array}{l}\text { PCV7 } \\
(n=98)\end{array}$ & $\begin{array}{l}\text { PHiDCV } \\
(n=41)\end{array}$ & $\begin{array}{l}\text { PCV13 } \\
(n=16)\end{array}$ \\
\hline Nasopharyngeal carriage & $\begin{array}{l}\text { Spn }=41 \% \\
\text { AziRt=15\% }\end{array}$ & $\begin{array}{l}\text { Spn }=37 \% \\
\text { AziRt=29\% }\end{array}$ & $\begin{array}{l}\text { Spn }=31 \% \\
\text { AziRt=19\% }\end{array}$ \\
\hline Serotypes* & $\begin{array}{l}6 \mathrm{C} \times 4,16 \mathrm{~F} \times 4,23 \mathrm{~F} \times 4, \\
15 \mathrm{~B} \times 3,22 \mathrm{~F} \times 3,9 \mathrm{~N} \times 2, \\
11 \mathrm{~A} \times 2,19 \mathrm{~A} \times 2,21 \times 2 \\
23 \mathrm{~B} \times 2,24 \times 2,6 \mathrm{~A}, 6 \mathrm{~B}, \\
7 \mathrm{C}, 10 \mathrm{~A}, 14,15 \mathrm{~A}, 15 \mathrm{C} \\
17 \mathrm{~F}, 19 \mathrm{~F}, 22 \mathrm{~A}, 23 \mathrm{~A}, 35 \mathrm{~B}\end{array}$ & $\begin{array}{l}15 \mathrm{~A} \times 4,15 \mathrm{~B} \times 4,15 \mathrm{C} \times 3 \\
17 \mathrm{~F} \times 2,6 \mathrm{C}, 7 \mathrm{~B}, 9 \mathrm{~V}, 13,19 \mathrm{~A}\end{array}$ & $\begin{array}{l}13,15 B, 15 C \\
19 A, 23 A, 23 B\end{array}$ \\
\hline Lower airway infection & $\begin{array}{l}\text { Spn }=16 \% \\
\text { AziRt }^{+}=10 \%\end{array}$ & $\begin{array}{l}\text { Spn }=24 \% \\
\text { AziRt }=20 \%\end{array}$ & $\begin{array}{l}\text { Spn }=13 \% \\
\text { AziRt }=0 \%\end{array}$ \\
\hline Serotypes* & 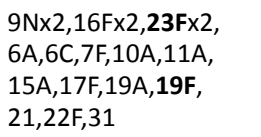 & $\begin{array}{l}15 \mathrm{~A} \times 3,6 \mathrm{C}, 7 \mathrm{~B}, 13,15 \mathrm{~B} \\
17 \mathrm{~F}, 19 \mathrm{~A}, 19 \mathrm{~F}, 22 \mathrm{~F}\end{array}$ & $23 \mathrm{~A}, 23 \mathrm{~B}$ \\
\hline
\end{tabular}


†AziR, azithromycin-resistant Spn strain(s) as \% children (80\% of resistant strains had MIC $\geq 16 \mathrm{mg} / \mathrm{L}$ ); *Vaccine serotypes in bold, AziR serotypes in italics.

Conclusion: There are currently insufficient data to draw conclusions regarding vaccine impact. Temporal trends and the emergence of multi-resistant non-vaccine serotypes (e.g. 15A) also need to be considered. PCV studies are ongoing.

No conflict of interest

ISPPD-0300

Pneumococcal Colonization and Carriage

\title{
EFFECT OF ANTIRETROVIRAL THERAPY ON PNEUMOCOCCAL CARRIAGE IN HIV-POSITIVE ADULTS IN KARONGA DISTRICT, MALAWI
}

\author{
E. Heinsbroek ${ }^{1}$, J.M. Read ${ }^{2}$, T. Tafatatha ${ }^{3}$, C. Mwafulirwa ${ }^{3}$, A. Phiri ${ }^{3}$, N. French ${ }^{1}$
}

${ }^{1}$ Department of Clinical Infection Microbiology \& Immunology, Institute of Infection and Global Health, Liverpool, United Kingdom; ${ }^{2}$ Department of Epidemiology and Population Health, Institute of Infection and Global Health, Liverpool, United Kingdom; ${ }^{3}$ Karonga Prevention Study, London School of Hygiene and Tropical Medicine, Chilumba, Malawi

Background: HIV-positive adults in Malawi have high rates of pneumococcal carriage and invasive disease and are key targets for herd protection. We investigated the effect of antiretroviral therapy (ART) on pneumococcal carriage in a cohort of HIV-positive adults prior to infant PCV roll-out.

Methods: We recruited HIV-positive adults newly attending a rural HIV clinic in northern Malawi between February 2008 and May 2010. Nasopharyngeal samples were taken at baseline and after 6, 12, 18, 24 months. We included all individuals with a baseline and at least one follow-up result. We compared pneumococcal carriage by ARTstatus using generalized linear mixed models adjusted for baseline CD4count, CD4count at sampling time, gender, seasonality and number of children $<5$ years in the household.

Results: In total, 335 individuals were included, of which 223 individuals started ART during the follow-up period. Pneumococcal carriage prevalence at baseline did not differ by individuals receiving ART during follow-up or not ( $21.0 \%$ vs. $20.6 \%, p=0.99)$. Individuals who received ART had higher pneumococcal carriage than individuals who did not receive ART (25.5\% vs. $19.6 \%, p=0.03)$, particularly for serotypes not included in PCV13 $(15.9 \%$ vs. $9.5 \% p=$ 0.004). Following adjustment, increased odds of carriage were still observed for individuals on ART, but results were non-significant (all serotypes: OR1.36, 95\% Cl 0.85-2.17, $p=0.20$; non-PCV13 serotypes: OR1.90, 95\%Cl 0.98-3.68, $p$ $=0.06)$.

Conclusion: Pneumococcal carriage in HIV-positive adults in Malawi remained high despite start of ART, with a tendency to increased carriage of non-PCV13 serotypes. HIV-positive adults on ART remain an important reservoir for pneumococcal diversity.

No conflict of interest

\section{ISPPD-0306 \\ Pneumococcal Colonization and Carriage}

\section{DECREASE IN PNEUMOCOCCAL CARRIAGE BEFORE INTRODUCTION OF THE PNEUMOCOCCAL CONJUGATE VACCINE IN KARONGA DISTRICT, MALAWI}

\author{
E. Heinsbroek ${ }^{1}$, J.M. Read ${ }^{2}$, T. Tafatatha ${ }^{3}$, A. Phiri' ${ }^{3}$, C. Chisambo ${ }^{3}$, B. Ngwira ${ }^{4}$, N. French ${ }^{1}$ \\ ${ }^{1}$ Department of Clinical Infection Microbiology \& Immunology, Institute of Infection and Global Health, Liverpool, United Kingdom; ${ }^{2}$ Depart- \\ ment of Epidemiology and Population Health, Institute of Infection and Global Health, Liverpool, United Kingdom; ${ }^{3}$ Karonga Prevention Study, \\ London School of Hygiene and Tropical Medicine, Chilumba, Malawi; ${ }^{4}$ Malawi-Liverpool-Wellcome Clinical Research Programme, College of Med-
} icine, Blantyre, Malawi

Background: Decreasing trends in invasive pneumococcal disease have been observed in Malawi in recent years. We investigated whether this decrease was reflected in pneumococcal carriage dynamics, by studying seasonal and long-term patterns in pneumococcal carriage in Karonga, northern Malawi, over a three-year period.

Methods: Mother/infant pairs were recruited in a longitudinal study investigating the effect of maternal HIV-status on infant pneumococcal acquisition. Nasopharyngeal swabs were collected monthly for one year from infants, mothers and siblings $<5$ years of age. Samples were collected between January 2009 and November 2011, before introduction of pneumococcal conjugate vaccine. We used generalized additive mixed models to examine seasonal and long-term trends in pneumococcal carriage incidence, adjusted for age and maternal HIV-status.

Results: In total, 185 mother/infant pairs and 140 siblings were included in the study. Pneumococcal incidence was seasonal, with the highest incidence in the cold season $(p<0.001)$. A decreasing trend in pneumococcal incidence was observed in all age groups over the three-year study period $(p<0.001)$. For serotype 19A an upward trend was observed for infants $(p<0.001)$ and siblings $(p=0.01)$. In infants, pneumococcal incidence decreased from $49.3 \%$ $(95 \% \mathrm{Cl} 42.4-56.2 \%)$ in the cold season in 2009 to $38.4 \%(95 \% \mathrm{Cl} 32.6-44.5 \%)$ and $22.1 \%(95 \% \mathrm{Cl} 14.2-31.8 \%)$ in the same months in 2010 and 2011.

Conclusion: Before introduction of pneumococcal vaccination in Malawi, pneumococcal carriage incidence dramatically decreased during the study period. Seasonal and long-term trends need to be taken into account when evaluating vaccine effectiveness, in particular when a before-after design is used.

No conflict of interest 


\title{
PNEUMOCOCCAL CARRIAGE RATES REMAIN HIGH IN WESTERN AUSTRALIAN ABORIGINAL PEOPLE: 12 YEARS AND TWO VACCINES LATER
}

\author{
A. Hoskins ${ }^{1}$, D. Collins ${ }^{1}$, K. Senasinghe ${ }^{1}$, K. Hare ${ }^{2}$, J. Bowman ${ }^{3}$, J. Jones ${ }^{3}$, N.A. Stemberger ${ }^{3}$, T.V. Riley ${ }^{3}$, H. Smith- \\ Vaughan ${ }^{2}$, A.J. Leach ${ }^{2}$, D. Lehmann ${ }^{1}$ \\ ${ }^{1}$ Population Sciences, Telethon Institute for Child Health Research University of Western Australia, Perth, Australia; ${ }^{2}$ Child Health Division, \\ Menzies School of Health Research, Darwin, Australia; ${ }^{3}$ Divisions of Microbiology \& Infectious Diseases, PathWest Laboratory Medicine, Perth, \\ Australia
}

Background: Monitoring nasopharyngeal carriage complements invasive pneumococcal disease surveillance and provides information on circulating serotypes. We report the impact of 13-valent pneumococcal conjugate vaccine (13vPCV) on nasopharyngeal carriage in Western Australian Aboriginal people after 13vPCV replaced 7vPCV in the national immunization program in July 2011.

Methods: Nasopharyngeal swabs (NPS) were collected opportunistically from Aboriginal children and adults living in urban, rural and remote areas of Western Australia three years before and two years after 13vPCV was given to Aboriginal children in a 2-4-6-month schedule. Specimens were cultured using selective media and pneumococci serotyped using the Quellung reaction.

Results: Among 823 NPS cultured post-introduction of $13 \mathrm{vPCV}$, the pneumococcal carriage rate in children $<5 y$ rs was $65 \%$ and $31 \%$ in people $\geq 5$ yrs compared with $72 \%$ and $35 \%$, respectively, in 1500 NS collected pre-introduction of 13vPCV. The most common serotypes following introduction of $13 \mathrm{vPCV}$ were $16 \mathrm{~F}(9 \%), 19 \mathrm{~F}(9 \%), 11 \mathrm{~A}(7.5 \%)$ $6 \mathrm{C}(7.5 \%)$, and 19A (7\%) in children <5yrs and 19F (9\%), 10A (6\%), 15B (6\%), 6A (5\%) and 6C (5\%) in older people. Carriage of the six additional 13vPCV serotypes declined from $18.3 \%$ to $15.0 \%$ in children $<5 y$ rs.

Conclusion: Overall pneumococcal carriage rates in West Australian Aboriginal people remain high with limited decline post-13vPCV introduction. Three of the most commonly carried serotypes in children <5yrs are not covered by PCV13 while serotype 19F (included in PCVs since 2001) continues to be commonly carried. Ongoing surveillance is needed to follow the longer term impact of $13 \mathrm{vPCV}$, identify emerging serotypes and inform future vaccine development.

No conflict of interest

ISPPD-0266

Pneumococcal Colonization and Carriage

\section{PNEUMOCOCCAL NASOPHARYNGEAL CARRIAGE IN SLOVAK CHILDREN UP TO 5 YEARS OF AGE}

\author{
H. Hupkova ${ }^{1}$, M. Macaj ${ }^{2}$
}

${ }^{1}$ Institute of Microbiology, Comenius University School of Medicine, Bratislava, Slovakia; ${ }^{2}$ Pediatric ORL Department of Children Faculty Hospital in Bratislava, Comenius University School of Medicine, Bratislava, Slovakia

Background and Aims: Bacterial spectrum of upper respiratory tract in children is mostly reflecting in nasopharyngeal carriage. Nasopharyngeal colonization of Streptococcus pneumoniae is asymptomatic in $50 \%$, though in case of invasive and non-invasive pneumococcal infections nasopharyngeal carriage has significant role in etiology. Influence of pneumococcal vaccination reduced nasopharyngeal carriage of S. pneumoniae however replacement phenomenon has been observed.

Methods: Nasopharyngeal swabs $(n=206)$ and adenoid tissue $(n=206)$ in 206 healthy infants children before adenoidectomy was examined and the bacterial nasopharyngeal carriage was identified in period from March 2012 up to July 2013. In case of S. pneumoniae serotyping was performed by Quellung method.

Results: Nasopharyngeal carriage of S. Pneumoniae 43, (13\%), Staphylococcus aureus 40, (63\%), Haemophilus influenzae $30 \%$, Moraxella catarrhalis $15 \%$ and Streptococcus pyogenes 4, (38\%) were identified respectively. Serotyping manifested dominant role of serotype $19 \mathrm{~A}(21,33 \%)$ although replacement phenomenon of non vaccine serotypes (36\%) increased dramatically. Overall, 12\% (PCV 7), 14,67\% (PCV 10), 48\% (PCV 13) of the isolates belonged to strains covered by the pneumococcal conjugate vaccine. [01] Most pneumococci were resistant to one or more antimicrobial classes. The rate of penicillin resistance MIC > 1.0 was relatively low (15.\%); macrolide (Erytromycine) resistance was high (48,45\%).[02]

Conclusion: Relation between nasopharyngeal carriage and pneumococcal invasive and non-invasive infections as otitis media is obvious although nasopharyngeal carriage of $S$. pneumoniae and pneumococcal replacement phenomenon in nasopharyngeal carriage has impact on infections caused by new pneumococcal serotypes.

No conflict of interest 
ISPPD-0068

Pneumococcal Colonization and Carriage

\title{
THE IMMUNOMODULATORY EFFECT OF MACROLIDES PROMOTE THE CLEARANCE PNEUMOCOCCAL NASOPHARYNGEAL COLONIZATION IN MICE
}

N. Iwanaga ${ }^{1}$, S. Nakamura ${ }^{1}$, K. Oshima ${ }^{1}$, T. Kajihara ${ }^{1}$, T. Takazono ${ }^{1}$, Y. Imamura ${ }^{1}$, K. Yanagihara ${ }^{2}$, K. Izumikawa ${ }^{1}$, T. Sunazuka ${ }^{3}$, S. Omura ${ }^{3}$, S. Kohno ${ }^{1}$

${ }^{1}$ Department of Molecular Microbiology and Immunology, Nagasaki University Graduate School of Biomedical Sciences, Nagasaki, Japan; ${ }^{2}$ Department of Laboratory Medicine, Nagasaki University Hospital, Nagasaki, Japan; ${ }^{3}$ Kitasato Institute for Life Sciences, Kitasato University, Tokyo, Japan

Background and Aims: Streptococcus pneumoniae (the pneumococcus) colonizes the mucosal surfaces of the human upper respiratory tract (URT), and occasionally leads to invasive disease. Regulation of nasopharyngeal colonization could be a candidate for preventing pneumococcal infections. We investigated whether macrolides, which have variant immunomodulatory effects, could promote the clearance of pneumococcal colonization via immunomodulation.

Methods: Peritoneal macrophages extracted from mice were pretreated with clarithromycin (CAM) and azithromycin (AZM). The levels of cytokines in the supernatant were determined by ELISA and cytokine array, and intracellular chemokine analysis was performed by flow cytometry. The cell signaling pathway was also analyzed by westernblotting and gene silencing assay. The macrolides-mediated pneumococcal clearance was examined by using intranasal bacterial challenge in mice. EM900 (a novel 12-membered non-antibiotic macrolides but has an immunomodulatory effect) was orally administered throughout this experiment. URT lavages were analyzed for the kinetics of colonization, the cellular response and inflammatory cytokines.

Results: The production and mRNA expression of CCL2 of peritoneal macrophages were significantly induced by CAM and AZM treatment in a dose-dependent manner. Intracellular CCL2 was also detected by flow cytometry. Westernblotting showed phosphorylation of NF-kB 15 minutes after macrolides-treatment. The density of pneumococcus in URT was significantly decreased in EM900 treated mice compared to untreated mice at 14 days after pneumococcal inoculation. Coincidentally, the recruitment of macrophages and CCL2 mRNA expression in nasal cavity were significantly increased by administration of EM900.

Conclusion: Immunomodulatory action of macrolides promotes the clearance of pneumococcal nasopharyngeal colonization by inducing the accumulation of macrophages to URT.

No conflict of interest

ISPPD-0281

Pneumococcal Colonization and Carriage

\section{EVALUATION OF STREPTOCOCCUS SALIVARIUS AS A PROBIOTIC INHIBITOR OF PNEUMOCOCCAL COLONISATION IN VITRO AND IN MICE}

\author{
M. John ${ }^{1}$, E.M. Dunne ${ }^{2}$, P.V. Licciardi ${ }^{3}$, C. Satzke ${ }^{4}$, O. Wijburg ${ }^{5}$, R.M. Robins-Browne ${ }^{6}$, S. O' Leary $^{7}$ \\ ${ }^{1}$ Otolaryngology, Christian Medical College, Vellore, India; ${ }^{2}$ Pneumococcal Research, Murdoch Childrens Research Institute, Royal Children's Hos- \\ pital, Melbourne, Australia; ${ }^{3}$ Pneumococcal Research, Allergy and Immune Disorders, Murdoch Childrens Research Institute, Royal Chil- \\ dren's Hospital, Melbourne, Australia; ${ }^{4}$ Pneumococcal Research, Infectious Diseases \& Microbiology Department of Microbiology \& Immunology, \\ Murdoch Childrens Research Institute, Royal Children's Hospital The University of Melbourne, Melbourne, Australia; ${ }^{5}$ Department of Microbiolo- \\ gy \& Immunology, The University of Melbourne, Melbourne, Australia; ${ }^{6}$ Department of Microbiology \& Immunologylnfectious Diseases \& Micro- \\ biology, The University of Melbourne Murdoch Childrens Research Institute Royal Children's Hospital, Melbourne, Australia; ${ }^{7}$ Department of Oto- \\ laryngology, The University of Melbourne, Melbourne, Australia
}

Background and Aims: Nasopharyngeal colonisation is the initial step in pathogenesis of pneumococcal diseases, including otitis media. Pneumococcal vaccines are less effective against otitis media than they are against systemic pneumococcal infections. Previous studies have suggested that Streptococcus salivarius, a commercial probiotic originally isolated from the throat, could be a potential pharyngeal probiotic. We investigated the effects of $S$. salivarius on pneumococcal colonisation and otitis media by using in vitro and in vivo models of infection. Methods: High (5 to 10 times more than pneumococci), medium (approximately equal) or low (5 to 10 times less) numbers of $S$. salivarius were added before, with, or after pneumococci (pre-, co-, and post-administration respectively) to human epithelial CCL-23 cells in vitro. The percent colonisation of pneumococci was determined after $3 \mathrm{~h}$ incubation. In experiments using infant mice, repeated pernasal administration of S. salivarius ( $\sim 10^{7}$ colony forming units (CFU)/dose) was used as an intervention in a model of pneumococcal colonisation and otitis media. bThere was a time-dependent (pre-administration more effective than co- and post-administration) and dosedependent (high numbers more effective than medium or low numbers) inhibition of pneumococcal adherence to CCL-23 cells by S. salivarius. However, S. salivarius colonised the nasopharynx of mice poorly, and did not alter pneumococcal colonisation levels or the development of otitis media in vivo.

Conclusion: Our data indicated that $S$. salivarius can inhibit pneumococcal colonisation in vitro, but not in infant mice. Further well-designed human trials are warranted to evaluate $S$. salivarius as a potential probiotic to reduce otitis media.

No conflict of interest 


\title{
HIGH MULTIPLE-CARRIAGE OF DIVERSE PNEUMOCOCCAL SEROTYPES AND GENETIC VARIATION AT THE CAPSULAR POLYSACCHARIDE LOCUS OF VACCINE SEROTYPES IN MALAWIAN CHILDREN
}

\author{
A.W. Kamng'ona ${ }^{1}$, J. Hinds ${ }^{2}$, N. Nar-Zeev ${ }^{3}$, K. Gould ${ }^{2}$, C. Chaguza ${ }^{4}$, C. Msefula ${ }^{5}$, J. Cornick ${ }^{6}$, B. Kulohoma ${ }^{6}$, K. Gray $^{6}$,

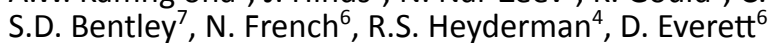 \\ ${ }^{1}$ The Microbe Immunity and vaccines, Malawi-Liverpool-Wellcome Trust Clinical Research Programme, Blantyre, Malawi; ${ }^{2}$ Bacterial Microar- \\ ray Group at St George's ( BuG@S), St George's University of London, London, United Kingdom; ${ }^{3}$ Karonga Prevention Study, Karonga Preven- \\ tion Study, Karonga, Malawi; ${ }^{4}$ The Microbe Immunity and vaccines, Malawi-Liverpool Wellcome Trust, Blantyre, Malawi; ${ }^{5}$ Microbiology, Universi- \\ ty of Malawi College of Medicine, Blantyre, Malawi; ${ }^{6}$ Institute of Infection and Global Health, University of Liverpool, Liverpool, United Kingdom; \\ ${ }^{7}$ Pathogen Genomics, Wellcome Trust Sanger Institute, Cambridge, United Kingdom
}

Background and Aims: Pneumococcal carriage is a pre-requisite for invasive pneumococcal disease, yet there are limited data on multiple serotype carriage in sub-Saharan Africa. Multiple carriage promotes genetic recombination, which may lead to vaccine escape. The aim of this study was to determine the prevalence of multiple carriage in Malawian children in the context of HIV infection.

Methods: Nasopharyngeal samples were collected from HIV-infected $(n=44)$ and uninfected $(n=72)$ Malawian children. Pneumococcal strains were characterised by microarray serotyping and whole genome sequencing. Results: 43 distinct and 5 non-typeable serotypes were identified. Multiple carriage was detected in 40\% (46/116) of the children, and was unaffected by HIV status. However, carriage of serotype 3 was significantly higher in HIV positive $(6 / 44)$ than HIV negative $(1 / 72)$ subjects $(p=0.028 ; \mathrm{OR}=11.2)$ Capsular polysaccharide locus variants were observed and occured naturally in serotypes 6B, 19A, and 20. The 6B variants harboured an intact allele of HG263 gene, while a frame-shift mutation was observed in the wild type. Unlike the wild type, the 19A-and 20-variants contained an inversion in the sugar-biosynthesis $(\mathrm{rm} / \mathrm{D})$ gene and a deletion of the transferase (whaF) gene, respectively.

Conclusion: There is a high degree of serotype diversity and multiple carriage in Malawian children, which could drive genetic recombination and vaccine escape. Further studies are recommended in this setting, to establish if PCV-13 would offer cross-protection againts these variants

No conflict of interest

ISPPD-0071

Pneumococcal Colonization and Carriage

\section{PNEUMOCOCCAL CARRIAGE IN CHILDREN, THEIR PARENTS AND OLDER ADULTS FOLLOWING THE INTRODUCTION OF THE SEVEN-VALENT PNEUMOCOCCAL CONJUGATE VACCINE}

M. Hamaluba ${ }^{1}$, R. Kandasamy ${ }^{1}$, S. Ndimah ${ }^{1}$, R. Morton $^{2}$, M. Caccamo $^{1}$, H. Robinson ${ }^{1}$, S. Kelly ${ }^{1}$, A. Field ${ }^{1}$, L. Norman $^{1}$, E. Plested ${ }^{1}$, B.A.V. Thompson ${ }^{3}$, S. Kerridge ${ }^{1}$, R. Lazarus ${ }^{1}$, T. John ${ }^{1}$, J. Holmes ${ }^{4}$, P. Waight ${ }^{5}$, J. Hinds ${ }^{6}$, D. Crook ${ }^{7}$, M.D. Snape ${ }^{1}$, A.J. Pollard ${ }^{1}$

${ }^{1}$ Paediatrics, University of Oxford, Oxford, United Kingdom; ${ }^{2}$ Paediatrics, Guy's and St Thomas' National Health Service Foundation Trust, London, United Kingdom; ${ }^{3}$ Primary Healthcare Sciences, University of Oxford, Oxford, United Kingdom; ${ }^{4}$ Centre for Statistics in Medicine, University of Oxford, Oxford, United Kingdom; ${ }^{5}$ Health Protection Services, Public Health England, London, United Kingdom; ${ }^{6}$ Cellular and Molecular Medicine, University of London, London, United Kingdom; ${ }^{7}$ Microbiology, University of Oxford, Oxford, United Kingdom

Background: Using nasopharyngeal carriage as a marker of vaccine impact, pneumococcal colonisation and its relation to invasive disease and demographic attributes were examined in children, their parents, and older adults in the UK following the introduction of PCV7 and prior to PCV13.

Methods: Nasopharyngeal swabs were collected, between November 2010 and September 2011, from children 25-55 months of age who had previously received three doses of PCV7, their parents, and adults aged 65 years and over. Pneumococcal serotyping was performed according to WHO guidelines with non-typeable isolates further analysed by comparative genomic hybridisation (CGH) microarray. Surveillance of invasive pneumococcal disease (IPD) in England and Wales was conducted throughout the corresponding time period.

Results: Pneumococcus was isolated from $47 \%$ of children, $9 \%$ of parents and $2.2 \%$ of older adults. For the corresponding groups the proportion of serotypes covered by PCV7 were $1.5 \%, 0 \%$ and $15.4 \%$, with a further $20 \%$, $44.4 \%$ and $7.7 \%$ coverage added by those in PCV13. In each group the proportion of IPD due to the serotypes covered by PCV7 were $1.0 \%, 7.4 \%$ and $5.1 \%$ with a further $65.3 \%, 42.1 \%$ and $61.4 \%$ attributed to those in PCV 13 . A significant correlation between contact with children and pneumococcal carriage in older adults was noted.

Conclusion: In this population the rate of carriage is highest in children and those exposed to them. Direct vaccine impact is exemplified by the low carriage and IPD rates of PCV7 serotypes in vaccinated children, whilst the indirect effects of herd protection are implied by similar observations in unvaccinated parents and older adults.

Conflict of interest 
ISPPD-0255

Pneumococcal Colonization and Carriage

\title{
PNEUMOCOCCAL CARRIAGE IN KENYAN CHILDREN BEFORE AND AFTER 10-VALENT PNEUMOCOCCAL CONJUGATE VACCINE (PCV10) INTRODUCTION, 2009 AND 2012
}

L. Kim ${ }^{1}$, L. Conklin ${ }^{1}$, G. Bigogo ${ }^{2}$, B. Fields ${ }^{3}$, A. Odoyo ${ }^{2}$, H. Odiembo ${ }^{2}$, M. Carvalho ${ }^{1}$, F. Pimenta ${ }^{1}$, K. Odero ${ }^{3}$, J. Milucky $^{1}$, D. Ouma ${ }^{2}$, A. Harris ${ }^{1}$, D. Feikin ${ }^{2}$, D. Burton ${ }^{3}$, J. Montgomery ${ }^{3}$, C. Van Beneden ${ }^{1}$, C. Whitney ${ }^{1}$, R. Breiman ${ }^{\prime}$

${ }^{1}$ Respiratory Diseases Branch, Centers for Disease Control and Prevention, Atlanta, USA; ${ }^{2}$ Global Disease Detection Division and International Emerging Infections Program, Kenya Medical Research Institute/Centers for Disease Control and Prevention Public Health and Research Collaboration, Kisumu, Kenya; ${ }^{3}$ Global Disease Detection Division and International Emerging Infections Program, Kenya Medical Research Institute/ Centers for Disease Control and Prevention Public Health and Research Collaboration, Nairobi, Kenya

Background and Aims: Kenya introduced 10-valent pneumococcal conjugate vaccine (PCV10) for infants at 6, 10, and 14 weeks in February 2011 without catch-up vaccination for older children. We evaluated PCV10 impact on pneumococcal carriage among children living in a crowded, informal settlement.

Methods: We conducted cross-sectional carriage surveys during October to December in 2009, before vaccine introduction, and 2012, after vaccine introduction, among randomly-selected children $<5$ years old in Kibera, an urban slum in Nairobi. Vaccination histories were verified. Nasopharyngeal swabs were placed in STGG media, and specimens were cultured for Streptococcus pneumoniae isolation after broth enrichment. Pneumococcal isolates were serotyped by multiplex-PCR and Quellung reaction.

Results: Among 545 children in 2009 and 309 in 2012, median ages were 2.3 and 1.0 years, respectively. The proportion of children $<5$ years colonized with pneumococci was similar in 2009 and $2012(90 \%$ and $92 \%$, respectively). Among all children, PCV10 serotypes were found in 38\% in 2009 compared to $17 \%$ in 2012 (percent change, $-54 \% ; p<0.001$ ). Serotypes with the greatest reductions were $19 \mathrm{~F}$ (percent change, $-67 \%$ ) and $9 \mathrm{~V}$ (percent change, $-65 \%)$. PCV10 serotypes decreased by $57 \%$ in infants $<12$ months $(p<0.001)$ and $59 \%$ in children $1-4$ years $(p<0.001)$. In a subset of unvaccinated children $1-4$ years old $(n=85)$, PCV10 serotypes decreased by $61 \%(p<$ 0.001).

Conclusion: Less than 2 years after PCV10 introduction, declines in PCV10-type colonization were substantial even in children not eligible for vaccination. Our data suggest that herd effects are seen early in a program without catchup vaccination.

No conflict of interest

ISPPD-0391

Pneumococcal Colonization and Carriage

\section{COMPARISON OF PNEUMOCOCCAL CARRIAGE IN CHILDREN WITH UPPER RESPIRATORY TRACT INFECTIONS AND HEALTHY CHILDREN}

\author{
P. Gudjónsson ${ }^{1}$, H. Erlendsdóttir², M.A. Hjálmarsdóttir², S.J. Quirk², A. Haraldsson ${ }^{3}$, K.G. Kristinsson² \\ ${ }^{1}$ Faculty of Medicine, University of Iceland, Reykjavík, Iceland; ${ }^{2}$ Clinical Microbiology, Landspitali University Hospital and University of Iceland, \\ Reykjavík, Iceland; ${ }^{3}$ Children's Hospital Iceland, Landspitali University Hospital and University of Iceland, Reykjavík, Iceland
}

Introduction: The nasopharynx of children is the main habitat of pneumococci. The difference in pneumococcal carriage between healthy children and children with upper respiratory tract infections (URTI) is usually speculative. The aim of the study was to compare serotypes and antibiotic susceptibilities of pneumococci in healthy carriage and children with URTI.

Material and Methods: A comparison was made between isolates collected during studies of pneumococcal carriage of children attending day care centres in 2009-2012 (DCC group), and nasopharyngeal clinical samples from children with upper respiratory tract infections (CS group) submitted to the microbiology laboratory at Landspitalinn during the same period. Comparisons were made between children of the same age.

Results: There were 1281 isolates from children in the DCC group and 770 in the CS group. The most common serotypes were $23 \mathrm{~F}, 6 \mathrm{~A}, 19 \mathrm{~F}, 6 \mathrm{~B}$ and $19 \mathrm{~F}, 6 \mathrm{~A}, 23 \mathrm{~F}, 6 \mathrm{~B}$ in the DCC and CS group respectively. The difference was significant for $19 \mathrm{~F}(p<0.0001), 23 \mathrm{~F}(p=0.02)$ and non-typable $(p<0.0001)$ isolates. Penicillin non-susceptible pneumococci were more common in the CS group (265 isolates, 34\%) compared to the DCC group (171 isolates, $14 \%)$, the difference being significant in the age groups 1.5-1.9 years $(p=0.03), 2-2.9$ years $(p=0.001)$ and $4-4.9$ years of age $(p=0.02)$. Multi-resistant Streptococcus pneumoniae was also more often found in the CS group (33\% vs. $14 \%)$.

Conclusion: There was a significant difference in carriage of the main serotypes between the two study groups and a significantly higher resistance rate in the CS group.

Conflict of interest 


\title{
STREPTOCOCCUS PNEUMONIAE ISOLATION FROM UPPER RESPIRATORY TRACT OF CHILDREN IN A RURAL COMMUNITY OF NORTH INDIA
}

\author{
S. Kumar ${ }^{1}$, B. Kumar Das ${ }^{1}$, A. Kapil ${ }^{1}$, R. Amarchand ${ }^{2}$, D. Ram Purakayastha ${ }^{2}$, V. Rastogi ${ }^{2}$, A. Wahi ${ }^{2}$, A. Krishnan ${ }^{2}$ \\ ${ }^{1}$ Department of Microbiology, All India Institute of Medical Science, Delhi, India; ${ }^{2}$ Center for Community Medicine, All India Institute of Medi- \\ cal Science, Delhi, India
}

Background and Aims: The carriage of Streptococcus pneumoniae in the nasopahrynx of children has been shown to precede disease and serves as a source of transmission in a community. We carried out the present study to determine the culture positivity and antibiotic susceptibility pattern of S. pneumoniae from nasopharynx of children.

Methods: As a part of community based Acute Respiratory Infection (ARI) surveillance in villages of Ballabhgarh Block of Haryana, North India. Naso-oropharyngeal swabs were collected from 1378 children suffering from ARI (ALRI-542, AURI-836) and 253 controls in the age group 0-10 years from Aug 2012 to Aug 2013. A total of 202 urine samples (ALRI-177 and 25 Controls) were also collected from these children to screen for pneumococcal antigen by BinaxNOW. Ethical approval was obtained from the Institutional review committee.

Results: A total of 68 children out of $1378(5 \%)$ having ARI, were culture positive for S.pneumoniae in

Nasopharyngeal swabs. Age wise distribution of S.pneumoniae culture positivity was 0-2 years -ALRI-28/316; AURI13/214, 2-5 years -ALRI-5/159, AURI-3/32 and 5-10 years -ALRI-3/67, AURI-7/302. Among controls, 9 were also positive in 0-2 yrs. All isolates were sensitive to penicillin \& resistant to co-trimoxazole. Erythromycin resistance was seen in 18\%. Of the 202 urine samples positivity for pneumococcal antigen was ALRI-88/177 and Control-12/25 with age wise distribution as 2-5yrs- ALRI-46/88, control-10/16.

Conclusion: The study showed that pneumococcal positivity among children was high in our community especially in the age groups of 0-2 years. All strains remain penicillin sensitive and cotrimoxazole resistant.

No conflict of interest

ISPPD-0447

Pneumococcal Colonization and Carriage

\section{THE IMPACT OF EARLY EXPOSURE TO A PNEUMOCOCCAL CONJUGATE VACCINE ON NASOPHARYNGEAL PNEUMOCOCCAL CARRIAGE AND MICROBIAL ECOLOGY}

B. Kwambana ${ }^{1}$, E. Foster-Nyarko ${ }^{1}$, F. Ceesay ${ }^{1}$, B. Herter ${ }^{2}$, O. Hall², M. Campise ${ }^{2}$, E. Sodergren², M. Barer ${ }^{3}$, R. Adegbola $^{4}, \mathrm{G}$. Weinstock ${ }^{2}$, M. Antonio ${ }^{1}$

${ }^{1}$ Vaccinology Theme, Medical Research Council Unit The Gambia, Fajara, Gambia; ${ }^{2}$ The Genome Center, Washington University in St. Louis, St. Louis, USA; ${ }^{3}$ Infection Immunity and Inflammation, University of Leicester, Leicester, United Kingdom; ${ }^{4} \mathrm{GSK}$, GSK, Brussels, Belgium

Background and Aims: Nasopharyngeal (NP) carriage of pneumococcus predisposes young children to invasive pneumococcal disease. This study characterizes the impact of the seven-valent pneumococcal conjugate vaccine (PCV7) on pneumococcal carriage and the microbiome in the first year of life.

Methods: NP swabs were collected from 102 subjects from birth to twelve months at regular intervals. G1 $(n=39)$ and $\mathrm{G} 2(n=30)$ subjects were from PCV7 naive communities and G3 $(n=39)$ subjects were from widely vaccinated communities. G1 subjects received PCV7 after 8 months while G2 and G3 subjects were vaccinated at 2, 3 and 4 months. Microbiology techniques were used to culture and serotype pneumococci. Microbiome analysis was conducted by bar-coded 16S-rRNA gene pyrosequencing.

Results: Overall pneumococcal carriage was $79 \%(95 \% \mathrm{Cl}: 75-82 \%), 75 \%(95 \% \mathrm{Cl}: 71-79 \%)$ and $82 \%(95 \% \mathrm{Cl}: 79-85 \%)$ for G1, G2 and G3 participants respectively. The prevalence of PCV7 serotypes was $29 \%$ (95\%Cl:24-34\%) among the G1 subjects, significantly higher than among subjects from G2 14\% (95\% Cl:11-18\%) and G3 5\% (95\%Cl:3-7\%) $p<$ 0.001. The most common PCV7 serotypes were 19F (4\%), $14(4 \%)$ and 23F (3\%) while the predominant non-vaccine serotypes were 19A (13\%), $13(4 \%), 35 \mathrm{~B}(4 \%)$ and 15B (3\%) among all the children. In the first eight months of life, there were 6-fold more significant changes at the genus level (including Lactococcus and Staphylococcus) among G1 compared to $\mathrm{G} 3$ subjects.

Conclusion: Early exposure to PCV7 does not reduce pneumococcal carriage but alters the carriage of vaccine serotypes and impacts the development of the microbiome which has important implications for the long-term effectiveness of PCV.

No conflict of interest 


\title{
WHAT IS THE BEST METHOD FOR SPECIMEN TRANSPORT TO ACHIEVE OPTIMAL PNEUMOCOCCUS AND NONTYPABLE HAEMOPHILUS INFLUENZAE VIABILITY?
}

\author{
J.Y.R. Lai ${ }^{1}$, R. Marsh ${ }^{1}$, H. Smith-Vaughan ${ }^{1}$ \\ ${ }^{1}$ Child Health, Menzies School of Health Research, Darwin, Australia
}

Background and Aims: Bacterial viability can be affected by storage and transport conditions prior to processing. In remote settings, temporary storage conditions before transport to a laboratory may be necessary. This study aims to determine optimal storage conditions for maintaining pneumococcus and nontypable Haemophilus influenzae (NTHi) viability prior to processing or long-term storage at $-80^{\circ} \mathrm{C}$.

Methods: The study was conducted in two phases: i) a controlled in vitro experiment using four pneumococcal serotypes and one NTHi to determine bacterial survival at varying concentrations in different storage conditions (liquid nitrogen (LN2) vapour shipper, dry ice, domestic freezer, domestic fridge, ice bricks, crushed ice) prior to $-80^{\circ} \mathrm{C}$ storage; ii) investigating the impact of the different storage conditions on the viability of pneumococcus and NTHi using nasopharyngeal (NP) swabs.

Results: The in vitro experiments showed no significant difference between survival of different pneumococcal serotypes subject to different storage conditions up to 24 hours after inoculation. Placement of isolates directly into a LN2 vapour shipper led to approximately $30 \%$ loss of NTHi viability; findings suggest that freezing at $-20^{\circ} \mathrm{C}$ may be necessary before LN2 shipper storage.

Conclusion: Transport conditions for NP specimens need to account for the storage and transport needs of individual bacteria of interest, and will depend on time required for temporary storage. For studies requiring a LN2 vapour shipper, specimens may need prior freezing at $-20^{\circ} \mathrm{C}$ to maximise viability of NTHi.

No conflict of interest

ISPPD-0334

Pneumococcal Colonization and Carriage

\section{CARRIAGE OF PNEUMOCOCCUS AND NON-TYPEABLE HAEMOPHILUS INFLUENZAE IN CHILDREN VACCINATED WITH EITHER 7-VALENT OR 10-VALENT PNEUMOCOCCAL CONJUGATE VACCINES}

A. Leach ${ }^{1}$, C. Wigger ${ }^{1}$, H.C. Smith-Vaughan ${ }^{1}$, K. Hare ${ }^{1}$, R. Andrews ${ }^{1}$, P.S. Morris ${ }^{1}$

${ }^{1}$ Child Health Division, Menzies School of Health Research, Darwin, Australia

Background and Aims: Otitis media (OM) remains a significant health and social issue for Australian Indigenous children living in remote communities; almost all (90\%) have some form of OM and around $20 \%$ have chronic suppurative OM. In late 2009, the Northern Territory childhood vaccination schedule replaced 7-valent pneumococcal conjugate vaccine (PCV7) with 10-valent pneumococcal Haemophilus influenzae protein D conjugate vaccine (PHiD-CV10). Regular surveillance was undertaken to monitor nasopharyngeal (NP) carriage and OM prevalence.

Methods: Children were eligible if they were resident in a participating remote Indigenous community between September 2008 and December 2012 and were 0 to $<6$ years of age. This analysis compares NP carriage in PCV7and PHiD-CV10-vaccinated children ( $>=2$ doses $)<36$ months of age.

Results: Carriage of either pneumococcus (Spn) or non-typeable $\mathrm{H}$. influenzae (NTHi) was 90\%; around 35\% pneumococci were penicillin non-susceptible and $16 \%$ azithromycin resistant.

Conclusion: Pneumococcal and NTHi carriage is almost universal in young Indigenous children, regardless of vaccine type. Replacement non-susceptible non-PCV types now dominate. Post-booster analyses are warranted. Broader vaccine protection from an early age is urgently needed.

\begin{tabular}{|l|l|l|}
\hline Vaccine & PCV7 & PHiD-CV10 \\
No. swabs/No. children & $304 / 323$ & $402 / 444$ \\
\hline Mean age (months) & $\sim 17.5$ & $\sim 18$ \\
\hline Spn & $74 \%$ & $82 \%$ \\
\hline NTHi & $75 \%$ & $78 \%$ \\
\hline Both Spn and NTHi & $63 \%$ & $68 \%$ \\
\hline Neither Spn nor NTHi & $14 \%$ & $8 \%$ \\
\hline Penicillin non-susceptible Spn & $32 \%$ & $41 \%$ \\
Azithromycin resistant Spn & $19 \%$ & $14 \%$ \\
\hline Dominant serotypes in rank order (bold =non- & $\mathbf{1 6 F}, \mathbf{1 9 A}, \mathbf{1 1 A}, 6 \mathrm{C}, \mathbf{1 9 F}, \mathbf{2 3 B}$, & $\mathbf{1 6 F}, \mathbf{1 9 A}, \mathbf{1 1 A}, 6 \mathrm{C}, \mathbf{1 0 A}$, \\
\hline susceptible) & $\mathbf{1 0 A}, \mathbf{2 3 F}$ & $\mathbf{2 3 F}, \mathbf{2 3 B}$ \\
\hline PCV7 serotypes & $12 \%$ & $9 \%$ \\
\hline PHiD-CV10 serotypes & $15 \%$ & $11 \%$ \\
\hline
\end{tabular}




\section{PREVENAR AND SYNFLORIX IN A COMBINATION SCHEDULE FOR PREVENTION OF EARLY OTITIS MEDIA: PRELIMINARY SECONDARY OUTCOMES OF PREV-IX_COMBO, A RANDOMISED CONTROLLED TRIAL}

K. Mulholland ${ }^{1}$, M. Santosham ${ }^{2}$, P. Torzillo ${ }^{3}$, N. Brown ${ }^{4}$, P. Mclntyre ${ }^{5}$, H.C. Smith-Vaughan ${ }^{6}$, S. Skull ${ }^{7}$, A. Balloch ${ }^{8}$, R. Andrews ${ }^{9}$, J. Carapetis $^{10}$, V. Krause ${ }^{11}$, P.S. Morris ${ }^{9}$

${ }^{1}$ Global Health Division, Menzies School of Health Research, Darwin, Australia; ${ }^{2}$ Bloomberg School of Public Health, Johns Hopkins, Baltimore, USA; ${ }^{3}$ Respiratory Medicine, Royal Prince Alfred Hospital, Sydney, Australia; ${ }^{4}$ Indigenous Health and Education, University of Woolongong, Sydney, Australia; ${ }^{5}$ Policy and Surveillance, National Centre for Immunisation Research and Surveillance, Sydney, Australia; ${ }^{6}$ Respiratory Pathogens Laboratory, Menzies School of Health Research, Darwin, Australia; ${ }^{7}$ School of Paediatrics and Child Health, University of Western Australia, Perth, Australia; ${ }^{8}$ Research Group for Allergy \& Immune Disorders, Murdoch Childrens Research Institute, Melbourne, Australia; ${ }^{9}$ Child Health Division, Menzies School of Health Research, Darwin, Australia; ${ }^{10}$ Director, Telethon Institute for Child Health Research, Perth, Australia; ${ }^{11}$ Centre for Disease Control, Northern Territory Department of Health, Darwin, Australia

Background: Otitis media (OM) in high risk children is caused by co-infection with pneumococcus (Spn) and nontypeable Haemophilus influenzae (NTHi). Prevenar7 (PCV7) has not had a significant impact on tympanic membrane perforation (TMP) rates of $20 \%$ by 6 months in Aboriginal children in the Northern Territory. An early, mixed vaccine schedule which maximises potential activity against diverse pathogens may be beneficial.

Methods: A randomised controlled trial (RCT) of i) 10 -valent pneumococcal $H$. influenzae protein D conjugate vaccine Synflorix (PHiD-CV10) versus ii) 13-valent pneumococcal conjugate vaccine (PCV13) (at 2,4,6 months) versus iii) an early combination schedule of PHiD-CV10 at 1,2 and 4 months with additional PCV13 at 6 months. Allocation is concealed and primary outcome (immunogenicity at 7 months of age) is assessed blind. Estimated sample size is 339. Nasopharyngeal (NP) carriage and OM are secondary outcomes.

Results: Eighty seven infants have been randomised, 3 have withdrawn. Overall prevalence of OM, TMP and NP carriage at 1, 2, 4, 6 and 7 mo is shown in Table 1.

Conclusion: High risk populations may benefit from variation of standard vaccine schedules but implementation challenges necessitate high quality evidence to support such variations. This trial provides first RCT data on OM, immune response and carriage in PHiD-CV10 and PCV13 vaccinees, and will evaluate a novel early combined option for high risk children.

\begin{tabular}{|l|l|l|l|l|l|}
\hline Age (mo) & 1 & 2 & 4 & 6 & 7 \\
\hline$n$ & 87 & 78 & 75 & 64 & 63 \\
\hline $\begin{array}{l}\text { Any OM } \\
\text { (tympanometry) }\end{array}$ & $67 \%$ & $60 \%$ & $80 \%$ & $95 \%$ & $89 \%$ \\
\hline $\begin{array}{l}\text { TMP } \\
\text { (otoscopy) }\end{array}$ & nd & nd & $8 \%$ & $21 \%$ & $13 \%$ \\
\hline $\begin{array}{l}\text { Spn carriage } \\
\text { NTHi carriage }\end{array}$ & nd & $55 \%$ & $67 \%$ & $66 \%$ & $63 \%$ \\
\hline
\end{tabular}

Conflict of interest

\section{ISPPD-0368 \\ Pneumococcal Colonization and Carriage}

\section{CHANGING TRENDS IN PAEDIATRIC PNEUMOCOCCAL CARRIAGE IN HONG KONG}

M. Luo ${ }^{1}$, V. Liyanapathirana ${ }^{1}$, I. Ang ${ }^{1}$, S.A. Lui ${ }^{1}$, H. Ma ${ }^{1}$, A. Wong ${ }^{1}$, E.A.S. Nelson ${ }^{2}$, M. Ip ${ }^{1}$

${ }^{1}$ Microbiology, The Chinese University of Hong Kong, Shatin, Hong Kong China; ${ }^{2}$ Paediatrics, The Chinese University of Hong Kong, Shatin, Hong Kong China

Background and Aims: The impact of conjugate pneumococcal vaccines alters the dynamics of Streptococcus pneumoniae serotypes carriage necessitating surveillance of disease. Prospective surveillance of pneumococcal carriage was conducted from April 2009 in a University-tertiary care hospital in Hong Kong SAR. All paediatric patients hospitalized with with respiratory illness and/or fever with a nasopharygeal aspiration performed were included.

Methods: Nasopharyngeal aspirates (NPA) collected in to a modified transport medium were analyzed using routine microbiological procedures. Micro-broth based methodology (CLSI) was used to obtain the MIC of the isolates while sequential-multiplex PCR (CDC/Atlanta) were used for serotyping.

Results: Data was analyzed for 6-monthly intervals; April-September and October-March. Of the 10,909 NPA samples, $1359(12.5 \%)$ yielded S. pneumoniae. Over the 7 time periods, a significant reduction in the pneumococcal isolation rates ( $p<0.007$, Chi square for trend) was identified. Of the 1291 isolates available for further analysis, the commonest serotypes were $19 \mathrm{~F}(14 \%), 6 \mathrm{~B}$ and $6 \mathrm{C}$ (9.8\% each).Percentage of serotypes/ groups included in the PCV 7 ranged from 50.2\% in April- Sept 2009 to 24.2\% in Oct-2011- March 2012, whilst those included in the PCV13 ranged from $66.5 \%$ to $40.4 \%$ for the same time periods ( $p<0.005$, Chi square for trend). The overall cefotaxime non-susceptibility rate at meningitis and non-meningitis breakpoints (CLSI) were $29.4 \%$ and $12.9 \%$ respectively. 
Conclusion: Pneumococcal carriage rates among hospitalized children showed a significant reduction with the introduction of pneumococcal vaccination in Hong Kong. However, continuous monitoring is needed to identify long term trends that may come with serotype replacement.

No conflict of interest

ISPPD-0428

Pneumococcal Colonization and Carriage

\title{
STREPTOCOCCUS PNEUMONIAE, HAEMOPHILUS INFLUENZAE AND STAPHYLOCOCCUS AUREUS NASOPHARYNGEAL COLONIZATION IN HIV-INFECTED AND -UNINFECTED INFANTS VACCINATED WITH PNEUMOCOCCAL CONJUGATE VACCINE
}

\author{
S.A. Madhi ${ }^{1}$, A. Izu ${ }^{2}$, M.F. Cotton ${ }^{3}$, A. Violari ${ }^{4}$, N. van Niekerk ${ }^{4}$, A. von Gottberg ${ }^{1}$, K.P. Klugman ${ }^{2}$, P. Adrian ${ }^{2}$
}

${ }^{1}$ Center for Respiratory and Meningitis Diseases, National Institute for Communicable Diseases; Sandringham Johannesburg, South Africa;

${ }^{2}$ MRC: Respiratory and Meningeal Pathogens Research Unit, University of the Witwatersrand, Bertsham Johannesburg, South Africa; ${ }^{3}$ KIDSClinical Research Unit, Stellenbosch University, Stellenbosch Western Cape, South Africa; ${ }^{4}$ Perinatal HIV Research Unit, University of the Witwatersrand, Bertsham Johannesburg, South Africa

Background: Streptococcus pneumoniae, Haemophilus influenzae and Staphylococcus aureus are potentially pathogenic bacteria which colonize the nasopharynx (NP), with HIV-infected children at greater risk for invasive disease. Pneumococcal conjugate vaccine (PCV) reduces NP-colonization by the vaccine-serotypes, but may affect the equilibrium of NP-colonization by other bacteria in healthy children. We compare the bacterial NP-colonization between HIV-infected $(n=321)$ and HIV-uninfected $(n=243)$ children vaccinated with 7-valent PCV at 6, 10 and 14 weeks of age.

Methods: Children in Soweto, South Africa, had NP-swabs for bacterial culture taken prior to the three PCV-doses (Visits 1-3) and at 19.5, 39, 47 and 67 weeks of age (Visits 4-7). Swabs were cultured for bacteria by standard methods and pneumococcal serotyping done by the Quellung method.

Results: Prevalence of vaccine-serotype colonization was similar between HIV-infected and HIV-uninfected children, except Visit-6 ( $p=0.019)$; Figure. Overall pneumococcal (Visits 1-4) and non-vaccine serotype (Visits 1-5) colonization were lower in HIV-infected children. HIV-infected children had lower prevalence of $S$. aureus colonization at Visit-1 ( $p<0001)$, but higher prevalence from Visit-4 onward $(p<0.05)$; whereas H. influenzae colonization was greater among HIV-uninfected from Visit-2 $(p<0.05)$.

Conclusion: Although HIV-infected children had a similar prevalence of vaccine-serotype colonization compared to HIV-uninfected children, they had a lower prevalence of non-vaccine serotype and $H$. influenzae colonization, but higher prevalence of $S$. aureus colonization.

Figure: S. pneumoniae, S. aureus and H. influenzae colonization in HIV-infected and -uninfected children.

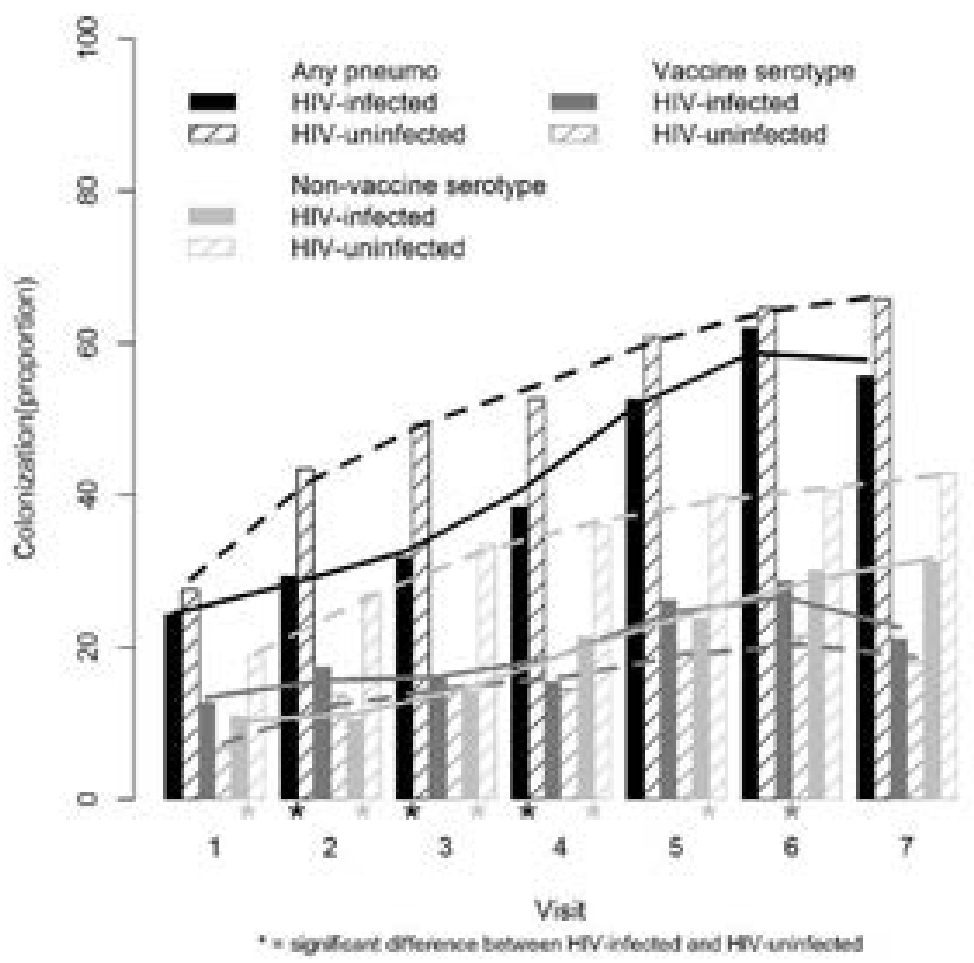




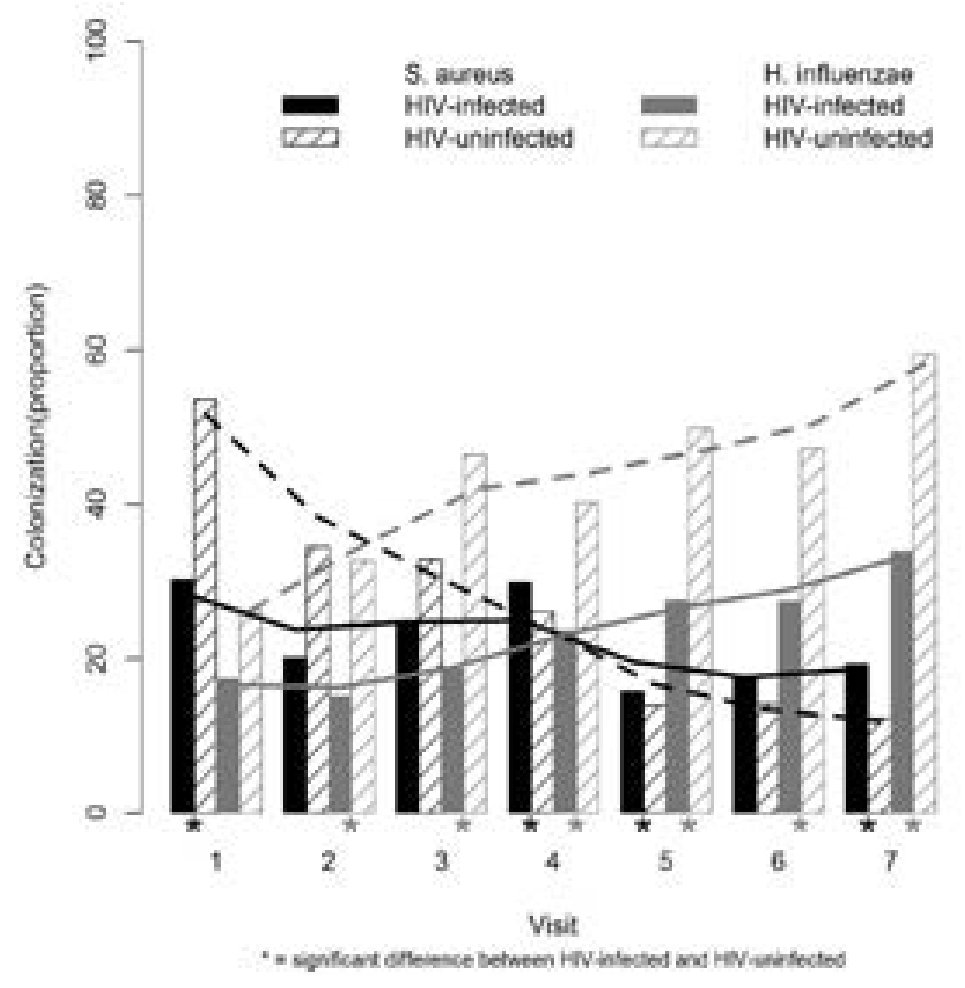

Conflict of interest

ISPPD-0314

Pneumococcal Colonization and Carriage

\section{EFFICACY OF PNEUMOCOCCAL CONJUGATE VACCINE (PREVENAR13 ${ }^{\circledR}$ ) IN PREVENTING ACQUISITION AND CARRIAGE OF PNEUMOCOCCAL VACCINE SEROTYPES IN TANZANIAN CHILDREN WITH HIV/AIDS}

G. Makenga ${ }^{1}$, G. Mtove ${ }^{1}$, A. Mziray ${ }^{1}$, V. Bwana ${ }^{1}$, W. Kisinza ${ }^{1}$, J. Mjema ${ }^{2}$, B. Amos ${ }^{3}$, R. Mallahiyo ${ }^{4}$, L. Antony ${ }^{5}$, L. Gilbert $^{6}$, S. Oftadeh ${ }^{7}$, K. Yin ${ }^{8}$, D. Shingadia ${ }^{9}$, H. Reyburn ${ }^{10}$, R. Booy ${ }^{11}$

${ }^{1}$ Clinical Trial, National Institute for Medical Research (NIMR), Tanga, Tanzania; ${ }^{2}$ Diana Centre, MuhezaTeule Hospital, Tanga, Tanzania; ${ }^{3}$ Laboratory, MuhezaTeule Hospital, Tanga, Tanzania; ${ }^{4}$ Medical Officer, MuhezaTeule Hospital, Tanga, Tanzania; ${ }^{5}$ MBBS, University of Sydney, Sydney, Australia; ${ }^{6}$ Clinical Lead in Infection Prevention and Control, Centre for Infectious Diseases \& Microbiology-Public Health, Sydney, Australia; ${ }^{7}$ Pneumococcal Reference Lab, Centre for Infectious Diseases \& Microbiology-Public Health, Sydney, Australia; ${ }^{8}$ National Centre for Immunisation Research and Surveillance, University of Sydney, Sydney, Australia; ${ }^{9}$ Consultant in Paediatric Infectious Diseases, Great Ormond Street Hospital, Sydney, Australia; ${ }^{10}$ Clinical Research, London School of Hygiene and Tropical Medicine, London, United Kingdom; ${ }^{11} \mathrm{Clinical}$ Research, National Centre for Immunisation Research \& Surveillance of Vaccine Preventable Diseases, Sydney, Australia

Background and Aims: Up to one million children die every year due to pneumococcal disease. Streptococcus pneumoniae infections increased in both incidence and severity in persons with congenital or acquired immunodeficiency. There is lack of information on the impact of pneumococcal vaccine on children older than those covered by the Expanded Program of Immunization (EPI) vaccine schedule and in those infected with HIV, as successful immunity is dependent on mounting a sufficient immune response to the vaccine. This study aimed to (1) determine the number and proportion of children carrying new (not present at baseline) vaccine serotypes of S. pneumoniae isolated from nasopharyngeal swabs at $6 \pm 2$ months post vaccination in recipients of Prevenar $13^{\circledR}$ compared with those given Hib vaccine. (2) to determine the serum antibody response (>4-fold and geometric mean concentration) to pneumococcal vaccine serotypes at $3 \pm 1$ months after vaccination.

Method: This was a double blinded crossover randomised controlled trial of the efficacy of Prevenar $13^{\circledR}$ in preventing acquisition of nasopharyngeal carriage of S. pneumoniae among HIV infected Children aged 1-14 years. Eligible participants were randomised into Prevenar $13^{\circledR}$ or Hib vaccines each given at baseline and $3 \pm 1$ months later. Nasopharyngeal and serum samples were collected at baseline and $3 \pm 1$ months later.

Results: We have enrolled 73 under five Children, the overall pneumococcal isolation rate at baseline was $71 \%(n=$ $73)$ and $73 \%(n=68)$ after two doses. Serotyping is still done but results will be available at the conference. Conclusion: Pneumococcal colonisation is common in Children with HIV/AIDS, vaccination may reduce it but replacement colonisation may occur.

No conflict of interest 
ISPPD-0027

Pneumococcal Colonization and Carriage

\section{BACTERIAL ETIOLOGY OF ACUTE OTITIS MEDIA IN CHILDREN: THE ROLE OF STREPTOCOCCUS PNEUMONIAE AND CORRELATION WITH NASOPHARYNGEAL COLONIZATION}

T. Kulichenko ${ }^{1}$, A. Ivanenko ${ }^{2}$, T. Polunina ${ }^{3}$, N. Alyabieva ${ }^{4}$, O. Ponomarenko ${ }^{4}$, A. Lazareva ${ }^{4}$, L. Katosova ${ }^{4}$, N. Mayanskiy ${ }^{4}$, L. Namazova-Baranova ${ }^{5}$

${ }^{1}$ Pediatric Emergency, Scientific Centre of Children Health of Russian Academy of Medical Science, Moscow, Russia; ${ }^{2}$ ENT, Morozovskaya Children's Hospital, Moscow, Russia; ${ }^{3}$ ENT, Scientific Centre of Children Health of Russian Academy of Medical Science, Moscow, Russia; ${ }^{4}$ Laboratory Department, Scientific Centre of Children Health of Russian Academy of Medical Science, Moscow, Russia; ${ }^{5}$ Prophylactic Pediatrics, Scientific Centre of Children Health of Russian Academy of Medical Science, Moscow, Russia

Background and Aims: Acute otitis media (AOM) is one of the most common infections in children. There are several bacterial species among principal AOM-associated pathogens but the leading role is usually attributed to Streptococcus pneumoniae. This study was performed to clarify etiology of bacterial AOM and compare it with nasopharyngeal colonization.

Methods: We examined middle ear fluid (MEF) obtained from 310 patients with AOM who were $\leq 60$ month old (age median, 28 months) at five pediatric hospitals in Moscow (Russia). In parallel, in 305 patients nasopharyngeal swab was collected. Serotyping was performed by specific antisera from Statens Serum Institute (SSI) (Denmark).

Results: Culture-positive AOM was observed in 39\% (121/310) of patients. S. pneumoniae was isolated in 55\%, Staphylococcus pyogenes in $22 \%$, Staphylococcus aureus in $14 \%$, Haemophilus influenzae in $12 \%$, and Moraxella catarrhalis in $8 \%$ of culture-positive patients. Among $67 \mathrm{~S}$. pneumoniae MEF isolates, 15 different serotypes were identified but 6 leading serotypes contributed to $>75 \%$ of the distribution $(19 \mathrm{~F}, n=17 ; 3, n=12 ; 6 \mathrm{~B}, n=9 ; 14, n=5$; $19 \mathrm{~A}, n=5 ; 23 \mathrm{~F}, n=4)$. MEF and nasopharyngeal serotypes were the same in $>90 \%(32 / 35)$ of cases. In a patient with $\mathrm{AOM}$, isolation of $S$. pneumoniae or S. pyogenes from the nasopharynx increased the probability of AOM of that particular etiology to $60 \%$ or $75 \%$, respectively. S. pneumoniae isolates displayed reduced susceptibility or resistance to penicillin (29\%), macrolides (26\%), co-trimoxazole (50\%).

Conclusion: The leading bacterial pathogen in children with AOM was $S$. pneumoniae. Available pneumococcal polysaccharide conjugate vaccines (PCV) cover from $>90 \%$ (PCV-13) to 61\% (PCV-7 and PCV-10) of the AOMassociated serotypes.

No conflict of interest

ISPPD-0026

Pneumococcal Colonization and Carriage

\section{SEROTYPES AND ANTIBIOTIC RESISTANCE OF NONIVASIVE STREPTOCOCCUS PNEUMONIAE CIRCULATING IN PEDIATRIC HOSPITALS IN MOSCOW (RUSSIA)}

N. Mayanskiy ${ }^{1}$, N. Alyabieva ${ }^{1}$, O. Ponomarenko ${ }^{1}$, A. Lazareva ${ }^{1}$, L. Katosova ${ }^{1}$, A. Ivanenko ${ }^{2}$, T. Kulichenko ${ }^{3}$, L. Namazova-Baranova ${ }^{4}$

${ }^{1}$ Laboratory dept., Scientific Centre of children health of Russian Academy of medical science, Moscow, Russia; ${ }^{2}$ ENT, Morozovskaya Children's Hospital, Moscow, Russia; ${ }^{3}$ Pediatric Emergency, Scientific Centre of children health of Russian Academy of medical science, Moscow, Russia; ${ }^{4}$ Prophylactic Pediatrics, Scientific Centre of children health of Russian Academy of medical science, Moscow, Russia

Background and Aims: Pneumococcal infections remain a major medical problem associated with high morbidity and mortality. Moreover, the resistance of Streptococcus pneumoniae to conventional antibiotics is constantly growing. Implementation of pneumococcal conjugate vaccines (PCVs) has dramatically reduced the incidence of the vaccine type-associated pneumococcal diseases in many countries. However, information on seroepidemiology of $S$. pneumoniae in Russia is limited.

Methods: We performed serotyping and antibiotic susceptibility testing on 867 noninvasive pneumococcal isolates prospectively collected in 2009-2013 from children (median age 3.5 years) who sought medical care at five pediatric hospitals in Moscow. The isolates were recovered from nasopharynx (71.2\%), middle ear fluid (14.3\%), and lower respiratory tract specimens (13.6\%).

Results: In total, 45 different serotypes were identified. The leading serotypes included serotypes $19 \mathrm{~F}(21.6 \%)$, 6B (12.8\%), 23F (10.1\%), $14(9.1 \%), 6 \mathrm{~A}(8.3 \%)$, and 3 (7.6\%). The frequency of the PCV-7, -10 , and -13 serotypes was $58.2 \%, 59.8 \%, 78 \%$, respectively. The rate of the multidrug resistant pneumococci (MDR, i.e. resistant to $>=3$ antimicrobials) was $21.5 \%$. The majority of the MDR isolates had serotype 6B, 14, 19A, and 19F. Penicillin nonsusceptibility displayed $27.5 \%$ of the isolates. The resistance rate to erythromycin was $26.1 \%$. Among the examined erythromycin-resistant strains, 54\% had erm(B) gene and $13 \%$ had mef gene as a single resistance determinant, whereas both determinants were found in $31 \%$ of these strains.

Conclusions: Our data predict a good coverage of the circulating S. pneumoniae by the PCVs and could be useful for evaluating serotype distribution in support of PCV introduction in Russia.

No conflict of interest 
ISPPD-0313

Pneumococcal Colonization and Carriage

\title{
KINETICS OF ANTIBODIES AGAINST MAJOR PNEUMOCOCCAL PROTEINS IN MOTHER AND CHILD
}

\author{
A.L. Mendy ${ }^{1}$, O.A. Aderonke ${ }^{1}$, J.S. Sutherland ${ }^{1}$, M.O. Ota ${ }^{2}$ \\ ${ }^{1}$ Vaccinology, Medical Research Council, Banjul, Gambia; ${ }^{2}$ WHO, WHO AFRO, Brazzaville, Gambia
}

Background: Invasive pneumococcal diseases (IPD) are responsible for almost 1 million deaths per year among children under five years of age. Streptococcus pneumoniae, one of the main causative agents, has over 90 serotypes yet the current conjugate vaccine only includes 13 serotypes (PCV13). Pneumococcal proteins are conserved across all serotypes and therefore hold potential as vaccine candidates.

Aims: This study aimed to investigate the transfer of antibodies against major pneumococcal antigens from pregnant mothers to their infants, and to correlate antibody concentrations with nasopharyngeal carriage (NPC). Methods: We analysed plasma from mother's (up to 3 months post-birth) and infants (cord blood only) to determine antibody concentrations and avidity to recombinant pneumolysin ( $r$ Ply), Pneumococcal surface protein A (PspA) and choline-binding protein A (CbpA) using an in-house IgG ELISA.

Results: Antibody transfer was antigen-dependent with significantly higher levels of antibodies against PspA and CbpA in mothers compared to cord blood but similar levels for rPly. PspA and CbpA were better transferred than rPly from mother to child ( $p=0.0001$ and $p=0.0024$ respectively). Antibodies to PspA and CbpA but not rPly were retained up to 3 months in mothers with no significant difference in the avidity index at any time-point.

Conclusion: Our pilot data shows that transfer of antibodies against major pneumococcal proteins from mother to child is antigen-dependent. Further work will explore the relationship with NPC. This information together with current studies on infant antibody development will help to elucidate levels of antibodies required for protection against NPC and invasive disease.

No conflict of interest

ISPPD-0184

Pneumococcal Colonization and Carriage

\section{STREPTOCOCCUS PNEUMONIAE NOVEL ADHESINS AND THEIR TARGET MOLECULES ON NEURAL CELLS}

\author{
Y. Mizrachi Nebenzahl ${ }^{1}$, M. Shagan ${ }^{1}$, A. Perlman ${ }^{1}$, A. Brieman ${ }^{2}$, R. Dagan ${ }^{3}$ \\ ${ }^{1}$ Pediatric infectious disease unit Soroka University Medical Center and The Shrtaga Segal Department of Microbiology Immunology and Ge- \\ netics, Ben Gurion University of The Negev, Beer-Sheva, Israel; ${ }^{2}$ The Shrtaga Segal Department of Microbiology Immunology and Genetics, \\ Ben Gurion University of The Negev, Beer-Sheva, Israel; ${ }^{3}$ Pediatric infectious disease unit Soroka University Medical Center, Ben Gurion Univer- \\ sity of The Negev, Beer-Sheva, Israel
}

Background and Aims: Nowadays, pneumococcus is the major cause for meningitis, with a mortality rate of $15 \%-40 \%$. Meningitis survivors may suffer from severe sequelae such as learning impairment, deafness, mental retardation, hydrocephalus and more. Meningitis is characterized by an acute inflammation. We hypothesize that functional alterations in the brain may result from immune independent pathways since treatment with Dexamethasone, in addition to the antibiotic treatment, did not improve therapy outcome. Previous studies in our laboratory identified a group of cell-wall adhesins and their target molecules in lung derived epithelial cells. Currently we study the possible involvement of those adhesin in Streptococcus pneumoniae interaction with brain derived neural cells.

Methods: The ability of the recombinant adhesin to interfere in S. pneumoniae adhesion to human U251 glioblastoma multiforme and NSC-34, a hybrid of mouse neuroblastoma and embryonic mouse motor neurons, cells was studied. Using confocal microscopy, the existence of the previously identified receptors in those cells was analyzed.

Results: Under conditions in which the bacteria do not affect survival, WU2 strain adhered to U251 and NSC-34 cells. rGtS, rPtsA, rFBA and rNOX were found to inhibit bacteria adhesion to the cells. Previously identified putative target molecules such as BMPER, MMRN1, Eps 1, PCDH19 and Int $\beta 4$ were found to be expressed in both cell lines. Conclusion: S. pneumoniae GtS, PtsA, FBA and NOX protein were found to mediate bacterial adhesion to neural cells. Moreover, previously identified target molecules to bacterial adhesins were found to reside in the neural cells.

No conflict of interest 
ISPPD-0341

Pneumococcal Colonization and Carriage

\title{
RISK FACTORS OF PNEUMOCOCCAL NASOPHARYNGEAL CARRIAGE PRIOR TO PCV1O INTRODUCTION IN VERY YOUNG INFANTS AND CHILDREN IN FIJI
}

E.F.G. Neal ${ }^{1}$, C. Satzke ${ }^{1}$, E. Dunne ${ }^{1}$, M. Cama ${ }^{2}$, E. Rafai ${ }^{2}$, M. Kama ${ }^{2}$, B. Porter ${ }^{1}$, T. Ratu ${ }^{3}$, K. Bright ${ }^{4}$, J. Kado ${ }^{3}$, L. Tikoduadua $^{3}$, R. Devi ${ }^{5}$, E.K. Mulholland ${ }^{1}$, F.M. Russell ${ }^{1}$

${ }^{1}$ Pneumococcal Research Group, Murdoch Childrens Research Institute, Parkville, Australia; ${ }^{2}$ Communicable Disease Division, Fiji Ministry of Health, Suva, Fiji; ${ }^{3}$ Department of Paediatrics, Fiji Ministry of Health, Suva, Fiji; ${ }^{4}$ International Health, Menzies School of Health Research, Darwin, Australia; ${ }^{5}$ Family Health Division, Fiji Ministry of Health, Suva, Fiji

Background and Aims: Fiji introduced PCV10 in 2012. Community carriage surveys are being undertaken to measure direct and indirect effects on pneumococcal nasopharyngeal carriage as part of a PCV10 evaluation. Preliminary results of risk factors for pneumococcal carriage in young infants and children in Fiji prior to PCV10 are shown.

Methods: 499 healthy 5-8 week old infants and 500 healthy 12-23 month old children participated in a crosssectional survey documenting risk factors for pneumococcal nasopharyngeal carriage. Nasopharyngeal swabs were collected using standard methods. Pneumococci in young infant swabs were identified by lytA quantitative PCR (qPCR). Dominant $\alpha$-haemolytic morphology, and an example of each morphological variant in children's swabs, were identified as pneumococci using standard methods. Risk factors were assessed by multivariate logistic regression.

Results: For young infants, univariate analysis showed rural residence and living with other young children to be significant risk factors for carriage. After controlling for confounding, only rural residence was associated with carriage (odds ratio (OR) 4.02, 95\% Cl $1.85-8.73, p<0.001$ ). For children, univariate analysis showed coryza, cough, living with $>2$ children, birth by vaginal delivery, and being indigenous Fijian to be risk factors for carriage. After controlling for confounding, carriage was associated with being indigenous Fijian $(\mathrm{OR} 2.51,95 \% \mathrm{Cl}$ $1.70-3.71, p<0.001$ ), and birth by vaginal delivery (OR 2.17, 95\% Cl 1.14-4.16, $p<0.024$ ).

Conclusion: Preliminary findings suggest carriage risk factors may differ by age. However, results are preliminary and final analyses will be presented at the meeting.

No conflict of interest

ISPPD-0173

Pneumococcal Colonization and Carriage

\section{ACQUISITION OF STREPTOCOCCUS PNEUMONIAE IN PNEUMOCOCCAL CONJUGATE VACCINE-NAÏVE HIV-EXPOSED CHILDREN}

\author{
M.C. Nunes ${ }^{1}$, B. Zeldow ${ }^{2}$, A. Violari ${ }^{3}$, S. Dittmer ${ }^{3}$, H. Cassim ${ }^{3}$, T. Thomas ${ }^{4}$, N. van Niekerk ${ }^{1}$, P.V. Adrian ${ }^{1}$, S.A. Madhi $^{1}$ \\ ${ }^{1}$ Respiratory and Meningeal Pathogens Research Unit, University of the Witwatersrand, Johannesburg, South Africa; ${ }^{2}$ Center for Biostatis- \\ tics in AIDS Research, Harvard School of Public Health, Boston, USA; ${ }^{3}$ Perinatal HIV Research Unit, University of the Witwatersrand, Johannes- \\ burg, South Africa; ${ }^{4}$ School of Pathology Microbiology and Infectious Diseases, University of the Witwatersrand, Johannesburg, South Africa
}

Pneumococcal nasopharyngeal colonization is a pre-requisite for pneumococcal disease (PD); HIV-infection increases the risk of PD. We investigated pneumococcal colonization and serotypes acquisition in perinatally HIV-exposed children. 333 HIV-infected and 491 HIV-exposed-uninfected (HEU) children who were randomized to isoniazid or placebo (P1041, TB-prophylaxis study), had 6 schedule visits between 6-42 months of age at which nasopharyngeal swabs were collected for pneumococcus serotyping. No pneumococcal vaccine was given. At study entry, $81 \%$ of the HIV-infected children were asymptomatic, $4 \%$ moderately symptomatic and $1 \%$ severely symptomatic for AIDS. HIV-infected children were less likely to be colonized with 13-valent pneumococcal conjugate vaccine (PCV13) serotypes than HEU at 6 months of age (31.5\% vs. 43.3\%; $p=0.03)$, however, no differences in colonization were observed at the other visits. Over the 36 months study period pneumococcal colonization increased in both HIV-infected and HEU children from $45.1 \%$ to $76.9 \%$ ( $p$-value for trend $<0.001$ ) and from $57.1 \%$ to $60.2 \%$, respectively ( $p$-value $=0.014)$. The rate of overall new serotype acquisition was lower among HIV-infected than HEU children (47\% vs. $52 \% ; p=0.008)$, while PCV13-serotypes acquisition was similar in both groups (33-36\%) and non-PCV13 serotypes were acquired less frequently by HIV-infected (11.0\%) compared to HEU (14\%; $p=0.009)$. Acquisition of non-typeable pneumococcus was more common in HIV-infected (4\%) than in HEU (2\%; $p=0.033)$. PCV13-serotypes new acquisition was common in our population and similar rates were observed in HIV-infected and HEU children. Despite a greater susceptibility to PD in HIV-infected children, overall and non-PCV13 acquisition rates were lower in HIV-infected compared to HIV-uninfected children.

No conflict of interest 
ISPPD-0369

Pneumococcal Colonization and Carriage

\section{ACQUISITION OF STREPTOCOCCUS PNEUMONIAE IN SOUTH AFRICAN CHILDREN VACCINATED WITH 7-VALENT PNEUMOCOCCAL CONJUGATE VACCINE (PCV7)}

M.C. Nunes ${ }^{1}$, S. Jones ${ }^{1}$, A. von Gottberg ${ }^{2}$, S.A. Nzenze ${ }^{1}$, N. van Niekerk ${ }^{1}$, A. Koen ${ }^{1}$, M. Groome ${ }^{1}$, P.V. Adrian ${ }^{1}$, S.A. Ma$\mathrm{dhi}^{1}$

${ }^{1}$ University of the Witwatersrand, Respiratory and Meningeal Pathogens Unit, Johannesburg, South Africa; ${ }^{2}$ A division of the Health Laboratory Service (NHLS), National Institute of Communicable Diseases, Johannesburg, South Africa

The 7-valent pneumoccocal conjugate vaccine (CV7) was introduced into the South African immunization program in 2009 as a three-dose schedule at 6 weeks, 14 weeks and booster at 9 months age. We studied the rate of new serotypes acquisitions in children receiving this schedule and compared it to a historical cohort of PCV-unvaccinated children. Children aged 5-8 weeks were enrolled from December/09 to April/10, while the historical cohort was enrolled in 2007. Participants had nasopharyngeal swabs done on seven occasions between one-month following the second PCV7 dose up until two years, with the same schedule undertaken among the historical cohort. Swabs were cultured for pneumococcus.

A total of 1551 nasopharyngeal swabs from 236 PCV7-vaccinated children were collected during the study, from which 1059 (68.3\%) were positive for pneumococcus. The prevalence of pneumococcal colonization peaked at 41 weeks of age $(n=175 / 228,76.8 \%)$ and ranged between $63-69 \%$ thereafter. Overall colonization by PCV7-serotypes was $21.7 \%$. During the first two years of life PCV7-vaccinated children acquired pneumococci less frequently than unvaccinated children $(46.2 \%$ vs. $55.9 \%, p<0.001)$, especially PCV7-serotypes $(11.3 \%$ vs. $25.0 \%, p<0.001)$. Non-vaccine serotypes were acquired more often by PCV7-vaccinated compared to -unvaccinated children (32.4\% vs. $27.4 \%, p=0.018$ ). Acquisition of non-typeable pneumococci was similar between PCV7-vaccinated and -unvaccinated children ( $2.6 \%$ vs. $3.5 \%, p=0.24$, respectively). This study demonstrates that the $2+1$ schedule implemented in South Africa has resulted in a reduced risk of vaccine-serotype acquisition among PCV7-vaccinated children. As such, there is expected to be reduced transmission and consequently indirect protection against pneumococcal disease among unvaccinated individuals in South Africa.

No conflict of interest

ISPPD-0272

Pneumococcal Colonization and Carriage

\section{CHANGES IN PNEUMOCOCCAL COLONIZATION IN HIV-INFECTED AND HIV-UNINFECTED MOTHER-INFANT PAIRS FOLLOWING TRANSITIONING FROM 7-VALENT TO 13-VALENT PNEUMOCOCCAL CONJUGATE VACCINE (PCV)}

\section{S.A. Nzenze ${ }^{1}$, A. von Gottberg ${ }^{2}$, T. Shiri ${ }^{1}$, L. de Gouveia² ${ }^{2}$ A. Violari ${ }^{3}$, M.C. Nunes ${ }^{1}$, S.A. Madhi ${ }^{2}$}

${ }^{1}$ Department of Science and Technology/Vaccine Preventable Diseases Unit, Respiratory and Meningeal Pathogens Unit, Johannesburg, South Africa; ${ }^{2}$ National Institute of Communicable Diseases, Respiratory and Meningeal Pathogens Unit, Johannesburg, South Africa

${ }^{3}$ University of the Witwatersrand, Perinatal HIV Research Unit, Johannesburg, South Africa

Background: Pneumococcal conjugate vaccine (PCV) immunization of children decreases their risk of nasopharyngeal acquisition of vaccine-serotypes and consequently reduces transmission to PCV-unvaccinated individuals. We explored the impact of PCV13 on pneumococcal carriage in both HIV-infected and HIV-uninfected mother-child pairs.

Methods: Two surveys were done in Soweto from May 2010 to February 2011 (Period-1) and May 2012 to April 2013 (Period-2). PCV-7 was introduced into the public immunization program for infants in April 2009 and replaced by PCV-13 from May 2011. Mother-child pairs were either both HIV-infected or HIV-uninfected. Children were stratified by age-group to $<9 \mathrm{mo}$., $9 \mathrm{mo}$. to $2 \mathrm{yrs}$ (likely to be fully-vaccinated in both Periods), $>2$ to 4 yrs (likely to be fully vaccinated in Period-2) and $>4-12$ yrs (low likelihood of PCV vaccination). Nasopharyngeal swabs were obtained for pneumococcal culture and serotyping.

Results: PCV13 serotypes colonization decreased among all age-groups in Period-2 compared to Period-1, except among HIV-infected children 0-9 months and HIV-uninfected 4-12 years. Non-vaccine serotypes either increased or remained unchanged among all age groups; Table 1

Conclusion: Our study demonstrated a temporal association of decrease in PCV13 serotype colonization among HIVinfected and HIV-uninfected females not targeted for PCV immunization. This indicates, that targeted vaccination of young infants at 6, 10 and 42 weeks of age in South Africa, is likely to confer indirect benefit in protecting adults against PCV13-serotype IPD. 
Table 1: The impact of PCV on nasopharyngeal carriage in children and mothers.

\begin{tabular}{|c|c|c|c|c|c|c|c|c|}
\hline \multirow{3}{*}{$\begin{array}{l}\text { Grove } \\
\text { Chldren }\end{array}$} & \multirow{2}{*}{\multicolumn{2}{|c|}{ Total per gowp }} & \multirow{2}{*}{\multicolumn{2}{|c|}{ All Pseumococtl }} & \multicolumn{2}{|c|}{$\begin{array}{l}\text { 13-valent vactine } \\
\text { serotyget }\end{array}$} & \multicolumn{2}{|c|}{$\begin{array}{l}\text { Non } 13 \text { valent vaccline } \\
\text { verotypes }\end{array}$} \\
\hline & & & & & \multicolumn{2}{|c|}{$n(x)$} & \multicolumn{2}{|c|}{$A(\$)$} \\
\hline & Perlod 1 & Perlod? & Perlods & Perlod? & Perlod1 & Perlods & Perlods & Perlod2 \\
\hline \multicolumn{9}{|l|}{ 0-9months } \\
\hline Hiv* & 57 & 56 & $26[45.6)$ & $20(35.7)$ & $8[140]$ & $7(12.5)$ & 1831.6 & $13 \times 232$ \\
\hline CR & & & \multicolumn{2}{|c|}{$0.66(0.31,1.41)$} & \multicolumn{2}{|c|}{$0.89(0.292 .60)$} & \multicolumn{2}{|c|}{$0.66(0.28,1.51)$} \\
\hline keV. & 173 & 340 & $99(57.2)$ & 172506 & $50(2 \times 9)$ & $47(38)$ & $51(20.5)$ & 126337.1 \\
\hline $\mathrm{Ch}$ & & & \multicolumn{2}{|c|}{$0.740,52,1.10\}$} & \multicolumn{2}{|c|}{$0.39[0.25 .0 .42]$} & \multicolumn{2}{|c|}{$1.40,095,2$.09) } \\
\hline \multicolumn{9}{|c|}{29 monthy 2 years } \\
\hline MN+ & 151 & 91 & $94(623)$ & $50(545)$ & $61(40.4)$ & $17(18.7)$ & $35(232)$ & $33(36.3)$ \\
\hline$C R$ & & & \multicolumn{2}{|c|}{$0.74(0.44,1.25)$} & \multicolumn{2}{|c|}{$0.34(0.18,0.63)$} & \multicolumn{2}{|c|}{$1.85(1.07,3.34)$} \\
\hline HV. & 257 & 44 & $173(67,3)$ & $272|69.7\rangle$ & $111(43.2)$ & $63(141)$ & $70(27.2)$ & $212(4) 3$ \\
\hline $\mathrm{CR}$ & & & \multicolumn{2}{|c|}{$0.740,4,1.02)$} & \multicolumn{2}{|c|}{$0.21[0.15,0.31)$} & \multicolumn{2}{|c|}{$2.39(1.71,2.33)$} \\
\hline \multicolumn{9}{|c|}{37 years-4yean } \\
\hline HeVt & 267 & 179 & $189(70.8)$ & $118 \mid 659)$ & $114(42.7)$ & $33(179)$ & 79.29 .61 & $88(49.2)$ \\
\hline CR & & & \multicolumn{2}{|c|}{$0.81(0.54,1.22)$} & \multicolumn{2}{|c|}{$0.29(0.19,0.46)$} & \multicolumn{2}{|c|}{$2.30(1.55,3.41)$} \\
\hline HNV. & 182 & 168 & $1276.8)$ & $100(64.3)$ & $75(41.2)$ & $22(131)$ & $5 \mathrm{~kg}(919)$ & $86(512)$ \\
\hline $\mathrm{Ca}$ & & & \multicolumn{2}{|c|}{$0.750 .51,1.24)$} & \multicolumn{2}{|c|}{$0.22(0.13,0.37)$} & \multicolumn{2}{|c|}{$2.27(1.47,351)$} \\
\hline \multicolumn{9}{|c|}{ S-4yeart-13years } \\
\hline $\mathrm{MIN}+$ & 233 & 303 & $173(74.2)$ & $195(64.4)$ & $89(38.2)$ & $71(23.4)$ & $94(403)$ & $125 / 41.3)$ \\
\hline$C R$ & & & 0.6310 & $2,0.931$ & 0.500 & a.73) & 1.03 & $2,1.69$ \\
\hline HV. & 91 & 63 & $45(53.8)$ & $29(46.0)$ & $21(23.1)$ & $11(175)$ & $30 \times 3301$ & $19(30.2)$ \\
\hline Cn & & & 0.730 & $7,1.46)$ & Q710 & $1.69)$ & 088 & $1,1.85$ \\
\hline Mothers & & & & & & & & \\
\hline MNt & 207 & 616 & $14(204)$ & $8 ;(140)$ & $61(8.6)$ & $29(4.7)$ & $87(12.3)$ & $57(9.3)$ \\
\hline CR & & & 0.6410. & 3.0 .85 & 0.521 & 0.83) & 0.73 & $1,1.03$ \\
\hline HiV. & 669 & 918 & $65(9.7)$ & $87 \mid 24$ & $365.4)$ & $182.0)$ & $30(45)$ & $69(75)$ \\
\hline$C n$ & & & $0.95 \%$ & 1.37 & 0.351 & 0.62) & 1.7. & $1,2.691$ \\
\hline
\end{tabular}

No conflict of interest

ISPPD-0129

Pneumococcal Colonization and Carriage

\section{PREVALENCE OF RESPIRATORY SYMPTOMS, VIRUSES AND BACTERIA IN A REMOTE ABORIGINAL COMMUNITY IN CENTRAL AUSTRALIA}

K. O'Grady ${ }^{1}$, P.J. Torzillo², D.M. Whiley ${ }^{3}$, T.P. Sloots ${ }^{3}$, S.B. Lambert ${ }^{3}$

${ }^{1}$ Queensland University of Technology, Queensland Children's Medical Research Institute, Herston, Australia; ${ }^{2}$ Sydney University, Sydney Medical School, Sydney, Australia; ${ }^{3}$ University of Queensland, Queensland Children's Medical Research Institute, Herston, Australia

Background and Aims: Central Australian Aboriginal communities have amongst the highest reported rates of respiratory infections worldwide. There are however no community level data on the prevalence of respiratory symptoms and respiratory pathogens that may be associated with those symptoms. Our aim was to address this knowledge gap.

Methods: We convenience sampled every individual who presented for any reason to a remote community clinic over a three week period in a non-epidemic respiratory season. Demographic and clinical data were obtained. Anterior nasal swabs were collected and tested for adenovirus, respiratory syncytial virus (RSV), influenza virus, parainfluenza viruses (PIVs) types 1,2 3, human metapneumovirus (hMPV), rhinoviruses (RVs), coronaviruses (hCoV) (OC43, 229E,NL63 + HKU1), bocavirus, KI and WU polymaviruses (KI and WUPyV), Streptococcus pneumoniae, Haemophilus influenzae, Moraxella catarrhalis, Staphylococcus aureus (including Methicillin-resistant Staphylococcus aureus (MRSA)), and Bordatella pertussis using real-time PCR methods.

Results: 140 participants were enrolled, contributing 153 study visits and 150 nasal specimens; $21 \%$ were aged < 5 years. A respiratory symptom was present in 74 (48.4\%) of all episodes. At least one virus was present in $50 \%$ of episodes with a specimen, at least one bacterium in $80 \%$ of episodes and viral-bacterial coinfection was present in $28.7 \%$. Respiratory symptoms were 2.4 times $(95 \% \mathrm{Cl} 1.4-4.3)$ more likely in episodes of viral-bacterial coinfection than those without.

Conclusions: The prevalence of both symptoms and pathogens are high this population. Interventions to reduce the burden of respiratory disease in this population will require community wide approaches to reduce infection and carriage in both children and adults.

No conflict of interest 
ISPPD-0213

Pneumococcal Colonization and Carriage

\begin{abstract}
VIRUSES AND BACTERIA ASSOCIATED WITH HOSPITALISED, RADIOLOGICALLY CONFIRMED PNEUMONIA IN ABORIGINAL CHILDREN AGED LESS THAN FIVE YEARS IN CENTRAL AUSTRALIA
\end{abstract}

\author{
K. O'Grady ${ }^{1}$, P.J. Torzillo' ${ }^{2}$, P. Valery ${ }^{3}$, H. Smith-Vaughan ${ }^{3}$, T.P. Sloots ${ }^{4}$, A.R. Ruben ${ }^{5}$, D. Taylor-Thomson ${ }^{3}$, A.B. Chang ${ }^{1}$ \\ ${ }^{1}$ Queensland Children's Medical Research Institute, Queensland University of Technology, Herston, Australia; ${ }^{2}$ Sydney Medical School, Syd- \\ ney University, Sydney, Australia; ${ }^{3}$ Menzies School of Health Research, Charles Darwin University, Darwin, Australia; ${ }^{4}$ Queensland Children's Med- \\ ical Research Institute, The University of Queensland, Herston, Australia; ${ }^{5}$ NT Clinical School, Flinders University, Cairns, Australia
}

Background and Aims: Aboriginal children in remote regions of Central Australia have the highest yet reported rates of hospitalised, World Health Organisation (WHO) defined, radiologically-confirmed pneumonia. We aimed to describe the respiratory viruses and bacteria detected in deep nasal swabs associated with hospitalised pneumonia in these children.

Methods: Datasets from two studies of hospitalised pneumonia in Central Australian Aboriginal children aged less than 5 years were combined. Chest xrays were assessed for consolidation according to the WHO protocol and by a paediatric pulmonologist (PP). Deep nasal swabs collected on admission were analysed by PCR for 6 bacteria (Streptococcus pneumoniae, Haemophilus influenzae, Staphylococcus aureus, Moraxella catarrhalis, Chlamydia pneumoniae and Mycoplasma pneumoniae) and 17 viruses (human rhinoviruses, adenovirus, human metapneumovirus, respiratory syncytial viruses $A$ and $B$, influenza viruses $A$ and $B$, parainfluenza types 1,2 and 3 , bocavirus, WU and KI polyomaviruses and human coronaviruses HUK1, OC43, nl63 and 229e).

Results: Complete clinical, radiological and laboratory data were available on 103 children. Fifty percent $(n=51)$ had PP-confirmed lobar consolidation and $28 \%(n=29)$ had consolidation as per the WHO protocol. At least one bacterium was identified in $92.2 \%$ of all children, at least one virus in $42.7 \%$ and both viruses and bacteria were detected in $39.8 \%$. There were no statistically confirmed differences between lobar and non-lobar pneumonia with respect to any organism.

Conclusion: There appears to be no clear relationship between nasal detection of respiratory viruses and bacteria and the presence of radiologically-confirmed lobar consolidation in Aboriginal children hospitalised with pneumonia.

No conflict of interest

ISPPD-0148

Pneumococcal Colonization and Carriage

\title{
PNEUMOCOCCAL CONJUGATE VACCINE PRIMES MUCOSAL IMMUNE RESPONSES FOR BOOSTING BY PNEUMOCOCCAL POLYSACCHARIDE VACCINE IN PAPUA NEW GUINEAN CHILDREN
}

T. Orami ${ }^{1}$, W. Pomat ${ }^{1}$, J. Francis ${ }^{1}$, K. Corscadden ${ }^{2}$, R. Thornton ${ }^{2}$, L. Kirkham ${ }^{2}$, P. Siba ${ }^{3}$, P. Richmond ${ }^{2}$, R. Ford ${ }^{1}$, D. Lehmann ${ }^{4}$

${ }^{1}$ Infection and Immunity, Papua New Guinea Institute of Medical Research, Goroka, Papua New Guinea; ${ }^{2}$ Vaccine Trials Group, School of Paediatrics and Child Health University of Western Australia, Perth, Australia; ${ }^{3}$ Director, Papua New Guinea Institue of Medical Research, Goroka, Papua New Guinea; ${ }^{4}$ Division of Population Sciences, Telethon Institute for Child Health Research University of Western Australia, Perth, Australia

Introduction: Upper respiratory tract mucosal surfaces are primary sites of pneumococcal infections. Pneumococcal vaccines may induce production of salivary IgA and IgG. Mucosal antibody responses to pneumococcal vaccines were investigated in a pneumococcal conjugate vaccine (PCV7) trial in Papua New Guinea.

Methods: Children were randomized to receive PCV7 in a 0-1-2 (neonatal) or 1-2-3-month (infant) schedule or no PCV7. All children received pneumococcal polysaccharide vaccine (PPV) at age 9 months. Saliva was collected at ages 1, 2, 3, 4, 9, 10 and 18 months. Salivary IgA and IgG concentrations to all PCV7 serotypes and PPV serotypes 1, $3,5,7 F, 19 A$ were measured in 420 samples (60 children) using a multiplex fluorescent bead-based assay.

Results: PCV7 had little impact on salivary IgA before 9 months but geometric mean titres (GMTs) for salivary IgG to serotypes 4, 18C and 19F were higher at ages 2-4 months in PCV7 recipients than in controls. At ages 9 and 10 months, GMTs for PCV7-serotype-specific IgA were 100-1778 and 891-6607 ng/ml, respectively; in PCV7-primed children and 78-1479 and 74-3020 ng/ml in unprimed children. Equivalent ranges for salivary lgG were 38-723 and $478-12928 \mathrm{ng} / \mathrm{ml}$ in PCV-primed children and $27-214$ and $40-300 \mathrm{ng} / \mathrm{ml}$ in unprimed children. Average mean fold increases (MFIs) in PCV7-serotype-specific IgA 1 month post-PPV were 7.6, 5.5 and 2.6 in neonatal, infant and control groups, respectively and 20.4, 12.0 and 1.3 in neonatal, infant and control groups for PCV7-serotype-specific IgG.

Conclusion: PCV7 generally primes mucosal immune responses for boosting by PPV at 9 months.

No conflict of interest 


\title{
THE IMMUNE RESPONSES OF CATIONIC LIPOSOMAL POLYSACCHARIDE AGAINST STREPTOCOCCUS PNEUMONIAE
} SEROTYPE 14

\author{
S.J. Park ${ }^{1}$, K.H. Seo ${ }^{1}$, M.S. Seo ${ }^{1}$ \\ ${ }^{1} R \& D$, Center for Biopharmaceutical Research, Jeollanam-do, Korea
}

Streptococcus pneumoniae causes serious illnesses such as pneumonia, meningitis, and acute otitis media. A main antigenic determinant of $S$. pneumoniae is polysaccharide (PS), which shows a poor immunogenicity as a T cell independent antigen. In this study, we applied cationic liposome for S.pneumoniae PS and examined immune responses such as mucosal IgA, systemic IgG and T-cell response. Mucosal immunity is one of the important issues because the pathogen enters the host through the respiratory mucosa. A vaccine inducing the production of protective secretory IgA, as well as systemic IgG, would be desirable. The cationic liposomal PS showed higher respiratory mucosal IgA compared to pneumococcal conjugate vaccine. And cationic liposome brought the T-cell dependent immunity against pneumococcal polysaccharide showing considerable high T-cells proliferation compared to PS only and high Interleukin-4 (Th2 marker) production. And the cationic liposome showed a depot effect at the injection site, which could induce a prolonged presentation of the incorporated antigen. All of the results were induced with a formulation composed of PS and liposome without any additional carrier proteins. These results suggest that cationic liposomal PS could be useful for immune responses against a polysaccharidebased S. pneumoniae vaccine.

No conflict of interest

ISPPD-0171

Pneumococcal Colonization and Carriage

\section{PNEUMOCOCCAL CIPL IS A MN-DEPENDENT CHAPERONE WITHOUT AUXILIARY FACTORS}

\author{
S.S. Park ${ }^{1}$, S. Pyo ${ }^{1}$, D. Rhee ${ }^{1}$ \\ ${ }^{1}$ School of pharmacy, Sungkyunkwan University, Suwon, Korea
}

Caseinolytic protease $L(C l p L)$ is a member of HSP 100 family, found mostly in Gram-positive bacteria. Here we report the characterization of recombinant ClpL protein, a major heat shock protein (HSP) in Streptococcus pneumoniae (pneumococcus) in vitro. Previously, we characterized ClpL with 6-His tag at the N-terminal on chaperone and ATPase activities. However, N-terminal His-tag affected ClpL functions. Therefore, ClpL was rechracterized without His-tag. ClpL has ADP and ATP hydrolase activity by using either $\mathrm{Mg}^{2+}$ or $\mathrm{Mn}^{2+}, \mathrm{but}_{\mathrm{Mn}}{ }^{2+}$ stimulates ATP hydrolase activity than $\mathrm{Mg}^{2+}$. $\mathrm{Mn}^{2+}$ increases ATP hydrolase activity as well as refolding and heat aggregation prevention activities. However, ClpL does not seem to require auxiliary factors (DnaKJE system) for chaperone activity. ClpL forms hexamer in the presence of ADP, ATP, and ATP- $\gamma-\mathrm{S}$. Mutagenesis studies on the two Walker A motifs (K127A, T128A and K458A, T459A), displayed that two nucleotide binding domains are involved in chaperone and ATP hydrolase activities and hexamerizaion. Taken together, pneumococcal ClpL is a Mn-dependent auxiliary-factor independent chaperone.

No conflict of interest

ISPPD-0250

Pneumococcal Colonization and Carriage

\section{PREVALENCE OF NONVACCINE PNEUMOCOCCAL SEROTYPE NASOPHARYNGEAL CARRIAGE IN CHILDREN $<5$ YRS OF AGE IN BOSTON FOLLOWING INTRODUCTION OF PCV13}

\author{
S. Pelton ${ }^{1}$, K. Shea ${ }^{2}$, K. Hsu ${ }^{3}$, A. Loughlin ${ }^{3}$ \\ ${ }^{1}$ Pediatrics and Epidemiology, Boston Universithy Schools of Medicine and Public Health, Boston, USA; ${ }^{2}$ Epidemiology, Boston Univer- \\ sithy School of Public Health, Boston, USA; ${ }^{3}$ Pediatrics, Boston Universithy School of Medicine, Boston, USA
}

Background: Changes in prevalence of nasopharyngeal (NP) carriage of nonvaccine pneumococcal serotypes (NVST) may herald increases in IPD caused by these serotypes and potential erosion of vaccine effectiveness.

Objective: To evaluate changes in pneumococcal serotypes (All, NVST, PCV7, and PCV13 unique) in the NP following substitution of PCV13 for PCV7 in children <2 years with catch-up through 5 years.

Methods: Demographic information and NP cultures were obtained from 5,307 children $<5$ years seen in primary care between 7.1.2010 and 6.30.2013. All isolates of Streptococcus pneumoniae (SP) were serotyped by quellung reaction with type-specific antisera.

Results: The overall prevalence of SP carriage was $22.8 \%$, and was lowest during summer months. No significant changes in the carriage prevalence of All SP, NVST, or PCV7 serotypes were observed. Carriage of PCV13 unique serotypes declined over time (Figure 1). 


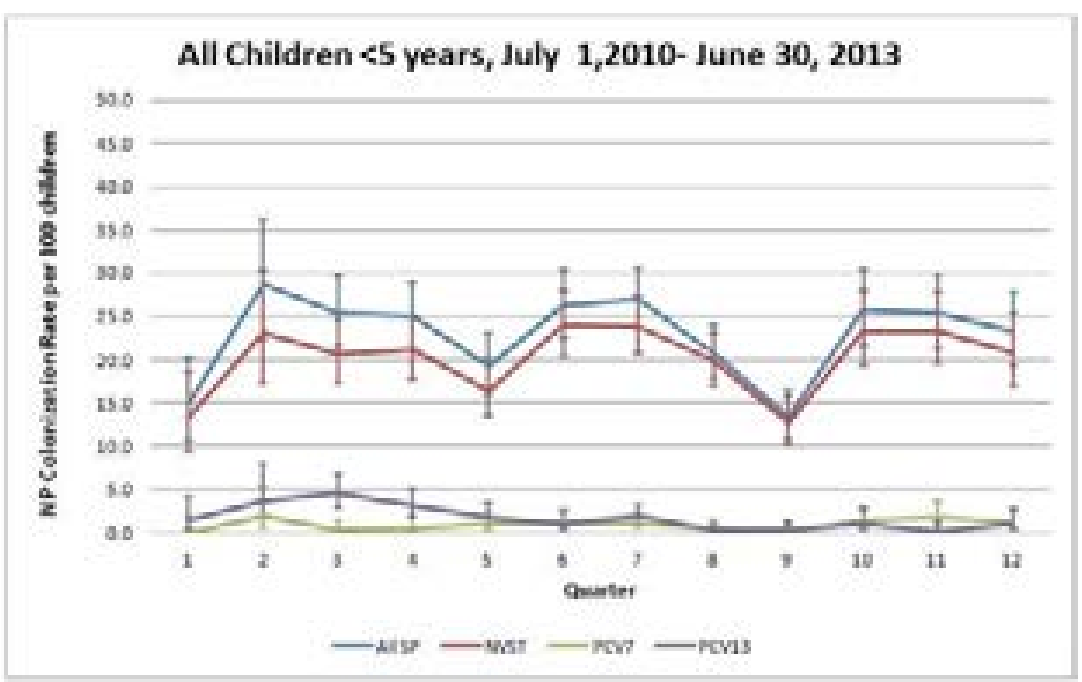

Analysis of individual serotypes demonstrated a decline in SP19A, but no change in SP6C or 3 (Figure 2)

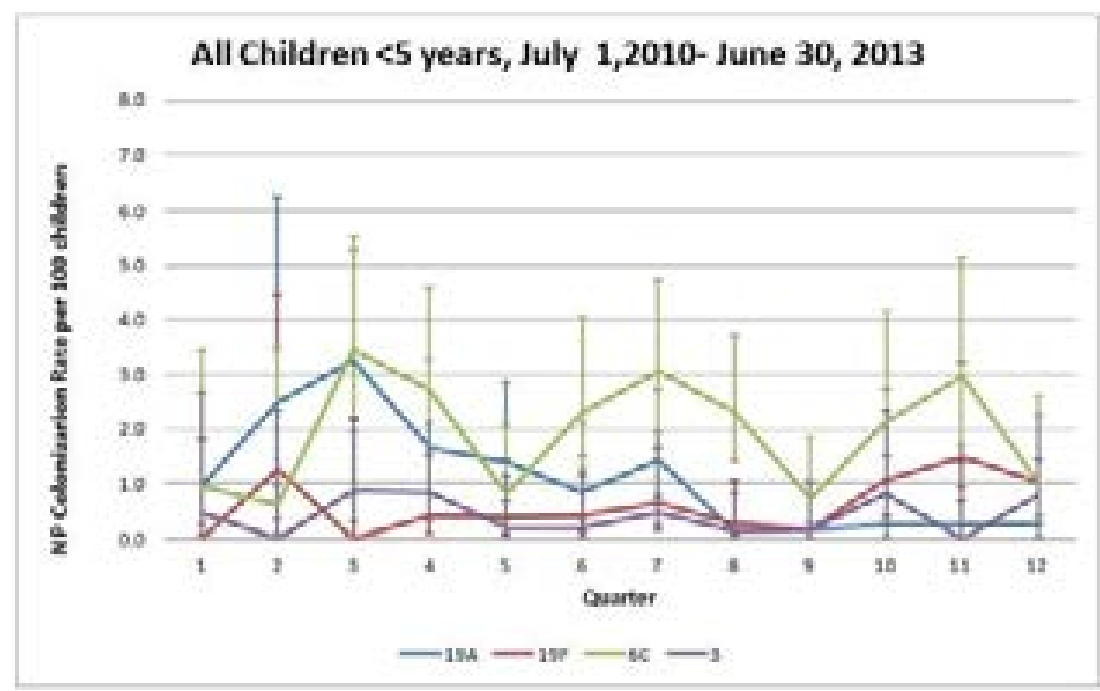

Conclusion: Following introduction of PCV13, the prevalence of overall SP colonization was unchanged. Unlike the experience with PCV7, the prevalence of overall NVST NP carriage has remained unchanged as well. PCV13 unique serotypes declined, specifically SP19A. No change in prevalence of serotype 6C or 3 has been observed.

Funding: Thrasher Research Foundation, Pfizer, Inc and Merck Vaccines during different years of the study.

Conflict of interest

ISPPD-0199

Pneumococcal Colonization and Carriage

\section{THE PREVALENCE OF STREPTOCOCCUS PNEUMONIAE NASOPHARYNGEAL CARRIAGE IN HEALTHY CHILDREN IN CENTRAL LOMBOK, INDONESIA}

\footnotetext{
A. Prayitno ${ }^{1}$, S.R. Hadinegoro ${ }^{2}$, I.G.G. Djelantik ${ }^{3}$, S.A.K. Indriyani ${ }^{3}$, N.E. Dewi ${ }^{4}$, Z. Muttaqin ${ }^{5}$, D. Safari ${ }^{6}$

${ }^{1}$ Department of Child Health, Faculty of Medicine University of Indonesia Dr Cipto Mangunkusumo hospital Jakarta Indonesia, Jakarta, Indonesia; ${ }^{2}$ Department of Child Health, Faculty of Medicine University of Indonesia Dr Cipto Mangunkusumo Hospital Jakarta Indonesia, Jakarta, Indonesia; ${ }^{3}$ Department of Pediatric, Hospital of NTB Province, Lombok, Indonesia; ${ }^{4}$ Health Department, Health Office of Central Lombok district, Lombok, Indonesia; ${ }^{5}$ Biomedical Laboratory, Hospital of NTB Province, Lombok, Indonesia; ${ }^{6}$ Pneumococcal Research, Eijkman Institute Laboratory, Jakarta, Indonesia
}

Background and Aims: Streptococcus pneumoniae is a major cause of morbidity and mortality worldwide. The pneumococcal nasopharyngeal carriage preceded and is a source of the spread of disease to others pneumococcal. Indonesia is the country with the highest incidence of pneumonia in Southeast Asia, and Lombok Island in western part of Indonesia has a highest pneumonia incidence.

Methods and Results: Pneumococcal nasopharyngeal isolate has been done in 1200 healthy children aged 2 months to 5 years of 5 Health Centre in Central Lombok, Lombok Island. On those isolates, Optochin test, PCR assay, and sensitivity to antibiotics were performed. The prevalence of $S$. pneumoniae carrier rate was $46.17 \%$, with distribution in less than 2 years of age and above were $26.8 \%$ and $48.8 \%$, respectively. Most commonly serotypes were $6 \mathrm{~A} / \mathrm{B}, 19 \mathrm{~F}, 23 \mathrm{~F}, 15 \mathrm{~B} / \mathrm{C}$, and 19A. All serotypes were susceptible to majority of antibiotics, except to cotrimoxazole (36\%). Overall of isolates belonged to strains covered by $50,1 \%$ for PCV10 and $55,3 \%$ for PCV 13 . We 
found $74.7 \%$ of the subjects exposed to passive smoking and $74.7 \%$ have pneumococcal positive isolate Conclusion: The prevalence of nasopharyngeal carriage of $S$. pneumoniae is very high and most commonly serotypes found are quite similar to those reported ten years ago in the same area. Approximately a half of carriages were covered by either PCV10 or PCV13. The serotypes distribution should be conducted to other areas to achieve the overall figure of pneumococcal pharyngeal isolate coverage by PCV

No conflict of interest

ISPPD-0378

Pneumococcal Colonization and Carriage

\title{
DEMOGRAPHIC PROFILE OF HEALTHY CHILDREN WITH NASOPHARYNGEAL COLONIZATION OF STREPTOCOCCUS PNEUMONIAE IN SOUTH INDIA
}

\author{
R. Raman $^{1}$ \\ ${ }^{1}$ Pediatrics, Kanchi Kamakoti Childs Trust Hospital, Chennai, India
}

Background: Invasive disease due to Streptococcus pneumoniae is one of the leading cause of death in children under 5 years of age. Nasopharyngeal colonization precedes the invasive disease in some children. Factors influencing nasopharyngeal colonization by $S$. pneumoniae would probably help in prevention of serious illness such as pneumonia, meningitis and septicaemia.

Aims: To study the Demographic profile of children with nasopharyngeal colonisation of S. pneumoniae.

Methods: A total of 500 children between 6 months to 5 years were included in the study. A detailed questionnaire was filled for each child to delineate the vaccination status, underlying risk factors and socioeconomic status. Nasopharyngeal swabs were obtained from children following strict protocols and were plated immediately in the appropriate culture plates.

Results: Out of the 500 children, 45 had grown S. pneumoniae in the nasopharyngeal swab culture. Majority was males, less than 2 years of age and belonged to families with a lower income. Other risk factors that were identified were average ventilation in the house hold and over crowding in a few of them. Majority had received Hib vaccine. Smoking, duration of breast feeding, attending day care and predisposing respiratory infection or use of antibiotics did not influence nasopharyngeal colonization.

Conclusion: Nasopharyngeal carriage of $S$. pneumoniae is common in male children less than 2 years of age belonging to families with a lower income.

No conflict of interest

ISPPD-0276

Pneumococcal Colonization and Carriage

\section{A PROSPECTIVE STUDY ON BACTERIAL PATHOGENS ASSOCIATED WITH ACUTE RESPIRATORY SYMPTOMS IN CHILDREN IN RURAL BALLABGARH, HARYANA (INDIA)}

V. Rastogi ${ }^{1}$, D.R. Purakayastha' ${ }^{2}$, S.K. Kapoor ${ }^{3}$, A. Wahi' ${ }^{2}$, S. Kumar ${ }^{4}$, R. Amarchand ${ }^{5}$, S. Broor ${ }^{6}$, A. Kapil ${ }^{4}$, S. Rai ${ }^{2}$, P. Misra' ${ }^{2}$ A. Krishnan ${ }^{2}$

${ }^{1}$ Program Officer, Inclen Trust International, Faridabad, India; ${ }^{2}$ Centre for Community Medicine, All India Institute of Medical Sciences, New Delhi, India; ${ }^{3}$ Professor Emeritus, Inclen Trust International, New Delhi, India; ${ }^{4}$ Department of Microbiology, All India Institute of Medical Sciences, New Delhi, India; ${ }^{5}$ Senior Program Officer, Inclen Trust International, New Delhi, India; ${ }^{6}$ Director Laboratory, Inclen Trust International, New Delhi, India

Background and Aims: Acute respiratory tract infection (ARI) is a leading cause of morbidity and mortality in young children. Streptococcus pneumoniae is the most common bacterial pathogen of pneumonia in children. We used ARI surveillance platform to identify common bacterial pathogens associated with acute respiratory symptoms in children below 10 years.

Methods: A dynamic cohort of children (population at inception 2754) in four villages of Ballabgarh was kept under weekly surveillance from Aug'12 to Aug'13. All children were screened weekly for presence of any respiratory symptom (cough, sore throat, ear ache/discharge, nasal discharge/congestion, shortness of breath/respiratory difficulty). These ARI cases were further assessed by nurses and classified into acute upper respiratory tract infection (AURI) and acute lower respiratory tract infection (ALRI) as per WHO guidelines. Age-matched healthy controls from same village were also selected. Naso-pharyngeal swabs were taken from all-ALRI, controls and $5 \%$ of AURI. Primary cultures were done on blood agar and chocolate agar at AlIMS.

Results: In 2666 child years of surveillance in last one year - 408 ALRI, 793 AURI and 243 control naso-pharyngeal samples were taken. S. pneumoniae was found positive in 61 samples (ALRI-32;7.8\%, AURI-20;2.5\%, Control-9;3.7\%), Haemophilus influenzae positive in 21 samples (ALRI-13;3.2\%, AURI-7;0.9\%, Control -1;0.4\%) and Staphylococcus aureus positive in 6 samples (ALRI-5;1.2\%, AURI-1;0.1\%, Control-Nil). The incidence of S. pneumoniae, H. influenzae and S. aureus in children with ALRI was 12.0 (95\% Cl:8.3-16.7), $4.9(95 \% \mathrm{Cl}: 2.7-8.1)$ and $1.9(95 \% \mathrm{Cl}: 0.7-4.2)$ per 1000 child years respectively in the population.

Conclusion: S. pneumoniae was found to be the most common bacterial respiratory pathogen followed by $H$. influenzae and S. aureus in children with ALRI in this community.

No conflict of interest 
ISPPD-0043

Pneumococcal Colonization and Carriage

\section{TRENDS IN ASYMPTOMATIC NASOPHARYNGEAL COLONIZATION WITH STREPTOCOCCUS PNEUMONIAE AFTER INTRODUCTION OF THE 13-VALENT PNEUMOCOCCAL CONJUGATE VACCINE IN CALGARY, CANADA}

L.J. Ricketson ${ }^{1}$, M.L. Wood ${ }^{2}$, O.G. Vanderkooi ${ }^{1}$, J.C. MacDonald ${ }^{3}$, J.D. Kellner ${ }^{1}$

${ }^{1}$ Pediatrics, University of Calgary, Calgary, Canada; ${ }^{2}$ Faculty of Medicine, University of Alberta, Edmonton, Canada; ${ }^{3}$ Population and Public Health, Alberta Health Services, Calgary, Canada

Background: We previously reported serotype-specific trends in pneumococcal nasopharyngeal (NP) colonization soon after introduction of the 7-valent pneumococcal conjugate vaccine (PCV7) in mid-2002. Our current aim is to describe later trends after PCV7 and early trends after PCV13 vaccine introduction in 2010.

Methods: The Calgary Area Streptococcus pneumoniae Epidemiology Research (CASPER) team conducted 10 pointprevalence surveys of pneumococcal nasopharyngeal colonization in healthy children aged 12 and 18 months and 4.5 years bi-annually from 2003-2005 (previously reported) and annually in 2006, 2010, 2011, and 2012.

Results:For surveys conducted in $2010-2012$, the proportion colonized was $13.2 \%$ compared with $19.9 \%$ in surveys conducted in 2003-2006 ( $p<0.001)$. Vaccination with 2 or more doses of PCV7 or PCV13, older age, and recent antibiotic use reduced the odds of colonization with any pneumococcus. By $2012,94 \%$ of all isolates were nonvaccine serotypes with $11 \mathrm{~A}, 15 \mathrm{~A} / \mathrm{B} / \mathrm{C}, 22 \mathrm{~F}, 23 \mathrm{~A} / \mathrm{B}, 35 \mathrm{~B} / \mathrm{F}$ representing $75 \%$ of all isolates.

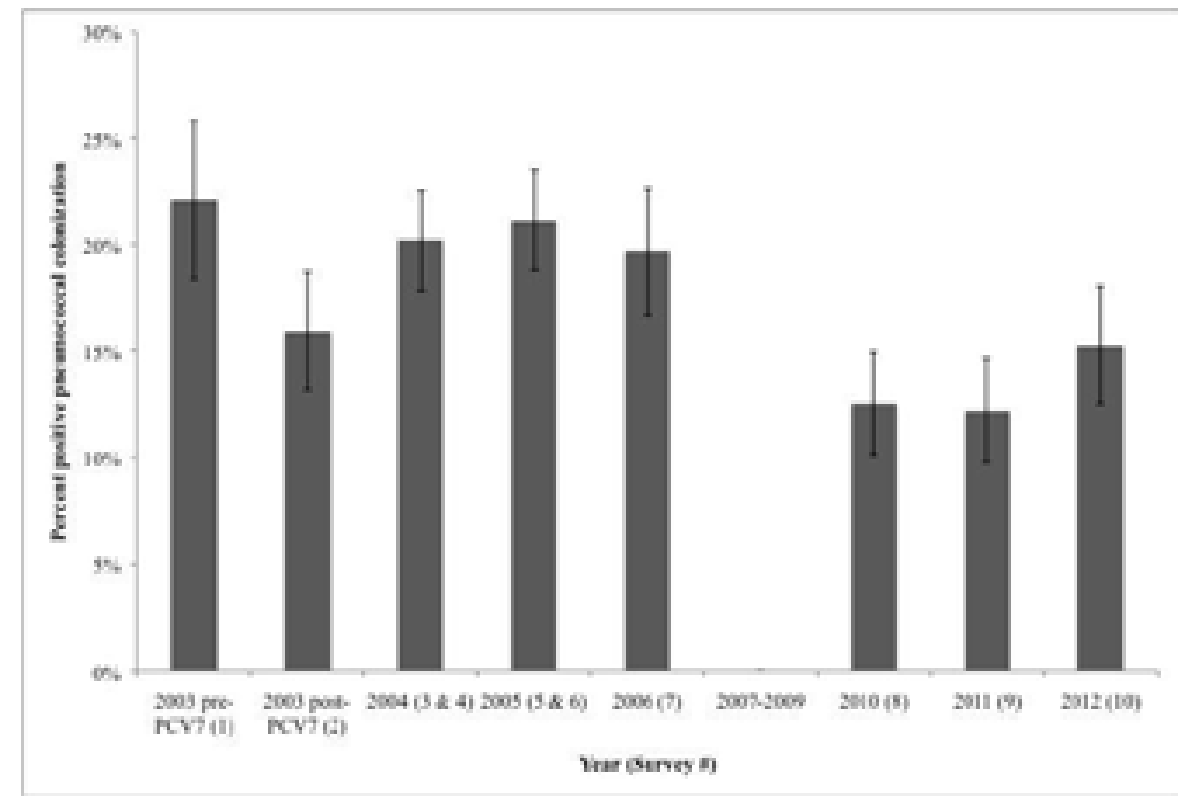

Conclusion: Pneumococcal nasopharyngeal colonization has changed profoundly since the introduction of conjugate vaccines and overall colonization by pneumococcus has declined in recent years. By 2012, non-vaccine serotypes have nearly completely replaced vaccine serotypes. The impact on clinical disease remains to be seen.

Conflict of interest

ISPPD-0125

Pneumococcal Colonization and Carriage

\section{PNEUMOCOCCAL COLONIZATION AS A SURROGATE ENDPOINT TO ASSESS VACCINE COVERAGE AND EFFICACY; A CROSS-SECTIONAL STUDY IN WARAO AMERINDIANS}

I.A. Rivera-Olivero ${ }^{1}$, B. del Nogal ${ }^{2}$, L.M. Verhagen ${ }^{3}$, M.C. Sisco ${ }^{1}$, K.M. Gómez ${ }^{1}$, R. Ramirez ${ }^{1}$, D. Bogaert ${ }^{4}$, P.W.M. Hermans $^{3}$, J.H. de Waard ${ }^{1}$

${ }^{1}$ Laboratorio de Tuberculosis, Instituto de Biomedicina, Caracas, Venezuela; ${ }^{2}$ Catedra de Pediatria, Hospital de Niños “JM de los Ríos”, Caracas, Venezuela; ${ }^{3}$ Division of Pediatric Infectious Diseases Department of Pediatrics, Radboud University Nijmegen Medical Center, Nijmegen, Netherlands; ${ }^{4}$ Department of Paediatric Infectious Diseases and Immunology, University Medical Center Utrecht-Wilhelmina Children's Hospital, Utrecht, Netherlands

Background and Aims: Nasopharyngeal colonization with Streptococcus pneumoniae is a surrogate marker for assessment of pneumococcal conjugate vaccine (PCV) coverage and efficacy. Here we investigate the usefulness of this endpoint for Warao people, an Amerindian population from Venezuela, living in the Orinoco River Delta. Methods: Surveillance studies of pneumococcal nasopharyngeal carriage were conducted in the years 2004, 2008 and 2012 among a total of 1313 Warao Amerindians children $\leq 9$ years old from 27 communities. PCVs have not yet been introduced in this population. Standard microbiological methods were used and pneumococci were serotyped by multiplex PCR and Quellung reaction.

Results: 827 pneumococcal isolates were collected over these 3 periods; an overall pneumococcal carriage rate of $63 \%$. Prevalence rates varied from 51\% in 2004 (188/370 samples) to $66 \%$ in 2008 (145/220 samples) and 68\% in 
2012 (494/723 samples). Also the prevalence of individual serotypes/groups covered by PCV13 varied considerably on the 3 time points with a natural increase in prevalence of serotype 1 ( 0 to $13 \%), 4$ (0 to $2 \%$ ) and 14 (6 to $12 \%$ ) and a natural decrease of serotype 18C (1 to 0.3\%). Serotypes/groups 6 and 23F remained stable (approx. 30\%) whereas non-vaccine serotypes 16, 34 and 38 emerged. The theoretical PCV13 coverage varied in time between $66 \%$ and $87 \%$.

Conclusions: Overall pneumococcal carriage rates, serotypes distribution and theoretical vaccine coverage were highly variable between 2004 and 2012. We conclude that surveillance of pneumococcal carriage at a single timepoint can under- or overestimate the long-term impact of PCV-13 in high-risk populations.

No conflict of interest

ISPPD-0515

Pneumococcal Colonization and Carriage

\section{EVOLUTION OF SEROTYPES CARRIED BY YOUNG CHILDREN IN PORTUGAL, A COUNTRY WITH WIDESPREAD USE OF PNEUMOCOCCAL CONJUGATE VACCINES (1996-2012)}

S. Nunes ${ }^{1}$, S. Félix ${ }^{1}$, C. Valente ${ }^{1}$, A.C. Paulo ${ }^{1}$, D.A. Tavares ${ }^{1}$, A.S. Simões ${ }^{1}$, S.T. Almeida ${ }^{1}$, A. Brito-Avô ${ }^{2}$, H. de Lencastre ${ }^{3}$, R. Sá-Leão ${ }^{1}$

${ }^{1}$ Laboratory of Molecular Microbiology of Human Pathogens, Instituto de Tecnologia Química e Biológica António Xavier, Oeiras, Portugal; ${ }^{2}$ Pediatrics, Private Pediatric Clinic, Lisbon, Portugal; ${ }^{3}$ Laboratory of Molecular Genetics, Instituto de Tecnologia Química e Biológica António Xavier, Oeiras, Portugal

Background and Aims: Portugal is one of the few countries where PCVs are widely used despite not being part of the national immunization program (NIP) nor being reimbursed. We evaluated the evolution of serotypes carried by young children over a 17-year period, spanning the pre-vaccine era up to the early-PCV13 era.

Methods: Nasopharyngeal swabs were obtained from children (0-6 years) attending day-care in Lisbon/Oeiras area in yearly cross-sectional studies conducted between 1996-2012. Pneumococci were isolated and serotyped. PCV use was extracted from the child's immunization bulletin.

Results: 8,313 swabs were obtained (average 593/year). Carriage remained stable (average 61.5\%). Use of PCV7 gradually increased to $>70 \%$ between $2007-2010$. PCV10 use was low ( $\leq 5 \%$ ). PCV13 use was $63 \%$ by 2012 (among those born after 2009). Major shifts have occurred in circulating serotypes (Figure). PCV7 types have declined substantially but are still in circulation. In 2012, the 6-extra PCV13 types and non-PCV13 types (including 6C) accounted for $\sim 10 \%$ and $\sim 75 \%$ of all isolates, respectively. In 2012 , the 6-extra PCV13 types were significantly less frequent among PCV13 vaccinated than among PCV13 non-vaccinated carriers $(2.9 \%$ vs $19.6 \%, p<0.001)$.

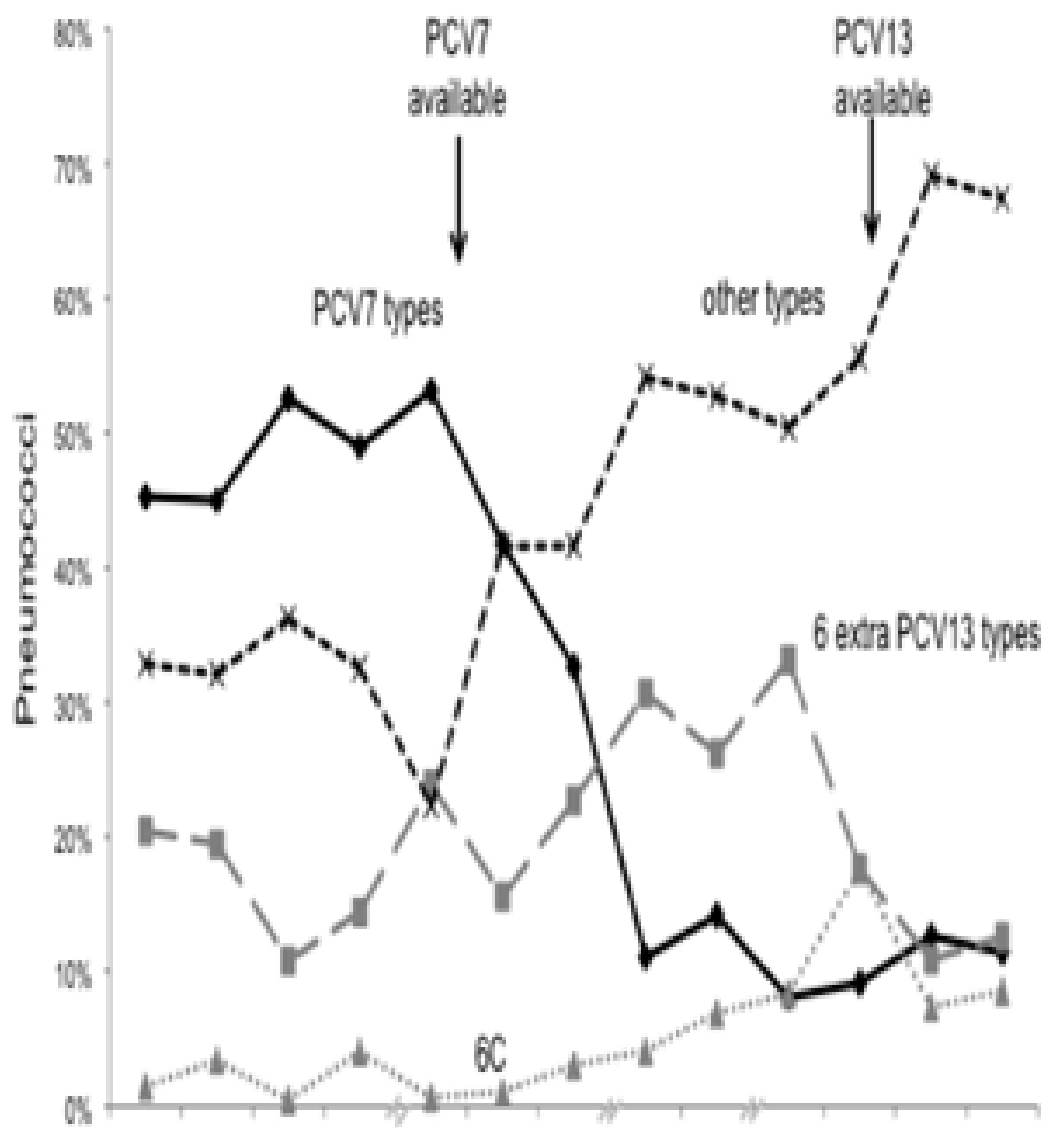


Conclusion: Significant changes in carried serotypes have been occurring although not as dramatic as those reported in countries that introduced PCVs in the NIP. Whether these changes will be sustained is uncertain. Health authorities should consider universal introduction of PCVs in Portugal.

Conflict of interest

ISPPD-0039

Pneumococcal Colonization and Carriage

\title{
SEROTYPE DISTRIBUTION AND ANTIBIOTICS SUSCEPTIBILITY OF STREPTOCOCCUS PNEUMONIAE CARRIAGE IN CHILDREN INFECTED WITH HUMAN IMMUNODEFICIENCY VIRUS IN JAKARTA, INDONESIA
}

\author{
D. Safari ${ }^{1}$, N. Kurniati ${ }^{2}$, L. Waslia ${ }^{3}$, M. Khoeri ${ }^{1}$, S. Mudaliana ${ }^{1}$, T. Putri ${ }^{4}$, D. Subekti ${ }^{3}$, D. Bogaert ${ }^{5}$, K. Trizinski ${ }^{5}$ \\ ${ }^{1}$ Microbiology, Eijkman Institute for Molecular Biology, Jakarta, Indonesia; ${ }^{2}$ Child Health, Faculty of Medicine University of Indonesia/Cipto Man- \\ gunkusumo Hospital, Jakarta, Indonesia; ${ }^{3}$ Microbiology, Eijkman Oxford Clinical Research Unit, Jakarta, Indonesia; ${ }^{4}$ Biology, Gajah Mada Univer- \\ sity, Yogyakarta, Indonesia; ${ }^{5}$ Pediatrics, University medical Center Utrecht, Utrecht, Netherlands
}

Objective: To provide epidemiological data on Streptococcus pneumoniae carriage in high risk population, this study focuses on the serotype distribution and antibiotics susceptibility of S. pneumoniae carriage in HIV-infected children in Jakarta, Indonesia.

Materials and Findings: Nasopharyngeal swabs were collected from 90 HIV sero-positive children age 4 to 144 months. S. pneumoniae was identified by conventional and molecular methods. Serotyping, antibiotics susceptibility test, and sequence type were performed by sequential multiplex PCR, the disk diffusion method and MLST respectively. We identified $S$. pneumoniae in 42 children (46.7\%). Serotype $19 \mathrm{~F}$ was identified most commonly ( $n$ $=8,19.0 \%$ ) followed by $9 \mathrm{~A}$ and $6 \mathrm{~A} / \mathrm{B}(n=4,8.5 \%), 23 \mathrm{~F}$ ( 3 isolates), $9 \mathrm{~V}, 35 \mathrm{~B}, 11 \mathrm{~A}$ (two isolates each) and serotypes $18,12 \mathrm{~F}, 15 \mathrm{~B} / \mathrm{C}, 3$ and $35 \mathrm{~F}$ (single isolates each). Antibiotic susceptibility was observed for chloramphenicol (85.4\%), erythromycin (73.2\%), clindamycin (78.0\%)) and sulphamethoxazole-trimethoprim (39.0\%). Furthermore, multidrugs resistant isolates of serotype $19 \mathrm{~F}$ belonging to the ST320 clone ( 3 isolates) and the ST263 clone ( 2 isolates) were identified.

Conclusion: Our study gives insight into prevalence and serotype distribution of S. pneumoniae strains among young HIV patients in Indonesla. These findings may help to decide upon potential preventive strategies targeting invasive pneumococcal disease in Indonesia.

No conflict of interest

ISPPD-0375

Pneumococcal Colonization and Carriage

\section{STREPTOCOCCUS PNEUMONIAE SEROTYPE PROFILE IN NASOPHARYNGEAL ISOLATES OF HEALTHY CHILDREN IN SOUTH INDIA}

\author{
J. Sankar ${ }^{1}$ \\ ${ }^{1}$ Pediatrics, Kanch Kamakoti Childs Trust Hospital, Chennai, India
}

Background: Invasive disease due to Streptococcus pneumonia is one of the commonest causes of death in children. Some pneumococcal serotypes that colonizes nasopharynx of healthy children can lead to invasive disease. It is important to identify the serotypes colonizing the nasopharynx to enable prevention by way of vaccination.

Aims: To determine the serotype profile of $S$. pneumonia isolated from healthy children.

Methods: A total of 500 children between 6 months to 5 years were included. A detailed questionnaire was filled to delineate the vaccination status, underlying risk factors and socioeconomic status. Nasopharyngeal swabs were taken as per protocol and plated immediately.. Serotyping was done using Statens kit.

Results: 45/500 grew S. pneumoniae. 6B was the commonest serotype identified (16/45), most of them were infants with overcrowding and improper ventilation. 6 children who had $23 \mathrm{~F}$ serotype were $>2$ years of age and overcrowding was again found to be a significant risk factor. $19 \mathrm{~F}$ was seen in 5 children and there was no significant risk factor associated. Serotypes 14, 4,18C, $19 \mathrm{~V}$ and nontypeable were seen in 3, 3, 1, 1, and 10 children respectively. Factors like duration of breast feeding, smoking and previous respiratory infection did not have any influence on the carriage rate.

Conclusion: Nasopharyngeal carriage was found to be common in healthy children without any significant risk factors. Serotype 6B was the commonest serotype isolated.

No conflict of interest 


\section{COMPARISON OF THE 2003 AND 2013 WORLD HEALTH ORGANIZATION (WHO) GUIDELINES FOR DETECTING UPPER RESPIRATORY CARRIAGE OF STREPTOCOCCUS PNEUMONIAE}

C. Satzke ${ }^{1}$, P. Turner ${ }^{2}$, A. Virolainen-Julkunen ${ }^{3}$, P.V. Adrian ${ }^{4}$, M. Antonio ${ }^{5}$, K.M. Hare ${ }^{6}$, A.M. Henao-Restrepo ${ }^{7}$, A.J. Leach ${ }^{6}$, K.P. Klugman ${ }^{8}$, B.D. Porter ${ }^{1}$, R. Sá-Leão ${ }^{9}$, J.A. Scott ${ }^{10}$, H. Nohynek ${ }^{11}$, K.L. O'Brien ${ }^{12}$

${ }^{1}$ Pneumococcal Research, Murdoch Childrens Research Institute, Parkville, Australia; ${ }^{2}$ Cambodia-Oxford Medical Research Unit, Angkor Hospital for Children, Siem Reap, Cambodia; ${ }^{3}$ Department of Infectious Disease Surveillance and Control, National Institute for Health and Welfare, Helsinki, Finland; ${ }^{4} \mathrm{MRC} /$ Wits Respiratory and Meningeal Pathogens Research Unit, University of the Witwatersrand, Johannesburg, South Africa; ${ }^{5}$ Vaccinology Theme, Medical Research Council Unit, Banjul, Gambia; ${ }^{6}$ Ear Health Research Program, Menzies School of Health Research, Darwin, Australia; ${ }^{7}$ Initiative for Vaccine Research, World Health Organization, Geneva, Switzerland; ${ }^{8}$ Rollins School of Public Health, Emory University, Atlanta, USA; ${ }^{9}$ Laboratory of Molecular Microbiology of Human Pathogens, Instituto de Tecnologia Química e Biológica, Oeiras, Portugal; ${ }^{10}$ Epidemiology and Demography, KEMRI-Wellcome Trust Research Programme, Kilifi, Kenya; ${ }^{11}$ Vaccine Programme Unit, National Institute for Health and Welfare, Helsinki, Finland; ${ }^{12}$ Department of International Health, Johns Hopkins Bloomberg School of Public Health, Baltimore, USA

Background and Aims: We recently published a set of updated World Health Organization (WHO) standard methods for detecting pneumococcal carriage, including sample collection, transport and storage, pneumococcal identification and serotyping.

Methods: We identified key changes in the 2013 revised recommendations compared with the 2003 guidelines.

Results: A single nasopharyngeal swab in STGG medium remains the sample of choice. Important changes are:

\begin{tabular}{|c|c|c|}
\hline & Minor changes & New recommendations or major changes \\
\hline Specimen type & Document swab quality & $\begin{array}{l}\text { Collect nasopharyngeal and oropharyngeal swabs } \\
\text { from adults. }\end{array}$ \\
\hline Swab material & $\begin{array}{l}\text { Calcium alginate, rayon, Dacron or } \\
\text { nylon swabs are acceptable for cul- } \\
\text { ture-based studies. }\end{array}$ & $\begin{array}{l}\text { Swabs of inert material (e.g. nylon or Dacron) } \\
\text { should be used for molecular methods. } \\
\text { Flocked swabs have many desirable characteristics. }\end{array}$ \\
\hline $\begin{array}{l}\text { Swab collection, } \\
\text { transport and storage }\end{array}$ & $\begin{array}{l}1 \mathrm{ml} \mathrm{STGG} \text { medium. } \\
\text { Freeze at }-80^{\circ} \mathrm{C} \text { as soon as possible, } \\
\text { no 'safe limit' for } 4^{\circ} \mathrm{C} \text { storage, store } \\
\text { at }-20^{\circ} \mathrm{C} \text { for days (not weeks). }\end{array}$ & \\
\hline Culture & $\begin{array}{l}10 \mu \text { of specimen, record if larger } \\
\text { volume. } \\
\text { COBA and CNA plates acceptable al- } \\
\text { ternatives to gentamicin-blood agar. } \\
\text { Recording semi-quantitative growth } \\
\text { is optional }\end{array}$ & \\
\hline Selecting colonies & & $\begin{array}{l}\text { At least one } \alpha \text {-haemolytic colony (representing } \\
\text { the dominant morphology). If other colonies are } \\
\text { tested, results should be analysed separately. }\end{array}$ \\
\hline Identification & $\begin{array}{l}\text { Real-time lytA is the current best } \\
\text { molecular identification approach. }\end{array}$ & $\begin{array}{l}\text { Optochin non-susceptible isolates should be tested } \\
\text { for bile solubility. }\end{array}$ \\
\hline Serotyping & $\begin{array}{l}\text { 'Wet' or 'dry' Quellung method is } \\
\text { gold-standard. } \\
\text { Latex agglutination acceptable, other } \\
\text { methods may be suitable. }\end{array}$ & \\
\hline
\end{tabular}

Important areas for future research will be described.

Conclusion: There are important differences between the 2003 and 2013 guidelines. Adherence to standard methods will reduce variability when conducting pneumococcal carriage studies.

Conflict of interest 


\title{
CHANGES IN NASOPHARYNGEAL PNEUMOCOCCAL LOAD WITH SYMPTOMATIC VIRAL INFECTION IN KENYAN
} CHILDREN

\author{
S.C. Morpeth ${ }^{1}$, P. Munywoki ${ }^{1}$, L.L. Hammitt ${ }^{2}$, A. Bett ${ }^{1}$, D. Koech ${ }^{1}$, C. Onyango ${ }^{1}$, D.R. Murdoch ${ }^{3}$, D.J. Nokes ${ }^{4}$, \\ J.A.G. Scott ${ }^{5}$ \\ ${ }^{1}$ Epidemiology and Demography, KEMRI-Wellcome Trust Research Programme, Kilifi, Kenya; ${ }^{2}$ Department of International Health, Johns Hop- \\ kins Bloomberg School of Public Health, Baltimore, USA; ${ }^{3}$ Department of Pathology, University of Otago, Christchurch, New Zealand; \\ ${ }^{4}$ School of Life Sciences and WIDER, University of Warwick, Coventry, United Kingdom; ${ }^{5}$ Department of Infectious Diseases Epidemiology, Lon- \\ don School of Hygiene and Tropical Medicine, London, United Kingdom
}

Background and Aims: The impact of viral upper respiratory tract infection (URTI) on pneumococcal load in the human nasopharynx is uncertain. We hypothesised that acquisition of a viral URTI would be associated with an increase in nasopharyngeal pneumococcal concentration in children $<5$ years, followed by a subsequent decrease. Methods: All members of 47 households from Kilifi District, coastal Kenya, had nasopharyngeal swabs (NPS) collected twice weekly during Jan-June 2010 and tested by a respiratory multiplex PCR. Swabs from children $<5$ years old collected two weeks before, during and four weeks after a first episode of Respiratory Syncytial Virus (RSV) or rhinovirus with URTI symptoms were also tested by quantitative real-time PCR for lytA and alu targets as a measure of pneumococcal load per $\mu \mathrm{g}$ of human DNA present in the swab transport medium.

Results: Fifty-three viral episodes were available for study. Pneumococcal carriage was universal among study participants and co-infecting viruses were present in $35 \%$ of swabs. The geometric mean increase in pneumococcal concentration with acquisition of viral infection was 4-fold, $p<0.001$, and remained so when the analysis was restricted to swabs without co-infecting viruses. Pneumococcal concentration fell 0.6-fold after viral URTI but without statistical significance, $p=0.144$.

Conclusion: The modest increase in pneumococcal concentration may be an important contributing factor to the development of bacterial pneumonia or invasive pneumococcal disease among children with preceding viral respiratory tract infections at a population level. The impact on population level transmission of pneumococcal carriage during viral URTI is potentially even greater.

No conflict of interest

ISPPD-0202

Pneumococcal Colonization and Carriage

\section{STREPTOCOCCUS PNEUMONIAE CARRIAGE SEROTYPES AMONG CHILDREN AGED 3 MONTHS TO 5 YEARS IN LOWER SINDH, PAKISTAN - DETERMINATION BY REAL-TIME PCR}

\author{
S. Shakoor ${ }^{1}$, S. Muneer ${ }^{1}$, V. Srinivasan ${ }^{2}$, F. Jehan ${ }^{1}$, I. Nisar ${ }^{1}$, A. Hotwani ${ }^{1}$, S. Qureshi ${ }^{1}$, B. Beall ${ }^{2}$, C. Whitney ${ }^{3}$, A. Zaidi $^{1}$ \\ ${ }^{1}$ Pediatrics and Child Health, Aga Khan University, Karachi, Pakistan; ${ }^{2}$ Streptococcus laboratory Respiratory Disease Branch, Centers for Dis- \\ ease Control, Atlanta, USA; ${ }^{3}$ Respiratory Disease Branch, Centers for Disease Control, Atlanta, USA
}

Background: Nasopharyngeal colonization plays a key role in pathogenesis and spread of pneumococcal disease. With the worldwide introduction of pneumococcal conjugate vaccine (PCV), it is imperative that carriage serotypes in children are determined in areas of vaccine introduction. PCV10 was introduced in Pakistan in April 2013.

Methods: From two districts of Sindh (Karachi and Matiari) 698 nasopharyngeal swabs were collected from children aged 3 months to 5 years in January 2013 (before PCV-10 introduction). Swabs were subcultured on sheep blood agar and isolates with suggestive colony morphology were tested for optochin sensitivity. We found that 561 $(80.4 \%)$ of the specimens yielded pneumococci. A sampling of 100 isolates, representing 100 different swabs, were shipped to the Centers for Disease Control and prevention(CDC), Atlanta GA, for serotyping using sequential real time triplexed-PCR reactions according to published CDC protocols.

Results: Fifty-three of 100 isolates have been serotyped. Of these $34 \%(n=18)$ were $6 A / B / C / D, 20.8 \%(n=11)$ were $23 F, 15 . \% 1(n=8)$ were $19 A, 7.5 \%(n=4)$ were $19 F, 7.5 \%(n=4)$ were $18 C / F / B / A, 7.5 \%(n=4)$ were $9 V / A, 5.7 \%(n=3)$ were 14 and $1.9 \%(n=1)$ were determined to be serotype 4 . Serotype determination of remaining 47 isolates is ongoing. Resolution of common serogroups into individual serotypes will be carried out by Quellung at CDC.

Conclusion: This is the first report of nasopharyngeal pneumococcal serotypes from Pakistan. Nasopharyngeal carriage rate among children in lower Sindh is high. Data obtained so far reveals high prevalence of vaccine serotypes (all either PCV10 or PCV10-related). Complete serotyping of all 561 pneumococcal isolates is critical to confirm these early observations.

No conflict of interest 
ISPPD-0493

Pneumococcal Colonization and Carriage

\title{
DISPARATE RATES OF STREPTOCOCCUS PNEUMONIAE COLONIZATION IN CHILDREN < 5 YEARS WITH A HISTORY OF WHEEZING
}

\author{
K.M. Shea ${ }^{1}$, A. Rabbani ${ }^{1}$, S.I. Pelton ${ }^{2}$ \\ ${ }^{1}$ Epidemiology, Boston University School of Public Health, Boston, USA; ${ }^{2}$ Pediatrics, Boston University School of Medicine, Boston, USA
}

Background: Children with certain chronic diseases are at increased risk for IPD, but it is not known whether this is because they are more likely to be colonized with Streptococcus pneumoniae, or because they are more likely to develop disease once colonization has occurred.

Methods: We compared the rate of $S$. pneumoniae colonization in children $<5$ years with underlying comorbidities to the rate in children without comorbidities. Demographic information and nasopharyngeal specimens were collected from 2,441 children $<5$ years seen in primary care at a large urban U.S. hospital after introduction of PCV13, from July 2010-July 2013. Standard microbiologic methods were used to characterize pneumococcal serotypes, and patient records were used to ascertain comorbidities.

Results: One third ( $n=937$ ) of our study population had $\geq 1$ comorbidity. Among 2,211 recorded comorbidities, wheezing was the most common (61.6\%), followed by sickle cell haemoglobinopathies (12.3\%), prematurity $(12.2 \%)$, and chronic heart disease (7.0\%). Children with a diagnosis of wheeze or asthma in their clinical record had a higher rate of colonization than children without a wheeze or asthma diagnosis ( $26 \% \mathrm{vs} .21 \%, \mathrm{p}=0.054)$. Although not statistically significant, children with other chronic lung ( $22 \%$ vs. $18 \%)$ and kidney diseases $(30 \%$ vs. $22 \%)$ also had higher rates of $S$. pneumoniae colonization.

Conclusion: Children with medical record evidence of wheeze appear to have increased rates of S. pneumoniae colonization. Future analyses will provide insight into whether this association persists after controlling for covariates such as age and vaccination.

Funding: ASPIRE Award in Pediatric Vaccine Research from Pfizer, Inc.

Conflict of interest

ISPPD-0432

Pneumococcal Colonization and Carriage

\section{NASOPHARYNGEAL CARRIAGE OF STREPTOCOCCUS PNEUMONIAE AMONG CHILDREN <5 YEARS OF AGE BEFORE THE INTRODUCTION OF PNEUMOCOCCAL CONJUGATE VACCINE IN MOZAMBIQUE}

\author{
B. Sigaúque ${ }^{1}$, S. Massora ${ }^{2}$, S. Acácio ${ }^{3}$, R. Santos ${ }^{4}$, D. Vubil ${ }^{5}$, F. Pimenta ${ }^{6}$, I. Moura ${ }^{6}$, C. Whitney ${ }^{6}$, E.M. Macete ${ }^{7}$, \\ P.L. Alonso ${ }^{7}$, M.G. Carvalho 6 , J.R. Verani ${ }^{6}$ \\ ${ }^{1}$ Clinic, Centro de Investigação em Saúde de Manhiça, Maputo, Mozambique; ${ }^{2}$ Clinic, Centro de Investogação em Saúde de Manhiça, Maputo, \\ Mozambique; ${ }^{3}$ clinic, Centro de Investogação em Saúde de Manhiça, Maputo, Mozambique; ${ }^{4}$ Hospital Central de Nampula, Ministério de Saúde, \\ Nampula, Mozambique; ${ }^{5}$ clinic, Centro de Investigação em Saúde de Manhiça, Maputo, Mozambique; ${ }^{6}$ Respiratory Diseases Branch, Cent- \\ ers for Disease Control and Prevention, Atlanta, USA; ${ }^{7}$ clinic, Centro de Investogaçao em Saude de Manhiça, Maputo, Mozambique
}

Background: Nasopharyngeal carriage is a precursor for pneumococcal disease and can be useful for evaluating pneumococcal conjugate vaccine (PCV) impact. We studied pre-PCV pneumococcal carriage among HIV-infected and -uninfected children in Mozambique.

Methods: Between October 2012 and March 2013, we enrolled HIV-infected children age $<5$ years presenting for routine care at seven HIV clinics in 3 sites, including Maputo (urban-south), Nampula (urban-north), and Manhiça (rural-south). We also enrolled a random sample of HIV-uninfected children $<5$ years old from a demographic surveillance site in Manhiça. A single nasopharyngeal swab was obtained and cultured following broth enrichment. Pneumococcal isolates were serotyped by Quellung reaction.

Results: Among 312 HIV-uninfected children enrolled from the community, 247 (79\%) carried pneumococcus. Among 415 children enrolled from HIV clinics, the prevalence was $81 \%$, with no significant difference by site (Maputo 92/123 [75\%], Nampula 82/98 [84\%], Manhiça 164/194 [85\%], p=0.086). Among 601 pneumococcal isolates from 585 children, serotypes $19 \mathrm{~F}(13 \%), 23 \mathrm{~F}(13 \%), 6 \mathrm{~A}(9 \%), 6 \mathrm{~B}(6 \%), 19 \mathrm{~A}(5 \%), 13(5 \%)$ and $14(5 \%)$ were most common; serotype could not be determined for $36(6 \%)$. The proportion of isolates included in the 10- and 13 -valent vaccines (PCV10 and PCV13) was $47 \%$ and $64 \%$, respectively, with no significant differences by HIV status. Conclusion: Pneumococcal carriage was common, with little variation by geographic region or HIV status. Serotype coverage of PCV10, which was introduced in Mozambique in April 2013, was lower than that of PCV13. Ongoing carriage studies will show whether PCV10 will have similar benefits for HIV-infected and -uninfected children.

No conflict of interest 
ISPPD-0172

Pneumococcal Colonization and Carriage

\title{
THE IMPACT OF MULTIPLE SEROTYPE CARRIAGE DETECTION BY MICROARRAY-BASED MOLECULAR SEROTYPING IN TWO UK NASOPHARYNGEAL CARRIAGE STUDIES
}

\author{
M. Slack ${ }^{1}$, J. Hinds' ${ }^{2}$, K. Gould ${ }^{2}$, S. Manglani ${ }^{1}$, Y.H. Choi ${ }^{3}$, C.L. Sheppard ${ }^{1}$, E. Miller ${ }^{4}$ \\ ${ }^{1}$ Respiratory \& Vaccine Preventable Bacteria Reference Unit, Public Health England, London, United Kingdom; ${ }^{2}$ Bacterial Microar- \\ ray Group at St George's, St George's University of London, London, United Kingdom; ${ }^{3}$ Immunisation Clinical Evaluation Hepatitis and Transfu- \\ sion, Public Health England, London, United Kingdom; ${ }^{4}$ Immunisation Hepatitis and Blood Safety Department, Public Health England, London, \\ United Kingdom
}

Background and Aims: Determining nasopharyngeal carriage of pneumococcal serotypes is a valuable epidemiological surveillance tool. However, current serotyping methodologies are limited in their ability to detect multiple serotype carriage, thus underestimating the complexity of carriage by missing rare or low abundant serotypes. The Health Protection Agency conducted a longitudinal nasopharyngeal carriage study (A) of 489 individuals in England over 10 months pre-PCV7 in 2001/2 and a cross-sectional study (B) of 400 individuals post-PCV7in 2009. Conventional culture and serotyping identified 932 and 125 swabs containing pneumococci respectively. The swabs were stored at $-70^{\circ} \mathrm{C}$ in skimmed milk, tryptone, glucose, glycerol broth (STGG). The present study used a DNA microarray to re-examine a sample of swabs previously yielding a single serotype to assess the prevalence of co-colonisation with multiple serotypes.

Methods: 410/932 pneumococcal-positive swabs from study A and 115/125 from study B were re-examined using a molecular serotyping DNA microarray (Hinds et al).

Results: $17 \%$ samples contained multiple serotypes: $14 \%$ contained two, $2 \%$ three and $0.4 \%$ four serotypes respectively. A further $13 \%$ samples contained "serotype variants" where cps loci did not match known serotypes; possibly indicating non-typable pneumococci, novel serotypes or closely related Streptococcus spp.

Conclusion: The DNA microarray detected $17 \%$ co-colonisation in a subset of swabs from previous carriage studies, despite long-term storage at $-70^{\circ} \mathrm{C}$ for several years. Conventional methods had only detected $0.16 \%$ co-colonisation. Emergence of non-vaccine serotypes post-conjugate vaccine introduction may partly result from unmasking rather than serotype replacement. Co-colonisation will have a significant impact on future modeling studies.

No conflict of interest

ISPPD-0458

Pneumococcal Colonization and Carriage

\section{RECOMBINANT PNEUMOLYSIN AND PNEUMOLYSIN PRODUCING STREPTOCOCCUS PNEUMONIAE HAVE DIFFERENT EFFECTS ON TLR4 IN HUMAN MONOCYTE DERIVED DENDRITIC CELLS}

L. Spelmink ${ }^{1}$, L. Plant ${ }^{1}$, B. Henriques-Normark ${ }^{1}$

${ }^{1}$ Dept. Microbiology Tumor and Cell Biology (MTC), Karolinska Institutet, Stockholm, Sweden

Background: The cholesterol-dependent pore-forming toxin pneumolysin is a key virulence factor of Streptococcus pneumoniae. It has been shown to be the cause of apoptosis and complement activation, as well as cause damage to tissues in the lung and brain. Detection of pneumolysin by TLR4 has been described; however reports are conflicting, depending on the model and bacterial strain used. There are very few reports about the role of TLR4 in the detection of pneumolysin in human primary immune cells.

Methods: Human monocyte-derived dendritic cells (DCs) were treated with siRNA to TLR4 and infected with unencapsulated serotype 4 pneumococci (T4R), the pneumolysin mutant (T4R $\Delta$ ply) or treated with highly purified recombinant pneumolysin. The secretion of the pro-inflammatory cytokine IL-12p70 was measured in the cell culture supernatant of infected DCs.

Results: The pneumolysin mutant T4R $\Delta$ ply induces IL-12p70 secretion levels in DCs which are up to 2000 times higher than the levels induced by T4R. The IL-12p70 secretion in response to both strains is dependent on the uptake of the bacteria by DCs and does not require TLR4. Recombinant pneumolysin, in contrast, induces TLR4dependent secretion of IL-12p70.

Conclusion: We do not currently understand the complex signaling effects of pneumolysin on DCs. Our results indicate that the stimulation with purified toxin has different effects on DCs than in the context of infection with whole bacteria. We hypothesize that pneumolysin has an inhibitory function in the context of bacterial infections and we are setting out on investigating this effect with our future studies.

No conflict of interest 


\title{
VACCINE-INDUCED CHANGES IN NASOPHARYNGEAL PNEUMOCOCCAL CARRIAGE AFTER REPLACING THE 7-VALENT PNEUMOCOCCAL CONJUGATE VACCINE WITH THE 13-VALENT VACCINE IN NORWAY
}

\author{
A. Steens ${ }^{1}$, I.S. Aaberge ${ }^{1}$, D.A. Caugant ${ }^{1}$, D.F. Vestrheim ${ }^{2}$ \\ ${ }^{1}$ Division of Infectious Disease Control, Norwegian Institute of Public Health, Oslo, Norway; ${ }^{2}$ Division of Infectious Disease Control, Norwe- \\ gian Institute of Public Health + European Programme for Public Health Microbiology Training (EUPHEM) European Centre for Disease Preven- \\ tion and Control (ECDC) Sweden, Oslo, Norway
}

Introduction: Because children are the main transmitters of pneumococci, carriage studies focussing on the young provide good information on the circulating serotypes. In 2011, the 13-valent pneumococcal conjugate vaccine (PCV13) replaced the 7-valent vaccine (PCV7), which was used since 2006 for childhood immunisation in Norway. We assessed vaccine-induced changes in carriage among children younger than 6 years by comparing the PCV13 sample with a pre-vaccine and PCV7 sample.

Methods: A cross-sectional sample with questionnaire data and nasopharyngeal swabs was obtained in autumn 2013 from children attending day-care centres in and near Oslo, the Norwegian capital. All isolates were serotyped and antimicrobial-susceptibility was tested. Results were compared to samples obtained in autumn 2006 (611 swabs, 539 isolates) and autumn 2008 (602 swabs, 563 isolates).

Results: We obtained 367 swabs with 244 isolates (carriage-prevalence $62 \%$ [95\% Cl 54-70]) but continue sampling until $\geq 800$ swabs are obtained. Carriage was highest among the youngest ( $<24$ months; $80 \%[95 \% \mathrm{Cl} 67-89])$. In the pre-vaccine and PCV7 samples, the overall prevalence was $78 \%[95 \% \mathrm{Cl} 73-82]$ and $80 \%[95 \% \mathrm{Cl} 76-84]$. The prevalence of PCV13-serotypes decreased from 540/1000 to 316/1000 to 93/1000 children, respectively. The decrease was observed in all age-groups. While after PCV7 introduction the prevalence of non-vaccine serotypes increased from 336/1000 to 606/1000, after switching to PCV13, the prevalence remained constant (569/1000). Non-PCV serotypes 21, 35F, $11 \mathrm{~A}$ and $23 \mathrm{~A}$ have become the dominant serotypes.

Conclusion: Preliminary results indicate decreased carriage of vaccine serotypes after switching from PCV7 to PCV13, with no increase in carriage of non-vaccine serotypes.

No conflict of interest

ISPPD-0318

Pneumococcal Colonization and Carriage

\section{ASSOCIATIONS BETWEEN BACTERIAL PATHOGENS IN THE NASOPHARYNX OF HIV INFECTED CHILDREN AND MOTHERS}

\author{
B. Arya ${ }^{1}$, S. Das Bhattacharya ${ }^{1}$, S. Niyogi' ${ }^{2}$ S. Bhattacharyya ${ }^{3}$, S. Mandal ${ }^{2}$, R. Saurav Das ${ }^{1}$ \\ ${ }^{1}$ School of Medical Science and Technology, Indian Institute of Technology-Kharagpur, Kharagpur, India; ${ }^{2}$ Microbiology, National Institute of Chol- \\ era and Enteric Diseases, Kolkata, India; ${ }^{3}$ Pediatrics, Midnapore Medical College, Midnapore (currently at Medical College Kolkata), Kolkata, India
}

Background: Three leading pathogens responsible for bacteremic pneumonia in HIV infected children are Streptococcus pneumoniae, Staphylococcus aureus, and Haemophilus influenzae. Bacterial interference, or competition amongst organisms in the nasopharyngeal space, is well documented. Studies investigating dynamics of these associations in HIV infected mother and children are not well reported.

Aim: To investigate bacterial associations of S. pneumoniae, S. aureus, and H. influenzae in the nasopharynx of mother and children with HIV infection

Methods: Four nasopharyngeal samples were collected from pneumococcal vaccine naïve, HIV infected children ages 2-15 years, and their mothers, at two month intervals over the course of six months. Calcium alginate swabs were collected and placed in STGG media and processed in the microbiology laboratory using standardized protocols.

Results: We have collected about 404 from children and 375 from mother, till now. Overall dual carriage with Pneumococcus and S. aureus was found to be $13 / 404$ in children and $3 / 375$ in mothers (all HIV infected). Similarly, dual carriage with pneumococcus and H.influenzae was found to be $25 / 404$ in children and 2/375 in mothers. A positive association was seen between pneumococcus and $H$. influenzae [Odds ratio (for mother): $13.84 ; 95 \% \mathrm{Cl}$ : 1.06-126.82; Odds ratio (for children): $5.62 ; 95 \% \mathrm{Cl}: 2.74-11.66]$. Any association between Pneumococcus and $S$. aureus was not found in HIV infected mother or children [Odds ratio (for mother) : 1.29; $95 \% \mathrm{Cl}$ : 0.23-4.78; Odds ratio (for children) : $0.67 ; 95 \% \mathrm{Cl}: 0.32-1.33]$.

No conflict of interest 
ISPPD-0527

Pneumococcal Colonization and Carriage

\section{EVALUATION OF THE NEGATIVE PREDICTIVE VALUE OF PNEUMOCOCCAL CARRIAGE FOR ASSESSING PNEUMOCOCCAL PNEUMONIA IN THE PERCH STUDY}

The PERCH Study Group ${ }^{1}$

${ }^{1}$ International Vaccine Access Center, Johns Hopkins Bloomberg School of Public Health, Baltimore, USA

Background: Low specificity of definitions for pneumococcal pneumonia (PP) limits evaluation of pneumococcal conjugate vaccine (PCV) impact. We investigated the usefulness of absent Streptococcus pneumoniae carriage in assessing PP.

Methods: PERCH studies severe/very severe hospitalized pneumonia among children aged 28d-59m. The presence of nasopharyngeal (NP) carriage by PCR (lytA) or culture was calculated among cases using microbiologicallyconfirmed pneumococcal pneumonia (MCPP), WHO-defined alveolar consolidation (CXR-AC), and CXR-AC or other infiltrate plus elevated C-reactive protein (CRP) as outcome measures. Odds ratios, adjusted for site and age, were calculated.

Results:

\begin{tabular}{|l|l|l|l|l|l|l|}
\hline Outcome & $\begin{array}{l}\text { Carriage } \\
\text { in group } \\
\text { without } \\
\text { outcome } \\
(\%)\end{array}$ & $\begin{array}{l}\text { Carriage in } \\
\text { group with } \\
\text { outcome } \\
\text { (Sensitivity) } \\
(\%)\end{array}$ & $\begin{array}{l}\text { Specificity } \\
(\%)\end{array}$ & $\begin{array}{l}\text { Positive } \\
\text { Predictive } \\
\text { Value (\%) }\end{array}$ & $\begin{array}{l}\text { Negative } \\
\text { Predictive } \\
\text { Value (\%) }\end{array}$ & aOR (95\% CI) \\
\hline $\begin{array}{l}\text { MCPP (n=47) } \\
\text { vs. Non-MCPP } \\
\text { (n=2512) }\end{array}$ & 78.5 & 97.9 & 21.5 & 2.3 & 99.8 & $8.3(1.1-61.3)$ \\
\hline $\begin{array}{l}\text { CXR-AC (n=420) } \\
\text { vs. Other infiltrate/ } \\
\text { normal (n=1192) }\end{array}$ & 79.9 & 78.6 & 20.1 & 25.7 & 72.7 & $1.2(0.9-1.6)$ \\
\hline $\begin{array}{l}\text { CXR-AC or (other } \\
\text { infiltrate }+ \\
\text { CRP>=40 mg/l) } \\
\text { (n=165) vs. } \\
\text { Normal/CRP<40 } \\
\text { mg/l (n=207)* }\end{array}$ & 61.8 & 73.3 & 38.2 & 48.6 & 64.2 & $1.7(1.1-2.7)$ \\
\hline
\end{tabular}

*South Africa Only

NPV of pneumococcal carriage for MCPP was relatively high (Table). NPV for the CXR-AC outcomes were lower. Stratifying by prior antibiotic use and PCV status had little impact on findings. NPV decreases as prevalence of PP increases and specificity decreases (figure).

Figure: Negative predictive value of carriage as a function of specificity and prevalence of pneumococcal pneumonia among childhood cases of severe/very servere hospitalized

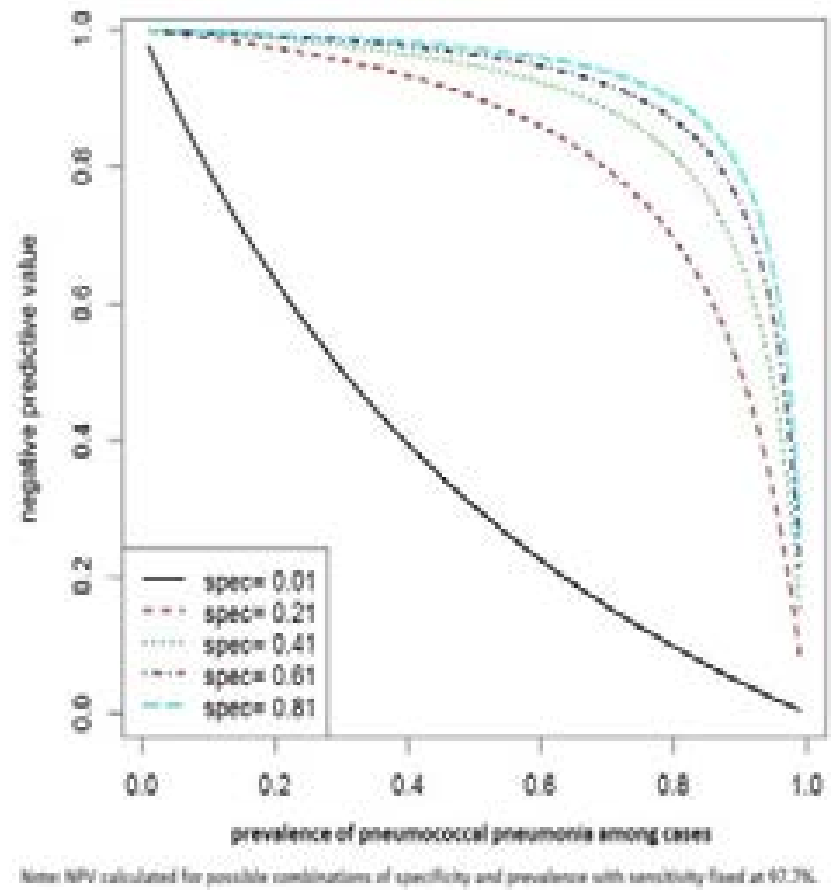


Conclusion: Absence of pneumococcal carriage might increase specificity of PP definitions which might be applicable in PCV impact evaluations. However, the lower NPVs for CXR-AC and for CXR-AC/other infiltrate plus elevated CRP need further clarification.

No conflict of interest

ISPPD-0180

Pneumococcal Colonization and Carriage

\section{PNEUMOCOCCAL SEROTYPES IN SWEDEN NOW AND THEN}

B. Trollfors ${ }^{1}$, S. Berg ${ }^{1}$, E. Backhaus ${ }^{2}$

${ }^{1}$ Pediatrics, Queen Silvia's Children's Hospital Sahlgrenska University, Gothenburg, Sweden; ${ }^{2}$ Pediatrics, Sahlgrenska University Hospital, Gothenburg, Sweden

Background and Aims: The serotype distribution of pneumococci has changed after large-scale use of pneumococcal conjugate vaccines (PCV). Data indicate that serotypes also changed over time before availability of PCV. The aim of this study was to compare serotypes of pneumococci obtained from normally sterile body fluids in 2 Swedish studies with strains isolated during the $1970 s+1980$ s and during $1998-2001$, i.e. long before any PCV was available.

Methods: 215 strains were serotyped in the old and 836 strains in the new study. Serotyping was performed with the capsular swelling test.

Results: The 7 most common serotypes in the first study were in descending order $3,4,14,7 \mathrm{~F}, 23 \mathrm{~F}, 6 \mathrm{~A}$ and $6 \mathrm{~B}$ and in the new study $1,7 F, 9 \mathrm{~V}, 14,4,12 \mathrm{~F}$ and 6B. Significant increases were seen in serotypes $1(2-14 \%), 9 \mathrm{~V}(2-9 \%)$ and $12 \mathrm{~F}(<1-6 \%)$. Serotype 3 decreased significantly $(12-4 \%)$. Serotype 19A, which has increased significantly in the US after widespread use of PCV7 and in 2 countries before PCV7 was introduced, (Israel, Korea), remained unchanged in the present comparison (3.7 and $3.1 \%$ ).

Conclusions: Major changes in the serotype distribution occurred in Sweden (as in many other countries) before any PCV was available. Changes in serotype distribution may therefore in some cases be due to natural changes and not only to replacement of vaccine types with nonvaccine types.

No conflict of interest

ISPPD-0489

Pneumococcal Colonization and Carriage

\section{NASOPHARYNGEAL CARRIAGE OF STREPTOCOCCUS PNEUMONIAE AMONG CHILDREN IN INDIA AND NEIGHBORING COUNTRIES}

B. Wahl ${ }^{1}$, M. Knoll ${ }^{1}$, A. Sutherland ${ }^{1}$, K. O'Brien ${ }^{1}$

${ }^{1}$ International Vaccine Access Center, Johns Hopkins Bloomberg School of Public Health, Baltimore MD, USA

Background and Aims: Streptococcus pneumoniae readily colonizes the nasopharynx (NP) of healthy children. Studies have demonstrated that carriage is a necessary precondition for invasive pneumococcal disease (IPD). We assessed pneumococcal NP colonization prevalence in India and in neighboring countries to assess if there were systematic differences.

Methods: We reviewed published and unpublished studies reporting on pneumococcal colonization prevalence among healthy children <15 years of age in India and neighboring countries from 1993 to 2013 . Study characteristics, colonization prevalence, and antibiotic resistance were systematically abstracted. We compared agespecific colonization prevalence in India to other countries.

Results: We identified 15 pneumococcal NP colonization studies from India and 21 from surrounding countries. In all studies, colonization prevalence increased immediately following birth and peaked between 5-9 months of age; peak prevalence for studies in India (range: 46-77\%) was similar to other countries (42-85\%). Colonization prevalence did not decline markedly $(>30 \%)$ in any studies during age 1-10 years, except one India study with a rapid decrease after age 7 months. Pneumococcal NP colonization prevalence after age 1 year in India (range $20-57 \%$ ) overlapped with prevalence in other countries (28-83\%). Data were insufficient to determine whether antimicrobial resistance or antibiotic use explained variation in colonization between studies.

Conclusions: Pediatric pneumococcal NP colonization prevalence in India is similar to surrounding countries. Risk factor prevalence for NP colonization was not reported and may explain colonization variation between studies. Pneumococcal colonization prevalence varies between studies but does not systematically vary between countries in South and Southeast Asia.

Conflict of interest 
ISPPD-0412

Pneumococcal Colonization and Carriage

\section{CARRIAGE OF STREPTOCOCCUS PNEUMONIAE IN 24-MONTH-OLD CHILDREN DETECTED USING MOLECULAR METHODS}

A.L. Wyllie ${ }^{1}$, J. van Engelsdorp Gastelaars ${ }^{1}$, M.L.J.N. Chu ${ }^{1}$, N.Y. Rots ${ }^{2}$, A.J. Wijmenga-Monsuur ${ }^{2}$, J.P. Bruin $^{3}$,
D. Bogaert ${ }^{1}$, K. Trzcinski ${ }^{1}$, E.A.M. Sanders ${ }^{1}$
${ }^{1}$ Pediatric Immunology and Infectious Diseases, Wilhelmina Children's Hospital University Medical Center Utrecht, Utrecht, Netherlands; ${ }^{2}$ Cen-
tre for Immunology of Infectious Diseases and Vaccines, National Institute for Public Health and the Environment (RIVM), Bilthoven, Netherlands;
${ }^{3}$ Diagnostic Microbiology, Regional Laboratory of Public Health, Haarlem, Netherlands

Background and Aims: The gold standard for Streptococcus pneumoniae carriage detection is the conventional culture of a nasopharyngeal swab. Saliva however, has a history of being one of the most sensitive methods in surveillances on pneumococcal colonisation (Heffron, 1939). Here, we compared the sensitivity of nasopharyngeal swabs and saliva samples from PCV7-vaccinated 24-month-old children tested for S. pneumoniae and nasopharyngeal serotype carriage using conventional and molecular diagnostic methods.

Methods: Nasopharyngeal and saliva samples were simultaneously collected from 289 asymptomatic 24-month-old children, cultured and pneumococcal strains were serotyped by Quellung method. DNA extracted from harvests of all bacterial growth on pneumococcus-selective medium was tested for S. pneumoniae and serotypes present using quantitative-PCR (qPCR) targeting species-specific genes lytA (Carvalho, 2007) and piaA and sequences specific for subset of serotypes (Azzari, 2010).

Results: Altogether 240 (85\%) of 289 24-month-old children were identified as carriers by any method. Molecular detection of S. pneumoniae in culture-enriched nasopharyngeal samples had highest sensitivity (73\%) followed by qPCR of cultured saliva (60\%) and conventional culture of nasopharyngeal swabs $(57 \%)$. Isolation of live pneumococci from saliva generally failed due to abundant polymicrobial growth. For the subset of serotypes targeted by qPCR (1, 3, 6A/B/C/D, 7A/F, 8, 9A/V, 10A/B, 14, 15A/B/C/D, 19A, 20, 22AF, 23F, 33A/F and 38) the number qPCR-positive results increased $2.2 \times$ when compared to culture results for nasopharyngeal samples alone. Conclusion: Application of molecular methods to detect $S$. pneumoniae in culture-enriched samples and testing saliva along with nasopharyngeal swabs significantly increased the number of carriers and strains detected.

No conflict of interest

ISPPD-0239

Pneumococcal Colonization and Carriage

\section{CARRIAGE OF STREPTOCOCCUS PNEUMONIAE IN SALIVA OF DUTCH PRIMARY SCHOOL CHILDREN}

A.L. Wyllie ${ }^{1}$, M.H.B. Schellens ${ }^{1}$, M.L.J.N. Chu ${ }^{1}$, J. van Engelsdorp Gastelaars ${ }^{1}$, D. Bogaert ${ }^{1}$, E.A.M. Sanders ${ }^{1}$, K. Trzcin$\mathrm{ski}^{1}$

${ }^{1}$ Pediatric Immunology and Infectious Diseases, Wilhelmina Children's Hospital University Medical Center Utrecht, Utrecht, Netherlands

Background and Aims: Saliva has a history as being one of the most sensitive methods for Streptococcus pneumoniae carriage detection (Heffron, 1939). Here, we applied molecular diagnostic methods to study pneumococcal carriage in saliva from schoolchildren.

Methods: Saliva was collected from 50 students (aged 5 to 10 years) of a rural school near Utrecht, transported to the lab on wet ice, cultured and the remaining volume stored frozen. Cultures were inspected for S. pneumoniae colonies and then all bacterial growth was harvested. DNA extracted from raw and culture-enriched samples was tested for the presence of S. pneumoniae specific genes lytA and piaA using quantitative-PCR (qPCR) and considered positive when both genes had $C_{T}$ values below 40 . Sample serotype composition was determined in DNA extracted from culture-enriched saliva samples using qPCR (Azzari, 2010) and by analysing sequences generated by conventional PCR (Carvalho, 2010; Carvalho 2013).

Results: Two children (4\%) were culture-positive for S. pneumoniae. Thirty-two (64\%) children were qPCR-positive for S. pneumoniae in raw saliva and $44(88 \%)$ in culture-enriched samples. Using molecular methods to determine sample serotype composition, we detected 83 pneumococcal strains of 22 serotypes in 40 of 50 samples from carriers, with 26 carriers ( $59 \%$ of 44 ) positive for 2 to 6 serotypes.

Conclusion: Conventional culture detection of S. pneumoniae in saliva is extremely difficult due to saliva's polymicrobial nature. These limitations were addressed by combining culture-enrichment and sensitive molecular methods, resulting in more than ten-fold higher rates of pneumococcal carriage and high rates of co-colonisation detected in schoolchildren.

No conflict of interest 